\title{
Comparative analysis of human and bovine protein kinases reveals unique relationship and functional diversity
}

\author{
Nuzhat N. Kabir ${ }^{1}$ and Julhash U. Kazi ${ }^{1,2 *}$ \\ ${ }^{1}$ Laboratory of Computational Biochemistry, KN Biomedical Research Institute, Bagura Road, \\ Barisal, Bangladesh. \\ ${ }^{2}$ Quality Control Section, Opsonin Pharma Limited, Bagura Road, Barisal, Bangladesh.
}

\begin{abstract}
Reversible protein phosphorylation by protein kinases and phosphatases is a common event in various cellular processes. The eukaryotic protein kinase superfamily, which is one of the largest superfamilies of eukaryotic proteins, plays several roles in cell signaling and diseases. We identified 482 eukaryotic protein kinases and 39 atypical protein kinases in the bovine genome, by searching publicly accessible genetic-sequence databases. Bovines have 512 putative protein kinases, each orthologous to a human kinase. Whereas orthologous kinase pairs are, on an average, $90.6 \%$ identical, orthologous kinase catalytic domain pairs are, on an average, $95.9 \%$ identical at the amino acid level. This bioinformatic study of bovine protein kinases provides a suitable framework for further characterization of their functional and structural properties.
\end{abstract}

Key words: ePK, kinome, aPK, bovine.

Received: September 25, 2010; Accepted: June 1, 2011.

The protein kinase family is one of the largest families of proteins. Protein kinases play important roles in many intracellular or intercellular signaling pathways, resulting in cell proliferation, gene expression, metabolism, motility, membrane transport, apoptosis and differentiation. Furthermore, they modulate the activity of their substrate proteins by phosphorylating serine, threonine or tyrosine residues that mediate the activation, inhibition, translocation or degradation of substrate proteins (Brognard and Hunter, 2011).

Protein kinases are subdivided into two distinct superfamilies, referred to as eukaryotic protein kinases (ePKs) and atypical protein kinases (aPKs) (Hanks and Hunter, 1995). ePKs contain a conserved catalytic domain of approximately 250 amino acids. This domain is divided into 12 subdomains with highly conserved individual amino acids and motifs (Hanks et al., 1988). Within this domain, three motifs, 'VAIK', 'HRD' and 'DFG', are critical for the catalytic function, even though any residue from this region is fully conserved in all family members (Manning et al., 2002b). Conservation of these typical motifs is thought to be due to selection pressure for conserving important functions, such as the interaction with ATP, and the transfer of a phosphate group to the substrate. aPKs are functionally known to have kinase activity which lack significant sequence similarity to the ePK domain.

Send correspondence to Julhash U. Kazi. Quality Control Section, Opsonin Pharma Limited, Bagura Road, Barisal-8200, Bangladesh. E-mail. Icb.kazi@gmail.com.
The sequencing of several vertebrate genomes has been completed. Initial estimates of the number of protein kinases in the human genome place this at around 1000 (Hunter, 1987), with later studies identifying 518 putative protein kinases (Manning et al., 2002b). The mouse and rat genomes contain 540 and 555 protein kinases, respectively, with 509 human orthologs (Caenepeel et al., 2004; Kazi et al., 2008), thereby implying possible functional conservation across species. In the present study, and by using sensitive bioinformatics approaches we identified the near complete set of bovine protein kinases. These were further classified into groups, families and subfamilies, based on the Hanks et al. (1988) and Manning et al. (2002b) classification scheme. This classification reveals many kinases that are conserved between bovine and human, thus reflecting functional constraints of these protein kinases in the core of signaling pathway. This study provides a suitable framework for further characterization of the functional and structural properties of these protein kinases.

A search was made of bovine proteome sequences available in GenBank (Benson et al., 2010) and Ensembl (Hubbard et al., 2009) for bovine protein kinases, using various tools. A preliminary search for protein kinases was performed using PSI-BLAST (Altschul et al., 1997), against the bovine proteome with an e-value threshold of 0.0001 , and an h-value of 0.1 for five iterations. Previously published human (Manning et al., 2002b), mouse (Caenepeel et al., 2004) and rat (Kazi et al., 2008) eukaryotic protein kinases and kinase catalytic domains, as well as 
eukaryotic protein kinase catalytic domains from a variety of organisms available at the kinase.com database were used as query sequences. A further search for protein kinases was performed using HMMER (Eddy, 1998). A Hidden Markov profile was created and validated by means of known eukaryotic protein-kinase catalytic domains. A further search was made for atypical protein kinases, using human, mouse and rat atypical protein kinases by PSI-BLAST or HMMER. Hits identified by using the different methods were combined, and duplicate records removed. Where splice variants were encountered, the variant showing either the closest proximity to the human ortholog, or the longest protein encoding variant, was recorded. All protein kinases were then evaluated for the presence of a conserved eukaryotic protein kinase domain (Hanks et al., 1988; Manning et al., 2002b). Catalytic domains were defined using RPS-BLAST in the BLAST package (Altschul et al., 1997) against the Pfam database (Finn et al., 2010), and sequence alignments carried out with AlinX implemented in the Vector-NTI package (Lu and Moriyama, 2004). Alignments were then manually edited, and all the kinases manually evaluated. Finally, 482 eukaryotic protein kinases and 39 atypical protein kinases were identified (Table S1). The primary names of the protein kinases were derived from their respective homologs in human (Manning et al., 2002b), mouse (Caenepeel et al., 2004) and rat (Kazi et al., 2008) protein kinases. On deriving a second name and synonyms from the Entrez Gene records (Maglott et al., 2005), the full protein names were retrieved thence. Representative records in Entrez Gene, corresponding to each bovine sequence, were identified, whereupon related information was included. Based upon the human protein kinases classification scheme (Manning et al., 2002b), these protein kinases were further classified into 10 groups, 129 families and 81 subfamilies (Table 1 and Table S1).

Previous studies have shown that almost all human protein-kinase orthologs are present in mouse and rat genomes (Caenepeel et al., 2004; Kazi et al., 2008). Thus, a search among bovine kinase sequences was conducted for the orthologous kinases using BLASTP (Altschul et al., 1997). The results were parsed, the symmetrically best hits being considered as orthologous kinases. The orthology re- lationships were further analyzed by CLUSTALW alignment (Thompson et al., 1997), followed by phylogenetic analysis. The latter was carried out by phylogenetic tree option incorporated into the CLUSTALW program. The NJ clustering algorithm was used for drawing bootstrap trees. As almost all bovine and human protein kinases exist as orthologous pairs (Figure S1), the similar functions in both organisms give to understand their evolutionary conservation. Human and bovine genomes contain 512 common protein kinase orthologs. Our search could not identify seven human protein kinase orthologs, likely due to the incomplete nature of bovine genome sequencing data (Table 2). In these seven human protein kinases, only TAF1L is absent in the chimpanzee genome. All are present in various higher eukaryotes, such as of orangutans and monkeys. Ten protein kinases were absent in the human genome, eight of which being bovine specific and the other two, PLK5 and TSSK5 found in other genomes (Table 2). EphB1L might be a retrotransposed copy of the EphB1 gene, with $89.2 \%$ of amino acid sequence identity with the EphB1 protein.

Several proteins, such as ErbB3, SCYL1 and KSR1, have an inactive catalytic domain (Citri et al., 2003; Manning et al., 2002a). These inactive kinases, besides acting mainly as adaptor proteins, or dimerizing with active kinases, have also been shown to be involved in various cellular functions (Salerno et al., 2005; Schmidt et al., 2007; Sergina et al., 2007). Three conserved motifs, 'VAIK', 'HRD' and 'DFG', are important for catalytic activities. Inactive kinases lack at least one of these three conserved motifs. Fifty catalytic domains and 45 protein kinases in the human genome were predicted as catalytically inactive due to the lack of at least one of the three conserved residues (Manning et al., 2002b). The bovine complement of inactive kinases is equivalent to that of the human (Table S1).

All the human and bovine orthologous protein kinase pairs and orthologous catalytic domain pairs were analyzed for the percentage of identity by AlignX incorporated into Vector-NTI (Lu and Moriyama, 2004). In protein sequence alignments of orthologous kinase pairs we observed a wide variation in local sequence conservation (Figure 1A). These were, on an average, $90.6 \%$ identical (amino acid se-

Table 1 - Protein-kinases and their distribution by groups in different genomes.

\begin{tabular}{lccccccccccc}
\hline Species & AGC & CAMK & CK1 & CMGC & Other & RGC & STE & TK & TKL & Atypical & Total \\
\hline Human & 63 & 74 & 12 & 61 & 83 & 5 & 47 & 90 & 43 & 40 & 518 \\
Bovine & 62 & 75 & 12 & 61 & 85 & 5 & 47 & 91 & 44 & 39 & 521 \\
Rat & 60 & 112 & 11 & 61 & 84 & 7 & 46 & 91 & 43 & 40 & 555 \\
Mouse & 60 & 97 & 11 & 60 & 84 & 7 & 47 & 90 & 43 & 39 & 538 \\
Drosophila melanogaster & 30 & 32 & 10 & 33 & 45 & 6 & 18 & 32 & 17 & 17 & 240 \\
Caenorhabditis elegans & 30 & 46 & 85 & 49 & 67 & 27 & 25 & 90 & 15 & 20 & 454 \\
Saccharomyces cerevisiae & 17 & 21 & 4 & 21 & 38 & 0 & 14 & 0 & 0 & 15 & 130 \\
\hline
\end{tabular}


Table 2 - Lineage-specific protein kinases.

\begin{tabular}{lcccl}
\hline Found in & Gene Name & Introns & Paralog & Other orthologs \\
\hline Human and bovine & & \multicolumn{2}{c}{512 genes } \\
\hline Human & CK1a2 & No & CK1a1 (91\%) & Chimpanzee, Orangutan, Monkey \\
& TAF1L & No & TAF1 $(94 \%)$ & Orangutan, Monkey \\
& PKACg & No & PKACa $(83 \%)$ & Chimpanzee, Orangutan \\
& LTK & Yes & - & Chimpanzee, Orangutan, Rat, Mouse, Horse, Chicken \\
& ROS & Yes & - & \\
& PSKH2 & Yes & - & Chimpanzee, Monkey, Dog, Orangutan, Rabbit, Horse \\
& PRKY & Yes & PRKX (92\%) & Chimpanzee, Gorilla, Monkey \\
\hline Bovine & AURL & Yes & AurB $(61 \%)$ & - \\
& CK1b & Yes & CK1a $(81 \%)$ & - \\
& EphB1L & No & EphB1 $(89.2 \%)$ & - \\
FERL & Yes & FER (82\%) & - \\
MARKL & Yes & - & - \\
MASTL2 & Yes & - & - \\
Par-1L & Yes & - & - \\
PLK5 & Yes & - & Orangutan, Monkey, Mouse, Rat \\
RYKL & Yes & - & - \\
TSSK5 & Yes & - & Dog, Horse, Mouse, Rat \\
\hline
\end{tabular}

In 'Paralog' parentheses show \% of identity (amino acid) with respective paralog.
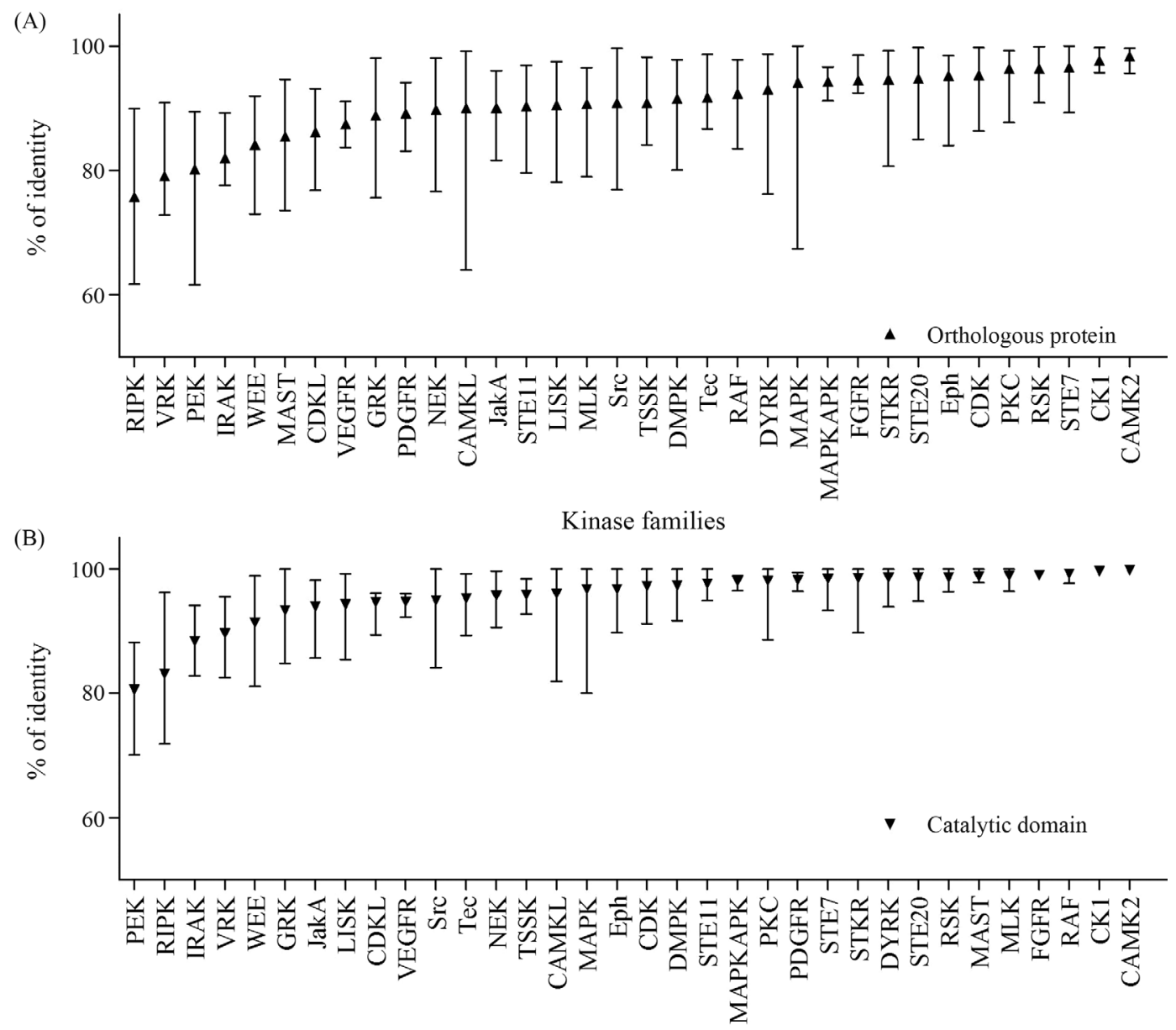

Kinase families

Figure 1 - Conservation within orthologous kinases (A) and catalytic domains (B) is family-dependent. Triangles indicate mean identity within selected families, and bars indicate the range. 
(A)

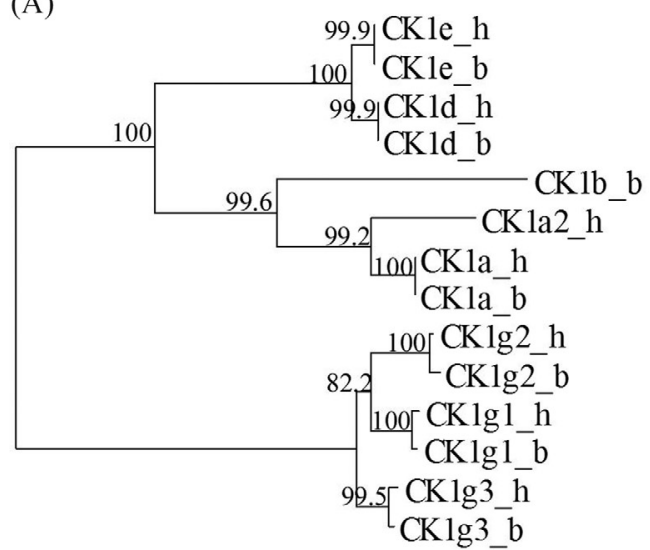

(B)

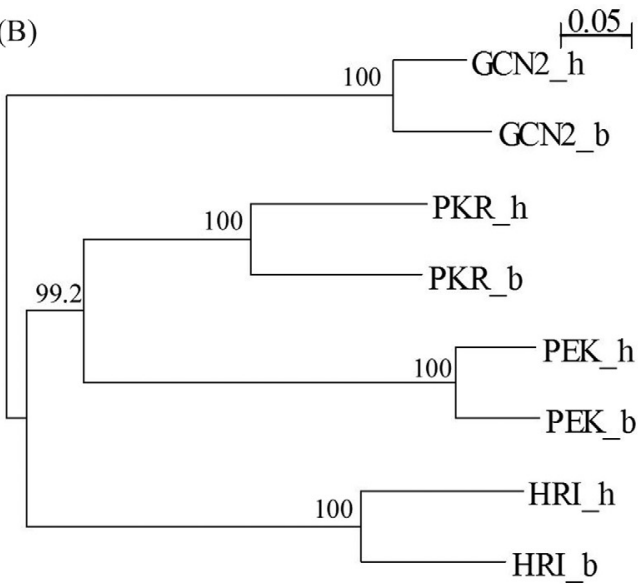

Figure 2 - Catalytic domain conservation is family-dependent. (A) The CK1 family is highly conserved, with zero to two amino acid changes between bovine and human orthologs. (B) Catalytic domains of the PEK family are poorly conserved between human and bovine. h, Human; b, Bovine.

quence), although some were as low as $47.7 \%$, with four pairs presenting high levels of sequence identity throughout the protein (Table S1). Although most differences between orthologs are due to amino acid substitution, many proteins contained substantial insertions or deletions (indels) between orthologs, which may account for many of the functional differences between species. Several proteins contained insertions or deletions (indels), as shown by sequence alignment (Table S1). These comparisons are also informative within the conserved domains. Although orthologous catalytic domains were, on an average, 95.9\% identical, some were as low as $63.8 \%$. Sixty two pairs were identical across the full domain, whereas 48 differed by only one amino acid (Table S1), thus indicative of strong conservative pressure throughout the catalytic domain. Catalytic domain pairs showed clearly family-dependent variability (Figure 1B). For example, of the six casein kinase 1 (CK1) family domain pairs, three were identical, and the other three differed by two residues, an average difference of only $0.4 \%$, thereby indicating that changes in almost any amino acid within the domain destroyed some function, and thus have been eliminated by evolution (Figure 2A). At the other extreme, PEK family catalytic domain pairs are $70-88 \%$ identical, thereby implying that the core functions of this family of kinases do not greatly constrain the domain sequence (Figure 2B).

Our study presents a bioinformatic overview and evolutionary insight into the kinases within the bovine genome. Comparison with the human kinome revealed the evolutionary conservation of the protein kinase function. The curated kinase dataset from the bovine genome, presented here, could serve as a framework for further investigation of this important gene family.

\section{Acknowledgments}

This work was supported by a KN Biomedical Research Grant. We are grateful to Nadid Ahnaf Kazi.

\section{References}

Altschul SF, Madden TL, Schaffer AA, Zhang J, Zhang Z, Miller W and Lipman DJ (1997) Gapped BLAST and PSI-BLAST: A new generation of protein database search programs. Nucleic Acids Res 25:3389-3402.

Benson DA, Karsch-Mizrachi I, Lipman DJ, Ostell J and Sayers EW (2010) GenBank. Nucleic Acids Res 38:D46-51.

Brognard J and Hunter T (2011) Protein kinase signaling networks in cancer. Curr Opin Genet Dev 21:4-11.

Caenepeel S, Charydczak G, Sudarsanam S, Hunter T and Manning G (2004) The mouse kinome: Discovery and comparative genomics of all mouse protein kinases. Proc Natl Acad Sci USA 101:11707-11712.

Citri A, Skaria KB and Yarden Y (2003) The deaf and the dumb: The biology of ErbB-2 and ErbB-3. Exp Cell Res 284:54-65.

Eddy SR (1998) Profile hidden Markov models. Bioinformatics 14:755-763.

Finn RD, Mistry J, Tate J, Coggill P, Heger A, Pollington JE, Gavin OL, Gunasekaran P, Ceric G, Forslund K, et al. (2010) The Pfam protein families database. Nucleic Acids Res 38:D211-222.

Hanks SK and Hunter T (1995) Protein kinases 6. The eukaryotic protein kinase superfamily: Kinase (catalytic) domain structure and classification. FASEB J 9:576-596.

Hanks SK, Quinn AM and Hunter T (1988) The protein kinase family: Conserved features and deduced phylogeny of the catalytic domains. Science 241:42-52.

Hubbard TJ, Aken BL, Ayling S, Ballester B, Beal K, Bragin E, Brent S, Chen Y, Clapham P, Clarke L, et al. (2009) Ensembl 2009. Nucleic Acids Res 37:D690-697.

Hunter T (1987) A thousand and one protein kinases. Cell 50:823-829.

Kazi JU, Kabir NN and Soh JW (2008) Bioinformatic prediction and analysis of eukaryotic protein kinases in the rat genome. Gene 410:147-153.

Lu G and Moriyama EN (2004) Vector NTI, a balanced all-in-one sequence analysis suite. Brief Bioinform 5:378-388.

Maglott D, Ostell J, Pruitt KD and Tatusova T (2005) Entrez gene: Gene-centered information at NCBI. Nucleic Acids Res 33:D54-58. 
Manning G, Plowman GD, Hunter T and Sudarsanam S (2002a) Evolution of protein kinase signaling from yeast to man. Trends Biochem Sci 27:514-520.

Manning G, Whyte DB, Martinez R, Hunter T and Sudarsanam S (2002b) The protein kinase complement of the human genome. Science 298:1912-1934.

Salerno M, Palmieri D, Bouadis A, Halverson D and Steeg PS (2005) Nm23-H1 metastasis suppressor expression level influences the binding properties, stability, and function of the kinase suppressor of Ras1 (KSR1) Erk scaffold in breast carcinoma cells. Mol Cell Biol 25:1379-1388.

Schmidt WM, Kraus C, Hoger H, Hochmeister S, Oberndorfer F, Branka M, Bingemann S, Lassmann H, Muller M, MacedoSouza LI, et al. (2007) Mutation in the Scyll gene encoding amino-terminal kinase-like protein causes a recessive form of spinocerebellar neurodegeneration. EMBO Rep 8:691697.

Sergina NV, Rausch M, Wang D, Blair J, Hann B, Shokat KM and Moasser MM (2007) Escape from HER-family tyrosine kinase inhibitor therapy by the kinase-inactive HER3. Nature 445:437-441.
Thompson JD, Gibson TJ, Plewniak F, Jeanmougin F and Higgins DG (1997) The CLUSTAL_X windows interface: Flexible strategies for multiple sequence alignment aided by quality analysis tools. Nucleic Acids Res 25:4876-4882.

\section{Internet Resources}

Kinase.com database, Genomics, evolution and function of protein kinases, http://kinase.com/kinbase/FastaFiles/.

\section{Supplementary Material}

The following online material is available for this article:

Table S1 - List of bovine kinases with sequences.

Figure S1 - Phylogenetic trees for human and bovine kinases.

This material is available as part of the online article from http:/www.scielo.br/gmb.

Associate Editor: Alexandre Rodrigues Caetano

License information: This is an open-access article distributed under the terms of the Creative Commons Attribution License, which permits unrestricted use, distribution, and reproduction in any medium, provided the original work is properly cited. 


\begin{tabular}{|c|c|c|c|c|c|c|}
\hline Line \# & Name & Symbol & Group & Family & Subfamily & GenelD \\
\hline 1 & AKT1 & AKT1 & AGC & AKT & & 280991 \\
\hline 2 & AKT2 & AKT2 & AGC & AKT & & 534923 \\
\hline 3 & AKT3 & AKT3 & AGC & AKT & & 100137872 \\
\hline 4 & DMPK1 & & AGC & DMPK & GEK & \\
\hline 5 & DMPK2 & CDC42BPG & AGC & DMPK & GEK & 518121 \\
\hline 6 & MRCKa & CDC42BPA & AGC & DMPK & GEK & 538392 \\
\hline 7 & MRCKb & & AGC & DMPK & GEK & \\
\hline 8 & ROCK1 & ROCK1 & $A G C$ & DMPK & ROCK & 785911 \\
\hline 9 & ROCK2 & ROCK2 & AGC & DMPK & ROCK & 282041 \\
\hline 10 & CRIK & & $A G C$ & DMPK & & \\
\hline 11 & BARK1 & ADRBK1 & AGC & GRK & BARK & 282682 \\
\hline 12 & BARK2 & ADRBK2 & AGC & GRK & BARK & 282136 \\
\hline 13 & GPRK4 & GRK4 & $A G C$ & GRK & GRK & 512489 \\
\hline 14 & GPRK5 & GRK5 & AGC & GRK & GRK & 281801 \\
\hline 15 & GPRK6 & & $A G C$ & GRK & GRK & \\
\hline 16 & GPRK7 & GRK7 & AGC & GRK & GRK & 281802 \\
\hline 17 & RHOK & GRK1 & $A G C$ & GRK & GRK & 281457 \\
\hline 18 & MAST1 & MAST1 & AGC & MAST & & 539825 \\
\hline 19 & MAST2 & & $A G C$ & MAST & & \\
\hline 20 & MAST3 & MAST3 & AGC & MAST & & 534230 \\
\hline 21 & MAST4 & & AGC & MAST & & \\
\hline 22 & MASTL & MASTL & AGC & MASTL & & 540206 \\
\hline 23 & MASTL2 & & $A G C$ & MASTL & & \\
\hline 24 & LATS1 & LATS1 & AGC & NDR & & 535935 \\
\hline 25 & LATS2 & LATS2 & AGC & NDR & & 508208 \\
\hline 26 & NDR1 & STK38 & $A G C$ & NDR & & 533677 \\
\hline 27 & NDR2 & STK38L & $A G C$ & NDR & & 514787 \\
\hline 28 & PKACa & PRKACA & AGC & PKA & & 282322 \\
\hline 29 & PKACb & PRKACB & $A G C$ & PKA & & 282323 \\
\hline 30 & PRKX & PRKX & $A G C$ & PKA & & 505773 \\
\hline 31 & PDK1 & PDK1 & $A G C$ & PKB & & 100138102 \\
\hline 32 & PKCi & PRKCl & AGC & PKC & Atypical & 528478 \\
\hline 33 & $\mathrm{PKCz}$ & PRKCZ & AGC & PKC & Atypical & 286877 \\
\hline 34 & PKCa & PRKCA & AGC & PKC & Classical & 282001 \\
\hline 35 & $\mathrm{PKCb}$ & PRKCB1 & $A G C$ & PKC & Classical & 282325 \\
\hline 36 & $\mathrm{PKCg}$ & PRKCG & $A G C$ & PKC & Classical & 282002 \\
\hline 37 & PKCd & PRKCD & $A G C$ & PKC & Nobel & 505708 \\
\hline 38 & PKCe & PRKCE & AGC & PKC & Nobel & 507041 \\
\hline 39 & PKCh & PRKCH & $A G C$ & PKC & Nobel & 518542 \\
\hline 40 & PKCt & PRKCQ & AGC & PKC & Nobel & 505901 \\
\hline 41 & PKG1 & PRKG1 & AGC & PKG & & 282004 \\
\hline 42 & PKG2 & PRKG2 & $A G C$ & PKG & & 533330 \\
\hline 43 & PKN1 & PKN1 & $A G C$ & PKN & & 509080 \\
\hline 44 & PKN2 & PKN2 & AGC & PKN & & 519754 \\
\hline 45 & PKN3 & PKN3 & $A G C$ & PKN & & 505353 \\
\hline 46 & MSK1 & RPS6KA5 & $A G C$ & RSK & MSK & 504408 \\
\hline 47 & MSK2 & & $A G C$ & RSK & MSK & 523746 \\
\hline 48 & p70S6K & RPS6KB1 & $A G C$ & RSK & p70 & 404181 \\
\hline 49 & p70S6Kb & RPS6KB2 & $A G C$ & RSK & p70 & 506083 \\
\hline
\end{tabular}




\begin{tabular}{|c|c|c|c|c|c|c|}
\hline 50 & RSK1 & RPS6KA2 & AGC & RSK & RSK & 517953 \\
\hline 51 & RSK2 & RPS6KA3 & AGC & RSK & RSK & 511190 \\
\hline 52 & RSK3 & RPS6KA1 & AGC & RSK & RSK & 533908 \\
\hline 53 & RSK4 & RPS6KA6 & AGC & RSK & RSK & 526227 \\
\hline 54 & RSKL1 & RPS6KC1 & AGC & RSKL & & 533192 \\
\hline 55 & RSKL2 & RPS6KL1 & AGC & RSKL & & 530047 \\
\hline 56 & SgK494 & SGK494 & AGC & RSKL & & 527796 \\
\hline 57 & SGK & SGK1 & $A G C$ & SGK & & 515854 \\
\hline 58 & SGK2 & SGK2 & $A G C$ & SGK & & 517909 \\
\hline 59 & SGK3 & SGK3 & AGC & SGK & & 504480 \\
\hline 60 & YANK1 & STK32A & AGC & YANK & & 100125774 \\
\hline 61 & YANK2 & STK32B & AGC & YANK & & 529134 \\
\hline 62 & YANK3 & STK32C & AGC & YANK & & 524218 \\
\hline 63 & A6 & TWF1 & Atypical & A6 & & 506683 \\
\hline 64 & $A 6 r$ & TWF2 & Atypical & A6 & & 282024 \\
\hline 65 & ADCK3 & CABC1 & Atypical & $A B C 1$ & $A B C 1-A$ & 536925 \\
\hline 66 & ADCK4 & ADCK4 & Atypical & $A B C 1$ & $A B C 1-A$ & 514322 \\
\hline 67 & ADCK1 & ADCK1 & Atypical & $A B C 1$ & ABC1-B & 533372 \\
\hline 68 & ADCK5 & ADCK5 & Atypical & $A B C 1$ & ABC1-B & 522614 \\
\hline 69 & ADCK2 & ADCK2 & Atypical & ABC1 & ABC1-C & 539345 \\
\hline 70 & ChaK1 & TRPM7 & Atypical & Alpha & ChaK & 514642 \\
\hline 71 & ChaK2 & TRPM6 & Atypical & Alpha & ChaK & 527597 \\
\hline 72 & eEF2K & EEF2K & Atypical & Alpha & eEF2K & 521730 \\
\hline 73 & AlphaK1 & ALPK3 & Atypical & Alpha & & \\
\hline 74 & AlphaK2 & ALPK2 & Atypical & Alpha & & 510218 \\
\hline 75 & AlphaK3 & ALPK1 & Atypical & Alpha & & 524375 \\
\hline 76 & $\mathrm{BCR}$ & $\mathrm{BCR}$ & Atypical & $\mathrm{BCR}$ & & 789892 \\
\hline 77 & BRD2 & BRD2 & Atypical & BRD & & 505358 \\
\hline 78 & BRD3 & BRD3 & Atypical & BRD & & 525051 \\
\hline 79 & BRD4 & BRD4 & Atypical & BRD & & 614048 \\
\hline 80 & BRDT & BRDT & Atypical & BRD & & 523971 \\
\hline 81 & FASTK & FASTK & Atypical & FAST & & 509781 \\
\hline 82 & G11 & STK19 & Atypical & G11 & & 508320 \\
\hline 83 & $\mathrm{H} 11$ & HSPB8 & Atypical & $\mathrm{H} 11$ & & 539524 \\
\hline 84 & BCKDK & BCKDK & Atypical & PDHK & & 505005 \\
\hline 85 & PDHK1 & PDK1 & Atypical & PDHK & & 528655 \\
\hline 86 & PDHK2 & PDK2 & Atypical & PDHK & & 524075 \\
\hline 87 & PDHK3 & PDK3 & Atypical & PDHK & & 510841 \\
\hline 88 & PDHK4 & PDK4 & Atypical & PDHK & & 507367 \\
\hline 89 & ATM & ATM & Atypical & PIKK & ATM & 526824 \\
\hline 90 & ATR & ATR & Atypical & PIKK & ATR & 504869 \\
\hline 91 & DNAPK & PRKDC & Atypical & PIKK & DNAPK & 512740 \\
\hline 92 & FRAP & MTOR & Atypical & PIKK & FRAP & 100139219 \\
\hline 93 & SMG1 & SMG1 & Atypical & PIKK & SMG1 & 525143 \\
\hline 94 & TRRAP & TRRAP & Atypical & PIKK & TRRAP & 507169 \\
\hline 95 & RIOK1 & RIOK1 & Atypical & $\mathrm{RIO}$ & $\mathrm{RIO1}$ & 516289 \\
\hline 96 & RIOK2 & RIOK2 & Atypical & RIO & $\mathrm{RIO2}$ & 540772 \\
\hline 97 & RIOK3 & RIOK3 & Atypical & RIO & $\mathrm{RIO3}$ & 522917 \\
\hline 98 & TAF1 & TAF1 & Atypical & TAF1 & & 504792 \\
\hline 99 & TIF1a & TRIM33 & Atypical & TIF1 & & 533296 \\
\hline
\end{tabular}




\begin{tabular}{|c|c|c|c|c|c|c|}
\hline 100 & TIF1b & & Atypical & TIF1 & & \\
\hline 101 & TIF1g & TRIM24 & Atypical & TIF1 & & 537246 \\
\hline 102 & CaMK1a & CAMK1 & CAMK & CAMK1 & & 520498 \\
\hline 103 & CaMK1b & PNCK & CAMK & CAMK1 & & 614251 \\
\hline 104 & CaMK1d & CAMK1D & CAMK & CAMK1 & & 526873 \\
\hline 105 & CaMK1g & CAMK1G & CAMK & CAMK1 & & 511504 \\
\hline 106 & CaMK2a & CAMK2A & CAMK & CAMK2 & & 530719 \\
\hline 107 & CaMK2b & CAMK2B & CAMK & CAMK2 & & 525416 \\
\hline 108 & CaMK2d & CAMK2D & CAMK & CAMK2 & & 532713 \\
\hline 109 & CaMK2g & CAMK2G & CAMK & CAMK2 & & 282162 \\
\hline 110 & CaMK4 & oc10033708 & CAMK & CAMK4 & & 100337083 \\
\hline 111 & AMPKa1 & PRKAA1 & CAMK & CAMKL & AMPK & 540404 \\
\hline 112 & AMPKa2 & PRKAA2 & CAMK & CAMKL & AMPK & 538954 \\
\hline 113 & BRSK1 & BRSK1 & CAMK & CAMKL & BRSK & 538009 \\
\hline 114 & BRSK2 & BRSK2 & CAMK & CAMKL & BRSK & 535868 \\
\hline 115 & CHK1 & CHEK1 & CAMK & CAMKL & CHK1 & 513678 \\
\hline 116 & HUNK & HUNK & CAMK & CAMKL & HUNK & 537640 \\
\hline 117 & LKB1 & STK11 & CAMK & CAMKL & LKB & 515245 \\
\hline 118 & MARK1 & MARK1 & CAMK & CAMKL & MARK & 539563 \\
\hline 119 & MARK2 & MARK2 & CAMK & CAMKL & MARK & 535197 \\
\hline 120 & MARK3 & MARK3 & CAMK & CAMKL & MARK & 534872 \\
\hline 121 & MARK4 & MARK4 & CAMK & CAMKL & MARK & 525675 \\
\hline 122 & MARKL & LOC618482 & CAMK & CAMKL & MARK & 618482 \\
\hline 123 & MELK & MELK & CAMK & CAMKL & MELK & 520088 \\
\hline 124 & NIM1 & LOC530210 & CAMK & CAMKL & NIM1 & 530210 \\
\hline 125 & NuaK1 & NUAK1 & CAMK & CAMKL & NuaK & 519892 \\
\hline 126 & NuaK2 & NUAK2 & CAMK & CAMKL & NuaK & 518632 \\
\hline 127 & PASK & PASK & CAMK & CAMKL & PASK & 512154 \\
\hline 128 & QIK & SNF1LK2 & CAMK & CAMKL & QIK & 539570 \\
\hline 129 & QSK & SIK3 & CAMK & CAMKL & QIK & 506539 \\
\hline 130 & SIK & SIK & CAMK & CAMKL & QIK & \\
\hline 131 & SNRK & SNRK & CAMK & CAMKL & SNRK & 536909 \\
\hline 132 & SgK495 & STK40 & CAMK & CAMK-Unique & & 515723 \\
\hline 133 & STK33 & STK33 & CAMK & CAMK-Unique & & 528309 \\
\hline 134 & VACAMKL & CAMKV & CAMK & CAMK-Unique & & 525741 \\
\hline 135 & CASK & CASK & CAMK & CASK & & 404149 \\
\hline 136 & DAPK1 & DAPK1 & CAMK & DAPK & & 540873 \\
\hline 137 & DAPK2 & DAPK2 & CAMK & DAPK & & 529131 \\
\hline 138 & DAPK3 & DAPK3 & CAMK & DAPK & & 525506 \\
\hline 139 & DRAK1 & STK17A & CAMK & DAPK & & 513665 \\
\hline 140 & DRAK2 & STK17B & CAMK & DAPK & & 511373 \\
\hline 141 & DCAMKL1 & & CAMK & DCAMKL & & \\
\hline 142 & DCAMKL2 & DCLK2 & CAMK & DCAMKL & & 536110 \\
\hline 143 & DCAMKL3 & DCLK3 & CAMK & DCAMKL & & 507205 \\
\hline 144 & MAPKAPK2 & MAPKAPK2 & CAMK & MAPKAPK & MAPKAPK & 788091 \\
\hline 145 & MAPKAPK3 & MAPKAPK3 & CAMK & MAPKAPK & MAPKAPK & 615215 \\
\hline 146 & MAPKAPK5 & MAPKAPK5 & CAMK & MAPKAPK & MAPKAPK & 535625 \\
\hline 147 & MNK1 & MKNK1 & CAMK & MAPKAPK & MNK & 525647 \\
\hline 148 & MNK2 & MKNK2 & CAMK & MAPKAPK & MNK & 538519 \\
\hline 149 & caMLCK & MYLK3 & CAMK & MLCK & & 526379 \\
\hline
\end{tabular}




\begin{tabular}{|c|c|c|c|c|c|c|}
\hline 150 & SgK085 & MYLK4 & CAMK & MLCK & & 518025 \\
\hline 151 & skMLCK & MYLK2 & CAMK & MLCK & & 533378 \\
\hline 152 & smMLCK & MYLK & CAMK & MLCK & & 338037 \\
\hline 153 & TTN & TTN & CAMK & MLCK & & 540561 \\
\hline 154 & PHKg1 & PHKG1 & CAMK & PHK & & 540682 \\
\hline 155 & PHKg2 & PHKG2 & CAMK & PHK & & 512670 \\
\hline 156 & PIM1 & PIM1 & CAMK & PIM & & 281402 \\
\hline 157 & PIM2 & PIM2 & CAMK & PIM & & 508424 \\
\hline 158 & PIM3 & oc10033665 & CAMK & PIM & & 100336651 \\
\hline 159 & PKD1 & PRKD1 & CAMK & PKD & & 533270 \\
\hline 160 & PKD2 & PRKD2 & CAMK & PKD & & 782793 \\
\hline 161 & PKD3 & PRKD3 & CAMK & PKD & & 538447 \\
\hline 162 & PSKH1 & PSKH1 & CAMK & PSK & & 509656 \\
\hline 163 & CHK2 & CHEK2 & CAMK & RAD53 & & 518897 \\
\hline 164 & Trb1 & TRIB1 & CAMK & Trbl & & 521857 \\
\hline 165 & Trb2 & TRIB2 & CAMK & Trbl & & 352960 \\
\hline 166 & Trb3 & TRIB3 & CAMK & Trbl & & 538465 \\
\hline 167 & Obscn & OBSCN & CAMK & Trio & & 508247 \\
\hline 168 & SPEG & SPEG & CAMK & Trio & & 523490 \\
\hline 169 & Trad & KALRN & CAMK & Trio & & 540675 \\
\hline 170 & Trio & TRIO & CAMK & Trio & & 538292 \\
\hline 171 & SSTK & TSSK6 & CAMK & TSSK & & 539707 \\
\hline 172 & TSSK1 & TSSK1B & CAMK & TSSK & & 529193 \\
\hline 173 & TSSK2 & TSSK2 & CAMK & TSSK & & \\
\hline 174 & TSSK3 & TSSK3 & CAMK & TSSK & & 538659 \\
\hline 175 & TSSK4 & TSSK4 & CAMK & TSSK & & 539477 \\
\hline 176 & TSSK5 & LOC523023 & CAMK & TSSK & & 523023 \\
\hline 177 & CK1a & CSNK1A1 & CK1 & CK1 & & 282684 \\
\hline 178 & CK1b & CSNK1B & CK1 & CK1 & & 785423 \\
\hline 179 & CK1d & CSNK1D & CK1 & CK1 & & 523542 \\
\hline 180 & CK1e & CSNK1E & CK1 & CK1 & & 768234 \\
\hline 181 & CK1g1 & CSNK1G1 & CK1 & CK1 & & 527889 \\
\hline 182 & CK1g2 & CSNK1G2 & CK1 & CK1 & & 506060 \\
\hline 183 & CK1g3 & & CK1 & CK1 & & \\
\hline 184 & TTBK1 & & CK1 & TTBK & & \\
\hline 185 & TTBK2 & TTBK2 & CK1 & TTBK & & 541215 \\
\hline 186 & VRK1 & VRK1 & CK1 & VRK & & 618880 \\
\hline 187 & VRK2 & VRK2 & CK1 & VRK & & 523902 \\
\hline 188 & VRK3 & VRK3 & CK1 & VRK & & 520302 \\
\hline 189 & CDC2 & $\mathrm{CDC2}$ & CMGC & CDK & CDC2 & 281061 \\
\hline 190 & CDK2 & CDK2 & CMGC & CDK & CDC2 & 519217 \\
\hline 191 & CDK3 & CDK3 & CMGC & CDK & $\mathrm{CDC2}$ & 618631 \\
\hline 192 & CDK10 & CDK10 & CMGC & CDK & CDK10 & 615171 \\
\hline 193 & CDK4 & CDK4 & CMGC & CDK & CDK4 & 510618 \\
\hline 194 & CDK6 & CDK6 & CMGC & CDK & CDK4 & 511754 \\
\hline 195 & CDK5 & CDK5 & CMGC & CDK & CDK5 & 281066 \\
\hline 196 & CDK7 & CDK7 & CMGC & CDK & CDK7 & 515462 \\
\hline 197 & CDK11 & CDC2L6 & CMGC & CDK & CDK8 & 511802 \\
\hline 198 & CDK8 & CDK8 & CMGC & CDK & CDK8 & 507149 \\
\hline 199 & CDK9 & CDK9 & CMGC & CDK & CDK9 & 520580 \\
\hline
\end{tabular}




\begin{tabular}{|c|c|c|c|c|c|c|}
\hline 200 & CHED & CDC2L5 & CMGC & CDK & CRK7 & 511147 \\
\hline 201 & CRK7 & CRKRS & CMGC & CDK & CRK7 & 511513 \\
\hline 202 & PITSLRE & CDC2L1 & CMGC & CDK & PITSLRE & 493708 \\
\hline 203 & PCTAIRE1 & PCTK1 & CMGC & CDK & TAIRE & 613810 \\
\hline 204 & PCTAIRE2 & PCTK2 & CMGC & CDK & TAIRE & 539655 \\
\hline 205 & PCTAIRE3 & PCTK3 & CMGC & CDK & TAIRE & 534048 \\
\hline 206 & PFTAIRE1 & CDK14 & CMGC & CDK & TAIRE & 784403 \\
\hline 207 & PFTAIRE2 & CDK15 & CMGC & CDK & TAIRE & 616118 \\
\hline 208 & CCRK & CCRK & CMGC & CDK & & 510920 \\
\hline 209 & CDKL1 & CDKL1 & CMGC & CDKL & & 523900 \\
\hline 210 & CDKL2 & CDKL2 & CMGC & CDKL & & 533114 \\
\hline 211 & CDKL3 & CDKL3 & CMGC & CDKL & & 541279 \\
\hline 212 & CDKL4 & CDKL4 & CMGC & CDKL & & 517478 \\
\hline 213 & CDKL5 & CDKL5 & CMGC & CDKL & & 538337 \\
\hline 214 & CLK1 & CLK1 & CMGC & CLK & & 613808 \\
\hline 215 & CLK2 & CLK2 & CMGC & CLK & & 512737 \\
\hline 216 & CLK3 & CLK3 & CMGC & CLK & & 505499 \\
\hline 217 & CLK4 & CLK4 & CMGC & CLK & & 529633 \\
\hline 218 & DYRK1A & DYRK1A & CMGC & DYRK & Dyrk1 & 532989 \\
\hline 219 & DYRK1B & DYRK1B & CMGC & DYRK & Dyrk1 & 507571 \\
\hline 220 & DYRK2 & DYRK2 & CMGC & DYRK & Dyrk2 & 514916 \\
\hline 221 & DYRK3 & DYRK3 & CMGC & DYRK & Dyrk2 & 505149 \\
\hline 222 & DYRK4 & LOC531276 & CMGC & DYRK & Dyrk2 & 531276 \\
\hline 223 & HIPK1 & HIPK1 & CMGC & DYRK & HIPK & 512233 \\
\hline 224 & HIPK2 & HIPK2 & CMGC & DYRK & HIPK & 510158 \\
\hline 225 & HIPK3 & HIPK3 & CMGC & DYRK & HIPK & 538866 \\
\hline 226 & HIPK4 & HIPK4 & CMGC & DYRK & HIPK & 520157 \\
\hline 227 & PRP4 & PRPF4B & CMGC & DYRK & PRP4 & 512236 \\
\hline 228 & GSK3A & GSK3A & CMGC & GSK & & 536561 \\
\hline 229 & GSK3B & GSK3B & CMGC & GSK & & 790875 \\
\hline 230 & Erk1 & MAPK3 & CMGC & MAPK & ERK & 531391 \\
\hline 231 & Erk2 & MAPK1 & CMGC & MAPK & ERK & 327672 \\
\hline 232 & Erk3 & MAPK6 & CMGC & MAPK & ERK & 538094 \\
\hline 233 & Erk4 & MAPK4 & CMGC & MAPK & ERK & 529183 \\
\hline 234 & Erk5 & MAPK7 & CMGC & MAPK & ERK & 537703 \\
\hline 235 & Erk7 & MAPK15 & CMGC & MAPK & Erk7 & 512125 \\
\hline 236 & JNK1 & MAPK8 & CMGC & MAPK & JNK & 539941 \\
\hline 237 & JNK2 & MAPK9 & CMGC & MAPK & JNK & 534125 \\
\hline 238 & JNK3 & MAPK10 & CMGC & MAPK & JNK & 537631 \\
\hline 239 & NLK & NLK & CMGC & MAPK & nmo & 507204 \\
\hline 240 & p38a & MAPK14 & CMGC & MAPK & p38 & 534492 \\
\hline 241 & p38b & MAPK11 & CMGC & MAPK & p38 & 618906 \\
\hline 242 & p38d & MAPK13 & CMGC & MAPK & p38 & 535327 \\
\hline 243 & p38g & MAPK12 & CMGC & MAPK & p38 & 512943 \\
\hline 244 & ICK & ICK & CMGC & RCK & & 506286 \\
\hline 245 & MAK & MAK & CMGC & RCK & & 536048 \\
\hline 246 & MOK & RAGE & CMGC & RCK & & 616277 \\
\hline 247 & MSSK1 & SRPK3 & CMGC & SRPK & & 508325 \\
\hline 248 & SRPK1 & SRPK1 & CMGC & SRPK & & 512631 \\
\hline 249 & SRPK2 & SRPK2 & CMGC & SRPK & & 505945 \\
\hline
\end{tabular}




\begin{tabular}{|c|c|c|c|c|c|c|}
\hline 250 & AurA & AURKA & Other & AUR & & 504437 \\
\hline 251 & AurB & AURKB & Other & AUR & & 360192 \\
\hline 252 & AurC & AURKC & Other & AUR & & 618599 \\
\hline 253 & AurL & & Other & AUR & & \\
\hline 254 & BUB1 & BUB1 & Other & BUB & & 514777 \\
\hline 255 & BUBR1 & BUB1B & Other & BUB & & 537027 \\
\hline 256 & PRPK & TP53RK & Other & Bud32 & & 619100 \\
\hline 257 & CaMKK1 & CAMKK1 & Other & CAMKK & Meta & 510260 \\
\hline 258 & CaMKK2 & CAMKK2 & Other & CAMKK & Meta & 509084 \\
\hline 259 & CDC7 & CDC7 & Other & CDC7 & & 516094 \\
\hline 260 & CK2a1 & CSNK2A1 & Other & CK2 & & 282419 \\
\hline 261 & CK2a2 & CSNK2A2 & Other & CK2 & & 282420 \\
\hline 262 & Haspin & GSG2 & Other & Haspin & & 767819 \\
\hline 263 & IKKa & CHUK & Other & IKK & & 281073 \\
\hline 264 & $\mathrm{IKKb}$ & IKBKB & Other & IKK & & 281854 \\
\hline 265 & IKKe & IKBKE & Other & IKK & & 533216 \\
\hline 266 & TBK1 & TBK1 & Other & IKK & & 533141 \\
\hline 267 & IRE1 & IRE1 & Other & IRE & & \\
\hline 268 & IRE2 & IRE2 & Other & IRE & & \\
\hline 269 & MOS & MOS & Other & MOS & & 444869 \\
\hline 270 & AAK1 & AAK1 & Other & NAK & & 532546 \\
\hline 271 & BIKE & BIKE & Other & NAK & & 505766 \\
\hline 272 & GAK & GAK & Other & NAK & & 511296 \\
\hline 273 & MPSK1 & STK16 & Other & NAK & & 521237 \\
\hline 274 & NEK1 & & Other & NEK & & \\
\hline 275 & NEK10 & NEK10 & Other & NEK & & 522335 \\
\hline 276 & NEK11 & NEK11 & Other & NEK & & 614924 \\
\hline 277 & NEK2 & NEK2 & Other & NEK & & 523998 \\
\hline 278 & NEK3 & NEK3 & Other & NEK & & 505876 \\
\hline 279 & NEK4 & NEK4 & Other & NEK & & 511455 \\
\hline 280 & NEK5 & NEK5 & Other & NEK & & 100138147 \\
\hline 281 & NEK6 & NEK6 & Other & NEK & & 515816 \\
\hline 282 & NEK7 & NEK7 & Other & NEK & & 781355 \\
\hline 283 & NEK8 & NEK8 & Other & NEK & & 532332 \\
\hline 284 & NEK9 & NEK9 & Other & NEK & & 534652 \\
\hline 285 & SBK & LOC614815 & Other & NKF1 & & 614815 \\
\hline 286 & SgK069 & SBK2 & Other & NKF1 & & 615123 \\
\hline 287 & SgK110 & LOC789661 & Other & NKF1 & & 789661 \\
\hline 288 & PINK1 & PINK1 & Other & NKF2 & & 510683 \\
\hline 289 & SgK223 & SgK223 & Other & NKF3 & & \\
\hline 290 & SgK269 & SgK269 & Other & NKF3 & & \\
\hline 291 & CLIK1 & STK35 & Other & NKF4 & & 540979 \\
\hline 292 & CLIK1L & PDIK1L & Other & NKF4 & & 510253 \\
\hline 293 & SgK424 & LOC785881 & Other & NKF5 & & 785881 \\
\hline 294 & TEX14 & TEX14 & Other & NKF5 & & 522810 \\
\hline 295 & NRBP1 & NRBP1 & Other & NRBP & & 532919 \\
\hline 296 & NRBP2 & NRBP2 & Other & NRBP & & 504664 \\
\hline 297 & KIS & UHMK1 & Other & Other-Unique & & 538770 \\
\hline 298 & RNAseL & RNASEL & Other & Other-Unique & & 100048947 \\
\hline 299 & SgK071 & LOC614899 & Other & Other-Unique & & 614899 \\
\hline
\end{tabular}




\begin{tabular}{|c|c|c|c|c|c|c|}
\hline 300 & SgK196 & MGC151666 & Other & Other-Unique & & 514490 \\
\hline 301 & SgK396 & STK31 & Other & Other-Unique & & 781749 \\
\hline 302 & SgK493 & PKDCC & Other & Other-Unique & & 539467 \\
\hline 303 & SgK496 & RIPK5 & Other & Other-Unique & & 534684 \\
\hline 304 & GCN2 & GCN2 & Other & PEK & GCN2 & \\
\hline 305 & PEK & EIF2AK3 & Other & PEK & PEK & 535820 \\
\hline 306 & $\mathrm{HRI}$ & EIF2AK1 & Other & PEK & & 507689 \\
\hline 307 & PKR & EIF2AK2 & Other & PEK & & 347700 \\
\hline 308 & PLK1 & PLK1 & Other & PLK & & 538238 \\
\hline 309 & PLK2 & PLK2 & Other & PLK & & 539449 \\
\hline 310 & PLK3 & PLK3 & Other & PLK & & 504282 \\
\hline 311 & PLK4 & PLK4 & Other & PLK & & 514405 \\
\hline 312 & PLK5 & LOC511891 & Other & PLK & & 511891 \\
\hline 313 & SCYL1 & SCYL1 & Other & SCY1 & & 515247 \\
\hline 314 & SCYL2 & SCYL2 & Other & SCY1 & & 782059 \\
\hline 315 & SCYL3 & SCYL3 & Other & SCY1 & & 506600 \\
\hline 316 & Slob & PXK & Other & Slob & & 614093 \\
\hline 317 & TBCK & TBCK & Other & TBCK & & 528650 \\
\hline 318 & TLK1 & TLK1 & Other & TLK & & 537517 \\
\hline 319 & TLK2 & TLK2 & Other & TLK & & 534705 \\
\hline 320 & PBK & PBK & Other & TOPK & & 534781 \\
\hline 321 & TTK & TTK & Other & TTK & & 524925 \\
\hline 322 & Fused & STK36 & Other & ULK & & 508408 \\
\hline 323 & ULK1 & ULK1 & Other & ULK & & 526310 \\
\hline 324 & ULK2 & ULK2 & Other & ULK & & 618601 \\
\hline 325 & ULK3 & ULK3 & Other & ULK & & 506063 \\
\hline 326 & ULK4 & ULK4 & Other & ULK & & 531267 \\
\hline 327 & PIK3R4 & PIK3R4 & Other & VPS15 & & 507414 \\
\hline 328 & MYT1 & PKMYT1 & Other & WEE & & 522613 \\
\hline 329 & Wee1 & WEE1 & Other & WEE & & 540322 \\
\hline 330 & Wee1B & WEE2 & Other & WEE & & 520049 \\
\hline 331 & Wnk1 & WNK1 & Other & Wnk & & 506433 \\
\hline 332 & Wnk2 & WNK2 & Other & Wnk & & 506520 \\
\hline 333 & Wnk3 & WNK3 & Other & Wnk & & 533409 \\
\hline 334 & Wnk4 & WNK4 & Other & Wnk & & \\
\hline 335 & $\mathrm{ANPa}$ & NPR1 & RGC & RGC & & 533048 \\
\hline 336 & $\mathrm{ANPb}$ & NPR2 & RGC & RGC & & 281357 \\
\hline 337 & CYGD & GUCY2D & RGC & RGC & & 282245 \\
\hline 338 & CYGF & GUCY2F & RGC & RGC & & 282246 \\
\hline 339 & HSER & GUCY2C & RGC & RGC & & 282244 \\
\hline 340 & MAP3K1 & MAP3K1 & STE & STE11 & & 523962 \\
\hline 341 & MAP3K2 & MAP3K2 & STE & STE11 & & 504592 \\
\hline 342 & MAP3К3 & MAP3К3 & STE & STE11 & & 508943 \\
\hline 343 & MAP3K4 & MAP3K4 & STE & STE11 & & 511779 \\
\hline 344 & MAP3K5 & MAP3K5 & STE & STE11 & & 537380 \\
\hline 345 & MAP3K6 & MAP3K6 & STE & STE11 & & 614598 \\
\hline 346 & MAP3K7 & MAP3K15 & STE & STE11 & & 529335 \\
\hline 347 & MAP3К8 & YSK4 & STE & STE11 & & 538897 \\
\hline 348 & OSR1 & OSR1 & STE & STE20 & FRAY & \\
\hline 349 & STLK3 & STK39 & STE & STE20 & FRAY & 522103 \\
\hline
\end{tabular}




\begin{tabular}{|c|c|c|c|c|c|c|}
\hline 350 & GCK & MAP4K2 & STE & STE20 & KHS & 520058 \\
\hline 351 & HPK1 & MAP4K1 & STE & STE20 & KHS & 522002 \\
\hline 352 & KHS1 & MAP4K5 & STE & STE20 & KHS & 781335 \\
\hline 353 & KHS2 & MAP4K3 & STE & STE20 & KHS & 507624 \\
\hline 354 & ZC1 & ZC1 & STE & STE20 & MSN & \\
\hline 355 & $\mathrm{ZC2}$ & TNIK & STE & STE20 & $\mathrm{MSN}$ & 539627 \\
\hline 356 & ZC3 & MINK1 & STE & STE20 & MSN & 519558 \\
\hline 357 & ZC4 & NRK & STE & STE20 & MSN & 783774 \\
\hline 358 & MST1 & STK4 & STE & STE20 & MST & 514886 \\
\hline 359 & MST2 & STK3 & STE & STE20 & MST & 533815 \\
\hline 360 & MYO3A & MYO3A & STE & STE20 & NinaC & 535824 \\
\hline 361 & MYO3B & MYO3B & STE & STE20 & NinaC & 536123 \\
\hline 362 & PAK1 & PAK1 & STE & STE20 & PAKA & 533729 \\
\hline 363 & PAK2 & LOC513673 & STE & STE20 & PAKA & 513673 \\
\hline 364 & PAK3 & PAK3 & STE & STE20 & PAKA & 534526 \\
\hline 365 & PAK4 & PAK4 & STE & STE20 & PAKB & 539742 \\
\hline 366 & PAK5 & PAK7 & STE & STE20 & PAKB & 513432 \\
\hline 367 & PAK6 & PAK6 & STE & STE20 & PAKB & 539224 \\
\hline 368 & LOK & STK10 & STE & STE20 & SLK & 526376 \\
\hline 369 & SLK & SLK & STE & STE20 & SLK & 535342 \\
\hline 370 & STLK5 & LYK5 & STE & STE20 & STLK & 515024 \\
\hline 371 & STLK6 & STRADB & STE & STE20 & STLK & 506029 \\
\hline 372 & TAO1 & TAOK1 & STE & STE20 & TAO & 536532 \\
\hline 373 & TAO2 & TAOK2 & STE & STE20 & TAO & 533897 \\
\hline 374 & TAO3 & TAOK3 & STE & STE20 & TAO & 534620 \\
\hline 375 & MST3 & STK24-LIKE & STE & STE20 & YSK & 532455 \\
\hline 376 & MST4 & MST4 & STE & STE20 & YSK & 539445 \\
\hline 377 & YSK1 & STK25 & STE & STE20 & YSK & 373543 \\
\hline 378 & MAP2K1 & MAP2K1 & STE & STE7 & & 533199 \\
\hline 379 & MAP2K2 & MAP2K2 & STE & STE7 & & 510434 \\
\hline 380 & MAP2K3 & MAP2K3 & STE & STE7 & & 516039 \\
\hline 381 & MAP2K4 & MAP2K4 & STE & STE7 & & 526469 \\
\hline 382 & MAP2K5 & MAP2K5 & STE & STE7 & & 100295732 \\
\hline 383 & MAP2K6 & MAP2K6 & STE & STE7 & & 286883 \\
\hline 384 & MAP2K7 & MAP2K7 & STE & STE7 & & 787278 \\
\hline 385 & COT & MAP3K8 & STE & STE-Unique & & 535622 \\
\hline 386 & NIK & MAP3K14 & STE & STE-Unique & & 508367 \\
\hline 387 & $A B L$ & ABL1 & TK & Abl & & 540876 \\
\hline 388 & ARG & $A B L 2$ & TK & Abl & & 511845 \\
\hline 389 & ACK & ACK1 & TK & Ack & & 280710 \\
\hline 390 & TNK1 & TNK1 & TK & Ack & & 510356 \\
\hline 391 & ALK & ALK & TK & Alk & & 536642 \\
\hline 392 & AXL & AXL & TK & $A x \mid$ & & 516598 \\
\hline 393 & MER & MERTK & TK & $A x I$ & & 504429 \\
\hline 394 & TYRO3 & TYRO3 & TK & $A x I$ & & 788224 \\
\hline 395 & CCK4 & PTK7 & TK & CCK4 & & 514819 \\
\hline 396 & CSK & CSK & TK & Csk & & 509246 \\
\hline 397 & CTK & MATK & TK & Csk & & 504668 \\
\hline 398 & DDR1 & DDR1 & TK & DDR & & 534092 \\
\hline 399 & DDR2 & DDR2 & TK & DDR & & 533523 \\
\hline
\end{tabular}




\begin{tabular}{|c|c|c|c|c|c|}
\hline 400 & EGFR & EGFR & TK & EGFR & 407217 \\
\hline 401 & ErbB2 & ERBB2 & TK & EGFR & \\
\hline 402 & ErbB3 & ERBB3 & TK & EGFR & 785655 \\
\hline 403 & ErbB4 & ERBB4 & TK & EGFR & 522419 \\
\hline 404 & EphA1 & EPHA1 & TK & Eph & 525946 \\
\hline 405 & EphA10 & EPHA10 & TK & Eph & 540439 \\
\hline 406 & EphA2 & EPHA2 & TK & Eph & 512798 \\
\hline 407 & EphA3 & EPHA3 & TK & Eph & 537951 \\
\hline 408 & EphA4 & EPHA4 & TK & Eph & 520192 \\
\hline 409 & EphA5 & EPHA5 & TK & Eph & 538224 \\
\hline 410 & EphA6 & EPHA6 & TK & Eph & 616165 \\
\hline 411 & EphA7 & EPHA7 & TK & Eph & 538797 \\
\hline 412 & EphA8 & EPHA8 & TK & Eph & 517368 \\
\hline 413 & EphB1 & EPHB1 & TK & Eph & 534731 \\
\hline 414 & EphB1L & LOC618263 & TK & Eph & 618263 \\
\hline 415 & EphB2 & EPHB2 & TK & Eph & 535137 \\
\hline 416 & EphB3 & EPHB3 & TK & Eph & 540907 \\
\hline 417 & EphB4 & EPHB4 & TK & Eph & 515756 \\
\hline 418 & EphB6 & EPHB6 & TK & Eph & 529800 \\
\hline 419 & FAK & PTK2 & TK & Fak & 506270 \\
\hline 420 & PYK2 & PTK2B & TK & Fak & 541008 \\
\hline 421 & FER & FER & TK & Fer & 536759 \\
\hline 422 & FERL & & TK & Fer & \\
\hline 423 & FES & FES & TK & Fer & 507304 \\
\hline 424 & FGFR1 & FGFR1 & TK & FGFR & 281768 \\
\hline 425 & FGFR2 & FGFR2 & TK & FGFR & 404193 \\
\hline 426 & FGFR3 & FGFR3 & TK & FGFR & 281769 \\
\hline 427 & FGFR4 & FGFR4 & TK & FGFR & 317696 \\
\hline 428 & IGF1R & IGF1R & TK & InsR & 281848 \\
\hline 429 & INSR & INSR & TK & InsR & 408017 \\
\hline 430 & IRR & INSRR & TK & InsR & 787068 \\
\hline 431 & JAK1 & JAK1 & TK & JakA & 537201 \\
\hline 432 & JAK2 & JAK2 & TK & JakA & 525246 \\
\hline 433 & JAK3 & JAK3 & TK & JakA & 538276 \\
\hline 434 & TYK2 & TYK2 & TK & JakA & 512484 \\
\hline 435 & LMR1 & AATK & TK & Lmr & 511515 \\
\hline 436 & LMR2 & LMTK2 & TK & $\mathrm{Lmr}$ & 512290 \\
\hline 437 & LMR3 & LMR3 & TK & Lmr & \\
\hline 438 & MET & MET & TK & Met & 280855 \\
\hline 439 & RON & MST1R & TK & Met & 525504 \\
\hline 440 & MUSK & MUSK & TK & Musk & 540052 \\
\hline 441 & FLT3 & FLT3 & TK & PDGFR & 512700 \\
\hline 442 & FMS & FMS & TK & PDGFR & \\
\hline 443 & KIT & KIT & TK & PDGFR & 280832 \\
\hline 444 & PDGFRa & PDGFRA & TK & PDGFR & 282301 \\
\hline 445 & PDGFRb & PDGFRB & TK & PDGFR & 527165 \\
\hline 446 & RET & RET & TK & Ret & 515924 \\
\hline 447 & ROR1 & ROR1 & TK & Ror & 783965 \\
\hline 448 & ROR2 & ROR2 & TK & Ror & 785924 \\
\hline 449 & RYK & RYK & TK & Ryk & 781353 \\
\hline
\end{tabular}




\begin{tabular}{|c|c|c|c|c|c|c|}
\hline 450 & RYKL & & TK & Ryk & & \\
\hline 451 & BLK & BLK & TK & Src & & 532587 \\
\hline 452 & BRK & PTK6 & TK & Src & & 529814 \\
\hline 453 & FGR & FGR & TK & Src & & 516133 \\
\hline 454 & FRK & FRK & TK & Src & & 509227 \\
\hline 455 & FYN & FYN & TK & Src & & 527263 \\
\hline 456 & $\mathrm{HCK}$ & $\mathrm{HCK}$ & TK & Src & & 280814 \\
\hline 457 & LCK & LCK & TK & Src & & 508890 \\
\hline 458 & LYN & LYN & TK & Src & & 534996 \\
\hline 459 & SRC & SRC & TK & Src & & 535742 \\
\hline 460 & SRM & SRMS & TK & Src & & 521816 \\
\hline 461 & YES & YES1 & TK & Src & & 507632 \\
\hline 462 & SYK & SYK & TK & Syk & & 515515 \\
\hline 463 & ZAP70 & ZAP70 & TK & Syk & & 504509 \\
\hline 464 & BMX & $\mathrm{BMX}$ & TK & Tec & & 531514 \\
\hline 465 & BTK & BTK & TK & Tec & & 533459 \\
\hline 466 & ITK & ITK & TK & Tec & & 522311 \\
\hline 467 & TEC & TEC & TK & Tec & & 504733 \\
\hline 468 & TXK & TXK & TK & Tec & & 504782 \\
\hline 469 & TIE1 & TIE1 & TK & Tie & & 280941 \\
\hline 470 & TIE2 & TEK & TK & Tie & & 280939 \\
\hline 471 & SuRTK106 & STYK1 & TK & TK-Unique & & 513920 \\
\hline 472 & TRKA & NTRK1 & TK & Trk & & 353111 \\
\hline 473 & TRKB & TRKB & TK & Trk & & \\
\hline 474 & TRKC & NTRK3 & TK & Trk & & 539126 \\
\hline 475 & FLT1 & FLT1 & TK & VEGFR & & 503620 \\
\hline 476 & FLT4 & FLT4 & TK & VEGFR & & 338031 \\
\hline 477 & KDR & KDR & TK & VEGFR & & 407170 \\
\hline 478 & IRAK1 & IRAK1 & TKL & IRAK & & 533953 \\
\hline 479 & IRAK2 & IRAK2 & TKL & IRAK & & 515034 \\
\hline 480 & IRAK3 & IRAK3 & TKL & IRAK & & 510342 \\
\hline 481 & IRAK4 & IRAK4 & TKL & IRAK & & 533692 \\
\hline 482 & LIMK1 & LIMK1 & TKL & LISK & LIMK & 535225 \\
\hline 483 & LIMK2 & LIMK2 & TKL & LISK & LIMK & 513539 \\
\hline 484 & TESK1 & TESK1 & TKL & LISK & TESK & 527643 \\
\hline 485 & TESK2 & TESK2 & TKL & LISK & TESK & 539979 \\
\hline 486 & LRRK1 & LRRK1 & TKL & LRRK & & 513994 \\
\hline 487 & LRRK2 & LRRK2 & TKL & LRRK & & 535652 \\
\hline 488 & $\mathrm{HH} 498$ & TNNI3K & TKL & MLK & $\mathrm{HH} 498$ & 535940 \\
\hline 489 & DLK & DLK & TKL & MLK & LZK & \\
\hline 490 & LZK & MAP3K13 & TKL & MLK & LZK & 505369 \\
\hline 491 & MLK1 & LOC538340 & TKL & MLK & MLK & 538340 \\
\hline 492 & MLK2 & MAP3K10 & TKL & MLK & MLK & 781037 \\
\hline 493 & MLK3 & MAP3K11 & TKL & MLK & MLK & 514210 \\
\hline 494 & MLK4 & LOC616014 & TKL & MLK & MLK & 616014 \\
\hline 495 & ZAK & ZAK & TKL & MLK & MLK & 519251 \\
\hline 496 & TAK1 & MAP3K7 & TKL & MLK & TAK1 & 529146 \\
\hline 497 & ARAF & ARAF & TKL & RAF & & 540421 \\
\hline 498 & BRAF & BRAF & TKL & RAF & & 536051 \\
\hline 499 & KSR1 & KSR1 & TKL & RAF & & 100138565 \\
\hline
\end{tabular}




\begin{tabular}{|c|c|c|c|c|c|c|}
\hline 500 & KSR2 & KSR2 & TKL & RAF & & 617325 \\
\hline 501 & RAF1 & RAF1 & TKL & RAF & & 521196 \\
\hline 502 & ANKRD3 & RIPK4 & TKL & RIPK & & 508668 \\
\hline 503 & RIPK1 & RIPK1 & TKL & RIPK & & 504727 \\
\hline 504 & RIPK2 & RIPK2 & TKL & RIPK & & 534407 \\
\hline 505 & RIPK3 & RIPK3 & TKL & RIPK & & 507427 \\
\hline 506 & SgK288 & SgK288 & TKL & RIPK & & \\
\hline 507 & ALK1 & ACVRL1 & TKL & STKR & Type1 & 534536 \\
\hline 508 & ALK2 & ACVR1 & TKL & STKR & Type1 & 338068 \\
\hline 509 & ALK4 & ACVR1B & TKL & STKR & Type1 & 539315 \\
\hline 510 & ALK7 & ACVR1C & TKL & STKR & Type1 & 536380 \\
\hline 511 & BMPR1A & BMPR1A & TKL & STKR & Type1 & 404101 \\
\hline 512 & BMPR1B & BMPR1B & TKL & STKR & Type1 & 407128 \\
\hline 513 & TGFbR1 & TGFBR1 & TKL & STKR & Type1 & 282382 \\
\hline 514 & ACTR2 & ACVR2A & TKL & STKR & Type2 & 281598 \\
\hline 515 & ACTR2B & ACVR2B & TKL & STKR & Type2 & 282131 \\
\hline 516 & BMPR2 & BMPR2 & TKL & STKR & Type2 & 407127 \\
\hline 517 & MISR2 & & TKL & STKR & Type2 & \\
\hline 518 & TGFbR2 & TGFBR2 & TKL & STKR & Type2 & 535376 \\
\hline 519 & ILK & ILK & TKL & TKL-Unique & ILK & 540207 \\
\hline 520 & MLKL & MLKL & TKL & TKL-Unique & & 790225 \\
\hline 521 & Par-1L & & TKL & TKL-Unique & & \\
\hline
\end{tabular}




\begin{tabular}{|c|c|c|}
\hline Protein Acc & RNA acc & Synonyms \\
\hline NP_776411.1 & NM_173986.2 & - \\
\hline XP_870006.1 & XM_864913.2 & - \\
\hline NP_001178238.1 & NM_001191309.1 & \\
\hline NP_001095972.1 & NM_001102502.1 & MGC155045 \\
\hline XP_618596.3 & XM_618596.3 & - \\
\hline XP_001253770.1 & XM_001253769.1 & - \\
\hline NP_776877.1 & NM_174452.2 & - \\
\hline NP_777135.1 & NM_174710.2 & GRK2 \\
\hline NP_776925.1 & NM_174500.2 & - \\
\hline XP_590012.3 & XM_590012.3 & - \\
\hline NP_776756.1 & NM_174331.2 & GPRK5 \\
\hline NP_776757.1 & NM_174332.3 & GPRK7 \\
\hline NP_776598.1 & NM_174173.2 & RHOK \\
\hline XP_002704808.1 & XM_002704762.1 & \\
\hline XP_613922.3 & XM_613922.3 & - \\
\hline NP_001107237.1 & NM_001113765.1 & - \\
\hline XP_616050.3 & XM_616050.3 & - \\
\hline XP_584953.3 & XM_584953.3 & - \\
\hline NP_001075071.1 & NM_001081602.1 & MGC155151 \\
\hline NP_001094562.1 & NM_001101092.1 & - \\
\hline NP_777009.1 & NM_174584.2 & - \\
\hline NP_777010.1 & NM_174585.2 & - \\
\hline XP_582115.3 & XM_582115.3 & - \\
\hline XP_001788715.1 & XM_001788663.2 & \\
\hline XP_606901.3 & XM_606901.3 & - \\
\hline NP_001071301.1 & NM_001077833.1 & - \\
\hline NP_776860.1 & NM_174435.1 & PKRCA \\
\hline NP_777012.1 & NM_174587.1 & - \\
\hline XP_602125.3 & XM_602125.3 & - \\
\hline NP_001071323.1 & NM_001077855.1 & - \\
\hline NP_001104590.1 & NM_001111120.1 & - \\
\hline NP_001070331.1 & NM_001076863.1 & - \\
\hline XP_582266.3 & XM_582266.3 & - \\
\hline NP_776861.1 & NM_174436.2 & CGKI|cGKI-beta \\
\hline XP_612707.3 & XM_612707.3 & - \\
\hline NP_001073715.1 & NM_001080246.1 & MGC142408 \\
\hline XP_883499.2 & XM_878406.2 & - \\
\hline XP_581630.2 & XM_581630.3 & - \\
\hline XP_580522.3 & XM_580522.3 & MSK1 \\
\hline NP_001178329.1 & NM_001191400.1 & \\
\hline NP_991385.1 & NM_205816.1 & - \\
\hline XP_582478.3 & XM_582478.3 & - \\
\hline
\end{tabular}




\begin{tabular}{|c|c|c|}
\hline XP_596135.3 & XM_596135.3 & - \\
\hline NP_001092407.1 & NM_001098937.1 & MGC160087 \\
\hline NP_001077191.1 & NM_001083722.1 & MGC139400 \\
\hline XP_604590.3 & XM_604590.3 & - \\
\hline XP_870724.1 & XM_865631.2 & - \\
\hline NP_001032549.1 & NM_001037472.1 & MGC128730 \\
\hline XP_606198.2 & XM_606198.3 & - \\
\hline NP_001095503.1 & NM_001102033.1 & MGC166421 \\
\hline NP_001069228.1 & NM_001075760.1 & MGC139463 \\
\hline XP_870723.1 & XM_865630.2 & - \\
\hline NP_001098517.1 & NM_001105047.1 & MGC148827 \\
\hline XP_607574.3 & XM_607574.3 & - \\
\hline XP_602539.3 & XM_602539.3 & - \\
\hline NP_001020491.1 & NM_001025320.1 & MGC137976 \\
\hline NP_001098794.1 & NM_001105324.1 & PTK9L|MGC148415 \\
\hline NP_001039884.1 & NM_001046419.1 & MGC137459 \\
\hline XP_001790539.1 & XM_001790487.1 & \\
\hline NP_001070363.1 & NM_001076895.1 & MGC142877 \\
\hline NP_001094580.1 & NM_001101110.1 & \\
\hline XP_586554.2 & XM_586554.3 & \\
\hline XP_870587.2 & XM_865494.3 & \\
\hline XP_605991.3 & XM_605991.3 & \\
\hline XP_599997.2 & XM_599997.3 & - \\
\hline XP_587341.3 & XM_587341.4 & \\
\hline XP_602698.4 & XM_602698.4 & \\
\hline XP_001256514.1 & XM_001256513.1 & \\
\hline NP_001039331.1 & NM_001045866.1 & \\
\hline NP_001070341.1 & NM_001076873.1 & MGC142472 \\
\hline XP_002704782.1 & XM_002704736.1 & \\
\hline NP_001069320.1 & NM_001075852.1 & MGC151933 \\
\hline NP_001030249.2 & NM_001035077.2 & \\
\hline NP_001091466.1 & NM_001097997.1 & \\
\hline NP_001014955.1 & NM_001014955.1 & PTK9 \\
\hline NP_001039371.1 & NM_001045906.1 & MGC127910 \\
\hline XP_607085.4 & XM_607085.4 & \\
\hline NP_001069321.1 & NM_001075853.1 & \\
\hline NP_001095404.1 & NM_001101934.1 & MGC166016 \\
\hline NP_001095353.1 & NM_001101883.1 & MGC166250 \\
\hline XP_605200.3 & XM_605200.4 & \\
\hline XP_581054.3 & XM_581054.4 & \\
\hline XP_879315.2 & XM_874222.3 & \\
\hline XP_002694089.1 & XM_002694043.1 & \\
\hline XP_603490.3 & XM_603490.4 & \\
\hline XP_583735.3 & XM_583735.3 & \\
\hline XP_594442.2 & XM_594442.3 & - \\
\hline NP_001178220.1 & NM_001191291.1 & \\
\hline NP_001069304.1 & NM_001075836.1 & MGC138071 \\
\hline XP_580963.3 & XM_580963.3 & \\
\hline XP_001250500.1 & XM_001250499.2 & \\
\hline
\end{tabular}




\begin{tabular}{|c|c|c|}
\hline XP_617403.3 & XM_617403.4 & \\
\hline NP_001070336.1 & NM_001076868.1 & MGC142688 \\
\hline NP_001069787.1 & NM_001076319.1 & MGC142416 \\
\hline XP_002692107.1 & XM_002692061.1 & - \\
\hline NP_001070304.1 & NM_001076836.1 & MGC142748 \\
\hline NP_001069406.1 & NM_001075938.1 & MGC139375|MGC155201 \\
\hline NP_001030434.1 & NM_001035357.1 & MGC128545 \\
\hline NP_001039798.1 & NM_001046333.1 & MGC128576 \\
\hline NP_001091033.1 & NM_001097564.1 & - \\
\hline XP_002691060.1 & XM_002691014.1 & - \\
\hline NP_001103272.1 & NM_001109802.1 & MGC165837 \\
\hline XP_583885.3 & XM_583885.3 & - \\
\hline XP_618200.3 & XM_618200.3 & - \\
\hline XP_615982.3 & XM_615982.3 & - \\
\hline NP_001091492.1 & NM_001098023.1 & - \\
\hline$\overline{X P} 617816.3$ & XM_617816.3 & - \\
\hline XP_593229.3 & XM_593229.3 & - \\
\hline XP_588081.3 & XM_588081.3 & - \\
\hline NP_001095652.1 & NM_001102182.1 & MGC140219 \\
\hline XP_614792.3 & XM_614792.3 & - \\
\hline NP_001030436.1 & NM_001035359.1 & - \\
\hline XP_875908.2 & XM_870815.2 & - \\
\hline NP_001104730.1 & NM_001111260.1 & - \\
\hline XP_608675.2 & XM_608675.3 & - \\
\hline XP_001789835.1 & XM_001789783.1 & \\
\hline XP_001256004.1 & XM_001256003.1 & - \\
\hline XP_589604.3 & XM_589604.3 & - \\
\hline XP_588111.3 & XM_588111.3 & - \\
\hline XP_582999.3 & XM_582999.3 & - \\
\hline XP_002702780.1 & XM_002702734.1 & \\
\hline NP_001069195.1 & NM_001075727.1 & MGC139116 \\
\hline NP_001069376.1 & NM_001075908.1 & MGC139553 \\
\hline NP_001069344.1 & NM_001075876.1 & MGC142730 \\
\hline NP_001095951.1 & NM_001102481.1 & MGC139144 \\
\hline XP_613544.3 & XM_613544.3 & - \\
\hline XP_002690922.1 & XM_002690876.1 & - \\
\hline NP_001094594.1 & NM_001101124.1 & - \\
\hline NP_001076891.1 & NM_001083422.1 & MGC140096 \\
\hline NP_001033169.1 & NM_001038080.1 & MGC134048 \\
\hline XP_616231.3 & XM_616231.3 & - \\
\hline XP_583775.3 & XM_583775.3 & - \\
\hline XP_001255255.1 & XM_001255254.1 & - \\
\hline NP_001029951.1 & NM_001034779.1 & MGC127030 \\
\hline XP_615733.3 & XM_615733.3 & - \\
\hline NP_001030435.1 & NM_001035358.1 & MGC133983 \\
\hline XP_580870.3 & XM_580870.3 & - \\
\hline XP_604749.3 & XM_604749.3 & - \\
\hline
\end{tabular}




\begin{tabular}{|c|c|c|}
\hline XP_596207.3 & XM_596207.3 & - \\
\hline NP_001077188.1 & NM_001083719.1 & MGC155182 \\
\hline NP_788809.1 & NM_176636.2 & - \\
\hline XP_002703630.1 & XM_002703584.1 & \\
\hline NP_001039951.1 & NM_001046486.1 & MGC137450 \\
\hline NP_001039593.1 & NM_001046128.1 & MGC129049 \\
\hline NP_776569.1 & NM_174144.2 & - \\
\hline XP_585196.3 & XM_585196.3 & - \\
\hline XP_002688056.1 & XM_002688010.1 & \\
\hline XP_612625.3 & XM_612625.3 & - \\
\hline XP_001251452.1 & XM_001251451.1 & - \\
\hline XP_580387.3 & XM_580387.3 & - \\
\hline NP_001068881.1 & NM_001075413.1 & - \\
\hline NP_001029703.1 & NM_001034531.1 & - \\
\hline NP_001094575.1 & NM_001101105.1 & - \\
\hline NP_847887.2 & NM_178317.3 & TRB-2|TRB2 \\
\hline NP_001069571.1 & NM_001076103.1 & MGC142660 \\
\hline XP_585000.3 & XM_585000.3 & - \\
\hline XP_873585.2 & XM_868492.2 & - \\
\hline NP_001178293.1 & NM_001191364.1 & \\
\hline NP_001095682.1 & NM_001102212.1 & MGC154976 \\
\hline XP_588888.3 & XM_588888.3 & - \\
\hline NP_001077179.1 & NM_001083710.1 & TSSK2 \\
\hline XP_581847.2 & XM_581847.3 & - \\
\hline XP_870097.1 & XM_865004.2 & - \\
\hline XP_601315.3 & XM_601315.3 & - \\
\hline NP_777136.1 & NM_174711.2 & MGC133860 \\
\hline NP_001091628.1 & NM_001098159.1 & \\
\hline NP_001095550.1 & NM_001102080.1 & MGC166392 \\
\hline NP_001071577.1 & NM_001078109.1 & MGC143296 \\
\hline NP_001033235.1 & NM_001038146.1 & MGC134312 \\
\hline NP_001094526.1 & NM_001101056.1 & - \\
\hline XP_615092.2 & XM_615092.3 & - \\
\hline NP_001033313.1 & NM_001038224.1 & MGC128186 \\
\hline NP_001069319.1 & NM_001075851.1 & - \\
\hline NP_001039716.1 & NM_001046251.1 & MGC133825 \\
\hline NP_776441.1 & NM_174016.2 & MGC134454 \\
\hline NP_001014934.1 & NM_001014934.1 & - \\
\hline NP_001092648.1 & NM_001099178.1 & MGC160000 \\
\hline NP_001033666.1 & NM_001038577.2 & MGC134436 \\
\hline NP_001032683.1 & NM_001037594.1 & MGC133903 \\
\hline XP_589151.3 & XM_589151.3 & - \\
\hline NP_776442.1 & NM_174017.2 & MGC140720 \\
\hline NP_001069183.1 & NM_001075715.1 & MGC140417 \\
\hline XP_589209.3 & XM_589209.3 & - \\
\hline XP_583707.3 & XM_583707.3 & - \\
\hline NP_001014935.2 & NM_001014935.2 & MGC139578 \\
\hline
\end{tabular}




\begin{tabular}{|c|c|c|}
\hline XP_001251167.1 & XM_001251166.1 & - \\
\hline XP_588861.2 & XM_588861.3 & - \\
\hline NP_001007812.2 & NM_001007811.2 & \\
\hline NP_001094696.1 & NM_001101226.1 & - \\
\hline XP_588580.3 & XM_588580.3 & - \\
\hline NP_001076942.1 & NM_001083473.1 & MGC155091 \\
\hline XP_001789714.2 & XM_001789662.2 & PFTK1 \\
\hline XP_873177.3 & XM_868084.3 & PFTK2 \\
\hline NP_001092403.1 & NM_001098933.1 & MGC154961|MGC166397 \\
\hline NP_001094586.1 & NM_001101116.1 & - \\
\hline NP_001096744.1 & NM_001103274.1 & MGC142906 \\
\hline NP_001179783.1 & NM_001192854.1 & - \\
\hline NP_001033218.1 & NM_001038129.1 & MGC133738 \\
\hline XP_002700425.1 & XM_002700379.1 & \\
\hline NP_001095741.1 & NM_001102271.1 & MGC148790 \\
\hline XP_874270.2 & XM_869177.2 & - \\
\hline NP_001029420.1 & NM_001034248.1 & MGC128892 \\
\hline XP_870005.1 & XM_864912.2 & - \\
\hline XP_612233.2 & XM_612233.3 & - \\
\hline NP_001074984.1 & NM_001081515.1 & DYRK1A|MGC155217 \\
\hline XP_592833.3 & XM_592833.3 & - \\
\hline NP_001093768.1 & NM_001100298.1 & - \\
\hline XP_609767.3 & XM_609767.3 & - \\
\hline XP_001250674.1 & XM_001250673.1 & - \\
\hline NP_001095392.1 & NM_001101922.1 & MGC152387 \\
\hline XP_870428.2 & XM_865335.2 & - \\
\hline XP_598392.3 & XM_598392.3 & - \\
\hline NP_001069025.1 & NM_001075557.1 & MGC139658 \\
\hline NP_001095662.1 & NM_001102192.1 & MGC159759 \\
\hline NP_001094780.1 & NM_001101310.1 & - \\
\hline NP_001103488.1 & NM_001110018.1 & ERK1 \\
\hline NP_786987.1 & NM_175793.2 & ERK2 \\
\hline XP_605323.3 & XM_605323.3 & - \\
\hline NP_001069388.1 & NM_001075920.1 & MGC152151 \\
\hline NP_001092550.1 & NM_001099080.1 & MGC148520 \\
\hline NP_001039575.1 & NM_001046110.1 & MGC138052 \\
\hline XP_869760.1 & XM_864667.2 & JNK1 \\
\hline NP_001039834.1 & NM_001046369.1 & MGC137545 \\
\hline NP_001077197.1 & NM_001083728.1 & - \\
\hline XP_886284.2 & XM_881191.2 & - \\
\hline NP_001095644.1 & NM_001102174.1 & MGC142910 \\
\hline NP_001073804.1 & NM_001080335.1 & MGC139500 \\
\hline NP_001014947.1 & NM_001014947.1 & - \\
\hline NP_001092423.1 & NM_001098953.1 & MGC160082 \\
\hline NP_001092357.1 & NM_001098887.1 & MGC157129 \\
\hline NP_001039880.2 & NM_001046415.2 & \\
\hline XP_873352.1 & XM_868259.2 & - \\
\hline NP_001076859.1 & NM_001083390.1 & MGC143354|MSSK1 \\
\hline XP_590178.3 & XM_590178.3 & - \\
\hline XP_590213.3 & XM_590213.3 & - \\
\hline
\end{tabular}




\begin{tabular}{|c|c|c|}
\hline NP_001033117.1 & NM_001038028.1 & MGC134472 \\
\hline NP_898907.2 & NM_183084.2 & MGC142782|STK12 \\
\hline XP_876025.1 & XM_870932.2 & - \\
\hline NP_001095481.1 & NM_001102011.1 & MGC148432 \\
\hline XP_617181.3 & XM_617181.3 & - \\
\hline NP_001092650.1 & NM_001099180.1 & MGC159543 \\
\hline XP_587386.3 & XM_587386.3 & - \\
\hline NP_001068858.1 & NM_001075390.1 & MGC139717 \\
\hline NP_001032545.1 & NM_001037468.1 & CDC7L1|MGC128732 \\
\hline NP_777060.2 & NM_174635.2 & MGC137728 \\
\hline NP_777061.1 & NM_174636.2 & - \\
\hline NP_001070012.1 & NM_001076544.1 & MGC137325 \\
\hline NP_776446.1 & NM_174021.2 & - \\
\hline NP_776778.1 & NM_174353.2 & - \\
\hline NP_001039810.1 & NM_001046345.1 & MGC137373 \\
\hline XP_612449.3 & XM_612449.3 & - \\
\hline XP_590874.2 & XM_590874.3 & C-MOS \\
\hline XP_611658.3 & XM_611658.3 & - \\
\hline NP_001039549.1 & NM_001046084.1 & MGC127293 \\
\hline NP_001039720.1 & NM_001046255.1 & MGC137500 \\
\hline XP_600615.3 & XM_600615.3 & - \\
\hline XP_871668.2 & XM_866575.2 & - \\
\hline NP_001039735.1 & NM_001046270.1 & MGC137163 \\
\hline NP_001077112.1 & NM_001083643.1 & MGC143098 \\
\hline NP_001095414.1 & NM_001101944.1 & MGC159441 \\
\hline XM_001788409.1 & XP_001788461.1 & \\
\hline NP_001092458.1 & NM_001098988.1 & MGC149008 \\
\hline XP_001249770.1 & XM_001249769.1 & - \\
\hline XP_610844.3 & XM_610844.3 & - \\
\hline XP_614489.3 & XM_614489.3 & - \\
\hline XP_871544.2 & XM_866451.2 & - \\
\hline XP_871937.2 & XM_866844.3 & \\
\hline XP_001256345.1 & XM_001256344.1 & - \\
\hline NP_001093171.1 & NM_001099701.1 & - \\
\hline NP_001107240.1 & NM_001113768.1 & - \\
\hline XP_587377.2 & XM_587377.3 & - \\
\hline XP_001253752.1 & XM_001253751.1 & - \\
\hline XP_601098.3 & XM_601098.3 & - \\
\hline NP_001070357.1 & NM_001076889.1 & MGC139974 \\
\hline XP_001256461.1 & XM_001256460.1 & - \\
\hline XP_582577.2 & XM_582577.3 & - \\
\hline NP_001091634.1 & NM_001098165.1 & - \\
\hline XP_871637.2 & XM_866544.2 & - \\
\hline
\end{tabular}




\begin{tabular}{|c|c|c|}
\hline NP_001091496.1 & NM_001098027.1 & - \\
\hline XP_001250240.1 & XM_001250239.1 & - \\
\hline XP_002700787.1 & XM_002700741.1 & - \\
\hline NP_001019995.1 & NM_001024824.1 & - \\
\hline NP_001091555.1 & NM_001098086.1 & - \\
\hline NP_001033142.1 & NM_001038053.1 & MGC134528 \\
\hline NP_835210.2 & NM_178109.2 & PKR|PRKR \\
\hline NP_001033262.1 & NM_001038173.1 & MGC133927 \\
\hline XP_587229.3 & XM_587229.3 & - \\
\hline NP_001068621.1 & NM_001075153.1 & MGC142543 \\
\hline NP_001076896.1 & NM_001083427.1 & PLK-4 \\
\hline XP_589317.3 & XM_589317.3 & - \\
\hline NP_001095491.1 & NM_001102021.1 & MGC165975 \\
\hline NP_001095999.1 & NM_001102529.1 & MGC138084 \\
\hline XP_001787951.1 & XM_001787899.1 & \\
\hline NP_001092602.1 & NM_001099132.1 & MGC139332 \\
\hline XP_607080.3 & XM_607080.3 & - \\
\hline XP_617685.3 & XM_617685.3 & - \\
\hline XP_614568.3 & XM_614568.3 & - \\
\hline NP_001095648.1 & NM_001102178.1 & MGC165897 \\
\hline XP_603264.3 & XM_603264.3 & - \\
\hline XP_585177.3 & XM_585177.3 & - \\
\hline XP_604678.3 & XM_604678.3 & - \\
\hline NP_001095791.1 & NM_001102321.1 & \\
\hline NP_001039399.2 & NM_001045934.2 & \\
\hline XP_609757.3 & XM_609757.3 & - \\
\hline NP_001093784.1 & NM_001100314.1 & - \\
\hline XP_600897.2 & XM_600897.3 & - \\
\hline NP_001094675.1 & NM_001101205.1 & - \\
\hline XP_598279.3 & XM_598279.3 & - \\
\hline XP_582882.3 & XM_582882.3 & - \\
\hline XP_582977.3 & XM_582977.3 & - \\
\hline XP_612806.5 & XM_612806.3 & - \\
\hline XP_612318.2 & XM_612318.3 & - \\
\hline NP_776551.1 & NM_174126.2 & - \\
\hline NP_776973.1 & NM_174548.2 & - \\
\hline NP_776974.1 & NM_174549.2 & - \\
\hline NP_776972.1 & NM_174547.2 & - \\
\hline XP_602275.3 & XM_602275.3 & - \\
\hline XP_580739.2 & XM_580739.3 & - \\
\hline XP_886022.2 & XM_880929.2 & - \\
\hline XP_589178.3 & XM_589178.3 & - \\
\hline NP_001137553.1 & NM_001144081.1 & ASK1 \\
\hline XP_882494.1 & XM_877401.2 & - \\
\hline XP_607781.3 & XM_607781.3 & - \\
\hline XP_583510.2 & XM_583510.2 & - \\
\hline NP_001069294.1 & NM_001075826.1 & - \\
\hline
\end{tabular}




\begin{tabular}{|c|c|c|}
\hline XP_874795.2 & XM_869702.2 & - \\
\hline NP_001069293.2 & NM_001075825.2 & - \\
\hline XP_001249611.1 & XM_001249610.1 & - \\
\hline \multirow[t]{2}{*}{ XP_584270.3 } & XM_584270.3 & - \\
\hline & & HGK \\
\hline XP_588438.3 & XM_588438.3 & - \\
\hline NP_001092846.1 & NM_001099376.1 & MINK \\
\hline XP_001251137.1 & XM_001251136.1 & - \\
\hline NP_001015602.1 & NM_001015602.1 & - \\
\hline XP_001250083.1 & XM_001250082.1 & - \\
\hline XP_615938.3 & XM_615938.3 & - \\
\hline XP_616244.3 & XM_616244.3 & - \\
\hline NP_001070366.1 & NM_001076898.1 & MGC139676 \\
\hline XP_591400.3 & XM_591400.3 & - \\
\hline XP_001252146.1 & XM_001252145.1 & - \\
\hline NP_001069652.1 & NM_001076184.1 & MGC138047 \\
\hline XP_591106.3 & XM_591106.3 & - \\
\hline XP_585734.1 & XM_585734.3 & - \\
\hline XP_604746.3 & XM_604746.3 & - \\
\hline XP_001252985.1 & XM_001252984.1 & - \\
\hline NP_001015603.1 & NM_001015603.1 & - \\
\hline XP_582418.3 & XM_582418.3 & - \\
\hline XP_616664.3 & XM_616664.3 & - \\
\hline XP_872869.2 & XM_867776.2 & - \\
\hline XP_614451.3 & XM_614451.3 & - \\
\hline XP_610976.3 & XM_610976.3 & - \\
\hline XP_587191.3 & XM_587191.3 & - \\
\hline NP_899666.1 & NM_183407.2 & MGC128993 \\
\hline XP_612526.3 & XM_612526.3 & - \\
\hline NP_001033160.2 & NM_001038071.2 & MGC134398|MGC140486 \\
\hline NP_001077162.1 & NM_001083693.1 & MGC151903 \\
\hline NP_001092508.1 & NM_001099038.1 & MGC140244 \\
\hline XP_002700545.1 & XM_002700499.1 & \\
\hline NP_001029217.1 & NM_001034045.1 & - \\
\hline XP_001254724.1 & XM_001254723.3 & - \\
\hline NP_001092541.1 & NM_001099071.1 & MGC159463 \\
\hline XP_585134.3 & XM_585134.3 & - \\
\hline XP_613548.2 & XM_613548.3 & - \\
\hline XP_001253285.1 & XM_001253284.1 & ABLL \\
\hline NP_776310.2 & NM_173885.2 & ACK|MGC137695 \\
\hline XP_881345.1 & XM_876252.2 & - \\
\hline XP_616782.3 & XM_616782.3 & - \\
\hline XP_594754.3 & XM_594754.3 & - \\
\hline XP_580552.3 & XM_580552.3 & - \\
\hline XP_001253887.1 & XM_001253886.1 & - \\
\hline XP_869603.2 & XM_864510.2 & - \\
\hline NP_001068865.1 & NM_001075397.1 & MGC142896 \\
\hline NP_001030183.1 & NM_001035011.1 & - \\
\hline NP_001069480.2 & NM_001076012.2 & - \\
\hline NP_001077189.1 & NM_001083720.1 & MGC140218 \\
\hline
\end{tabular}




\begin{tabular}{|c|c|c|}
\hline XP_592211.3 & XM_592211.3 & - \\
\hline NP_001096575.1 & NM_001103105.1 & MGC143211 \\
\hline XP_002703751.1 & XM_002703705.1 & \\
\hline XP_604305.2 & XM_604305.3 & 525946 \\
\hline XP_594054.3 & XM_594054.3 & - \\
\hline XP_590380.3 & XM_590380.3 & - \\
\hline XP_618140.3 & XM_618140.3 & - \\
\hline NP_001076910.1 & NM_001083441.1 & \\
\hline XP_618422.3 & XM_618422.3 & - \\
\hline XP_001788053.1 & XM_001788001.2 & - \\
\hline XP_611161.3 & XM_611161.3 & - \\
\hline XP_595537.3 & XM_595537.3 & - \\
\hline XP_614602.3 & XM_614602.3 & - \\
\hline XP_875687.2 & XM_870594.2 & - \\
\hline NP_001178427.1 & NM_001191498.1 & \\
\hline XP_613645.2 & XM_613645.3 & - \\
\hline XP_874493.2 & XM_869400.2 & - \\
\hline NP_001091536.2 & NM_001098067.1 & \\
\hline NP_001068718.2 & NM_001075250.2 & FADK \\
\hline NP_001095722.1 & NM_001102252.1 & MGC139227 \\
\hline NP_001178265.1 & NM_001191336.1 & - \\
\hline NP_001027471.1 & NM_001032300.1 & - \\
\hline NP_001103677.1 & NM_001110207.1 & MGC151817 \\
\hline XP_885574.2 & XM_880481.2 & FGFR \\
\hline NP_776743.1 & NM_174318.3 & - \\
\hline XP_602166.3 & XM_602166.3 & - \\
\hline XP_606794.3 & XM_606794.3 & - \\
\hline XP_590552.2 & XM_590552.3 & ir-A \\
\hline XP_001254386.1 & XM_001254385.1 & - \\
\hline XP_001790676.2 & XM_001790624.2 & \\
\hline XP_002689649.1 & XM_002689603.1 & - \\
\hline XP_002704759.1 & XM_002704713.1 & \\
\hline NP_001107236.1 & NM_001113764.1 & - \\
\hline XP_588863.3 & XM_588863.3 & - \\
\hline XP_589779.3 & XM_589779.3 & - \\
\hline NP_001013017.2 & NM_001012999.2 & HGFR|MGC166072|c-met \\
\hline XP_603857.3 & XM_603857.3 & - \\
\hline XP_591182.3 & XM_591182.3 & - \\
\hline XP_590263.3 & XM_590263.3 & - \\
\hline XP_612028.3 & XM_612028.3 & c-kit \\
\hline XP_590921.3 & XM_590921.3 & - \\
\hline NP_001069364.2 & NM_001075896.2 & \\
\hline XP_002703399.1 & XM_002703353.1 & \\
\hline XP_001789312.2 & XM_001789260.2 & - \\
\hline NP_001098934.2 & NM_001105464.2 & \\
\hline XP_001249767.1 & XM_001249766.1 & - \\
\hline
\end{tabular}




\begin{tabular}{|c|c|c|}
\hline NP_001069436.1 & NM_001075968.1 & MGC142356 \\
\hline XP_608273.2 & XM_608273.2 & - \\
\hline NP_001092461.1 & NM_001098991.1 & MGC159776 \\
\hline XP_586141.3 & XM_586141.3 & - \\
\hline NP_001071440.1 & NM_001077972.1 & - \\
\hline NP_001103664.1 & NM_001110194.1 & - \\
\hline NP_001029506.1 & NM_001034334.1 & MGC126900 \\
\hline XP_614963.2 & XM_614963.3 & - \\
\hline NP_001104274.1 & NM_001110804.1 & - \\
\hline XP_600086.3 & XM_600086.3 & - \\
\hline NP_001094530.1 & NM_001101060.1 & - \\
\hline NP_001032542.1 & NM_001037465.1 & MGC127145 \\
\hline XP_870655.1 & XM_865562.2 & - \\
\hline NP_001179636.1 & NM_001192707.1 & - \\
\hline NP_001029761.1 & NM_001034589.1 & MGC126902 \\
\hline NP_001098858.1 & NM_001105388.1 & MGC165932 \\
\hline XP_580898.3 & XM_580898.3 & - \\
\hline XP_870050.2 & XM_864957.2 & - \\
\hline NP_776390.1 & NM_173965.2 & TIE \\
\hline NP_776389.1 & NM_173964.2 & MGC139569 \\
\hline XP_591681.3 & XM_591681.3 & SYTK1 \\
\hline XP_613650.2 & XM_613650.3 & TRKA \\
\hline XP_002696558.1 & XM_002696512.1 & - \\
\hline NP_001178061.1 & NM_001191132.1 & VEGFR1 \\
\hline NP_001178273.1 & NM_001191344.1 & \\
\hline NP_001103470.1 & NM_001110000.1 & VEGFR2 |flk-1 \\
\hline NP_001035645.1 & NM_001040555.1 & - \\
\hline NP_001069164.1 & NM_001075696.1 & IRAK-2 \\
\hline XP_587469.2 & XM_587469.3 & - \\
\hline NP_001069466.1 & NM_001075998.1 & IRAK-4|MGC137816 \\
\hline XP_002698230.1 & XM_002698184.1 & \\
\hline NP_001033187.1 & NM_001038098.1 & MGC134164 \\
\hline XP_606040.2 & XM_606040.3 & - \\
\hline XP_590674.3 & XM_590674.3 & - \\
\hline XP_591774.3 & XM_591774.3 & - \\
\hline XP_615760.3 & XM_615760.3 & - \\
\hline XP_001250856.1 & XM_001250855.1 & MGC152244 \\
\hline NP_001095323.1 & NM_001101853.1 & MGC179376 \\
\hline XP_002691037.1 & XM_002690991.1 & - \\
\hline XP_001249388.1 & XM_001249387.1 & - \\
\hline NP_001095461.1 & NM_001101991.1 & MGC165851 \\
\hline XP_873070.2 & XM_867977.2 & - \\
\hline XP_870499.1 & XM_865406.2 & - \\
\hline NP_001075064.1 & NM_001081595.1 & MGC154981 \\
\hline NP_001014964.1 & NM_001014964.1 & MGC128775 \\
\hline XP_002687048.1 & XM_002687002.1 & - \\
\hline XP_001788801.1 & XM_001788749.1 & \\
\hline
\end{tabular}




\begin{tabular}{|c|c|c|}
\hline XP_874651.2 & XM_869558.2 & - \\
\hline NP_001095975.1 & NM_001102505.1 & MGC165882 \\
\hline NP_001093797.1 & NM_001100327.1 & MGC127532 \\
\hline NP_001030184.1 & NM_001035012.1 & MGC127456 \\
\hline NP_001029782.1 & NM_001034610.1 & MGC152488 \\
\hline NP_001095354.1 & NM_001101884.1 & MGC139457 \\
\hline & & ActR-IA \\
\hline NP_001076948.1 & NM_001083479.1 & - \\
\hline NP_788836.2 & NM_176663.3 & - \\
\hline XP_586402.3 & XM_586402.3 & BMPR-IA \\
\hline XP_616508.3 & XM_616508.3 & - \\
\hline NP_001070268.1 & NM_001076800.1 & ACVR2 \\
\hline NP_001098798.1 & NM_001105328.1 & ALK6|BMP15|BMPR-IB|BMPRIB \\
\hline NP_777046.1 & NM_174621.2 & ActR-IIB|ActRIIB \\
\hline NP_776652.1 & NM_174227.3 & bmprii \\
\hline NP_776920.1 & NM_174495.2 & \\
\hline XP_617592.3 & XM_617592.3 & - \\
\hline & & MGC129022 \\
\hline XP_615445.2 & XM_615445.3 & - \\
\hline NP_001029865.1 & NM_001034693.1 & \\
\hline XP_001256760.1 & XM_001256759.1 & \\
\hline & & \\
\hline
\end{tabular}




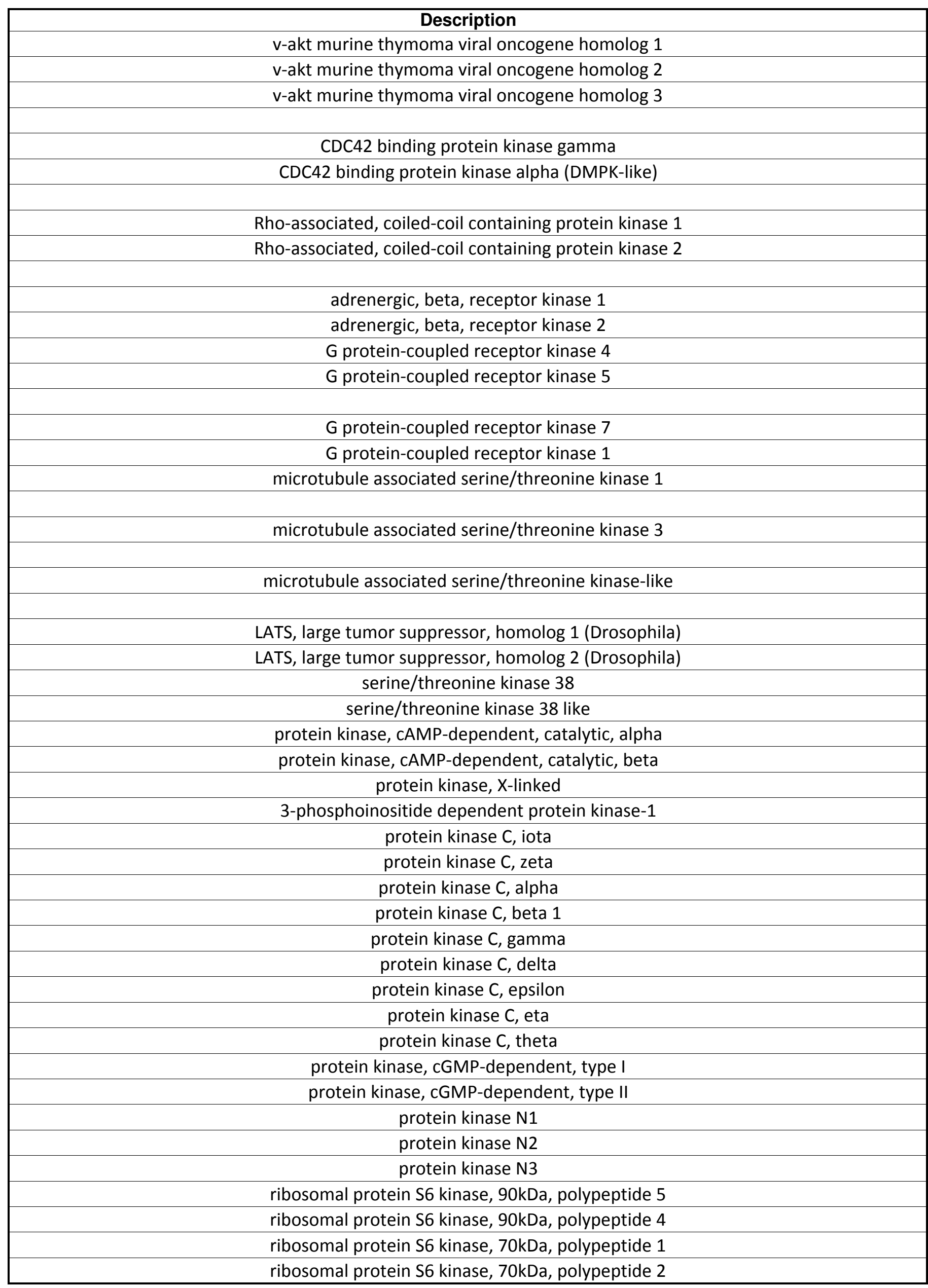


ribosomal protein $\mathrm{S} 6$ kinase, $90 \mathrm{kDa}$, polypeptide 2

ribosomal protein $\mathrm{S} 6$ kinase, $90 \mathrm{kDa}$, polypeptide 3

ribosomal protein $\mathrm{S} 6$ kinase, $90 \mathrm{kDa}$, polypeptide 1

ribosomal protein $\mathrm{S} 6$ kinase, $90 \mathrm{kDa}$, polypeptide 6

ribosomal protein $\mathrm{S} 6$ kinase, $52 \mathrm{kDa}$, polypeptide 1

ribosomal protein $\mathrm{S} 6$ kinase-like 1

similar to Putative serine/threonine-protein kinase F31E3.2

serum/glucocorticoid regulated kinase 1

serum/glucocorticoid regulated kinase 2

serum/glucocorticoid regulated kinase family, member 3

serine/threonine kinase $32 \mathrm{~A}$

serine/threonine kinase 32B

serine/threonine kinase $32 \mathrm{C}$

twinfilin, actin-binding protein, homolog 1 (Drosophila)

twinfilin, actin-binding protein, homolog 2 (Drosophila)

chaperone, $A B C 1$ activity of bc1 complex homolog (S. pombe)

aarF domain containing kinase 4

aarF domain containing kinase 1

aarF domain containing kinase 5

aarF domain containing kinase 2

transient receptor potential cation channel, subfamily $\mathrm{M}$, member 7

transient receptor potential cation channel, subfamily $M$, member 6

eukaryotic elongation factor-2 kinase

alpha-kinase 2

alpha-kinase 1

breakpoint cluster region

bromodomain containing 2

bromodomain containing 3

bromodomain containing 4

bromodomain, testis-specific

Fas-activated serine/threonine kinase

serine/threonine kinase 19

heat shock $22 \mathrm{kDa}$ protein 8

branched chain ketoacid dehydrogenase kinase

pyruvate dehydrogenase kinase, isozyme 1

pyruvate dehydrogenase kinase, isozyme 2

pyruvate dehydrogenase kinase, isozyme 3

pyruvate dehydrogenase kinase, isozyme 4

ataxia telangiectasia mutated

ataxia telangiectasia and Rad3 related

protein kinase, DNA-activated, catalytic polypeptide

mechanistic target of rapamycin (serine/threonine kinase)

SMG1 homolog, phosphatidylinositol 3-kinase-related kinase (C. elegans)

transformation/transcription domain-associated protein

RIO kinase 1 (yeast)

RIO kinase 2 (yeast)

RIO kinase 3 (yeast)

TAF1 RNA polymerase II, TATA box binding protein (TBP)-associated factor, 250kDa

tripartite motif-containing 33 
tripartite motif-containing 24

calcium/calmodulin-dependent protein kinase I

pregnancy upregulated non-ubiquitously expressed CaM kinase

similar to Calcium/calmodulin-dependent protein kinase ID

calcium/calmodulin-dependent protein kinase IG

calcium/calmodulin-dependent protein kinase (CaM kinase) II alpha

calcium/calmodulin-dependent protein kinase (CaM kinase) II beta

calcium/calmodulin-dependent protein kinase (CaM kinase) II delta

calcium/calmodulin-dependent protein kinase (CaM kinase) II gamma

similar to Calcium/calmodulin-dependent protein kinase type IV (CAM kinase-GR) (CaMK IV)

protein kinase, AMP-activated, alpha 1 catalytic subunit

protein kinase, AMP-activated, alpha 2 catalytic subunit

BR serine/threonine kinase 1

BR serine/threonine kinase 2

CHK1 checkpoint homolog (S. pombe)

hormonally upregulated Neu-associated kinase

serine/threonine kinase 11

$\mathrm{MAP} /$ microtubule affinity-regulating kinase 1

$\mathrm{MAP} /$ microtubule affinity-regulating kinase 2

$\mathrm{MAP} /$ microtubule affinity-regulating kinase 3

$\mathrm{MAP} /$ microtubule affinity-regulating kinase 4

hypothetical LOC618482

maternal embryonic leucine zipper kinase

hypothetical LOC530210

NUAK family, SNF1-like kinase, 1

NUAK family, SNF1-like kinase, 2

PAS domain containing serine/threonine kinase

SNF1-like kinase 2

similar to KIAA0999 protein

SNF related kinase

serine/threonine kinase 40

serine/threonine kinase 33

CaM kinase-like vesicle-associated

calcium/calmodulin-dependent serine protein kinase (MAGUK family)

death-associated protein kinase 1

death-associated protein kinase 2

death-associated protein kinase 3

serine/threonine kinase $17 \mathrm{a}$

serine/threonine kinase $17 \mathrm{~b}$

doublecortin-like kinase 2

doublecortin-like kinase 3

mitogen-activated protein kinase-activated protein kinase 2

mitogen-activated protein kinase-activated protein kinase 3

mitogen-activated protein kinase-activated protein kinase 5

MAP kinase interacting serine/threonine kinase 1

MAP kinase interacting serine/threonine kinase 2

myosin light chain kinase 3 


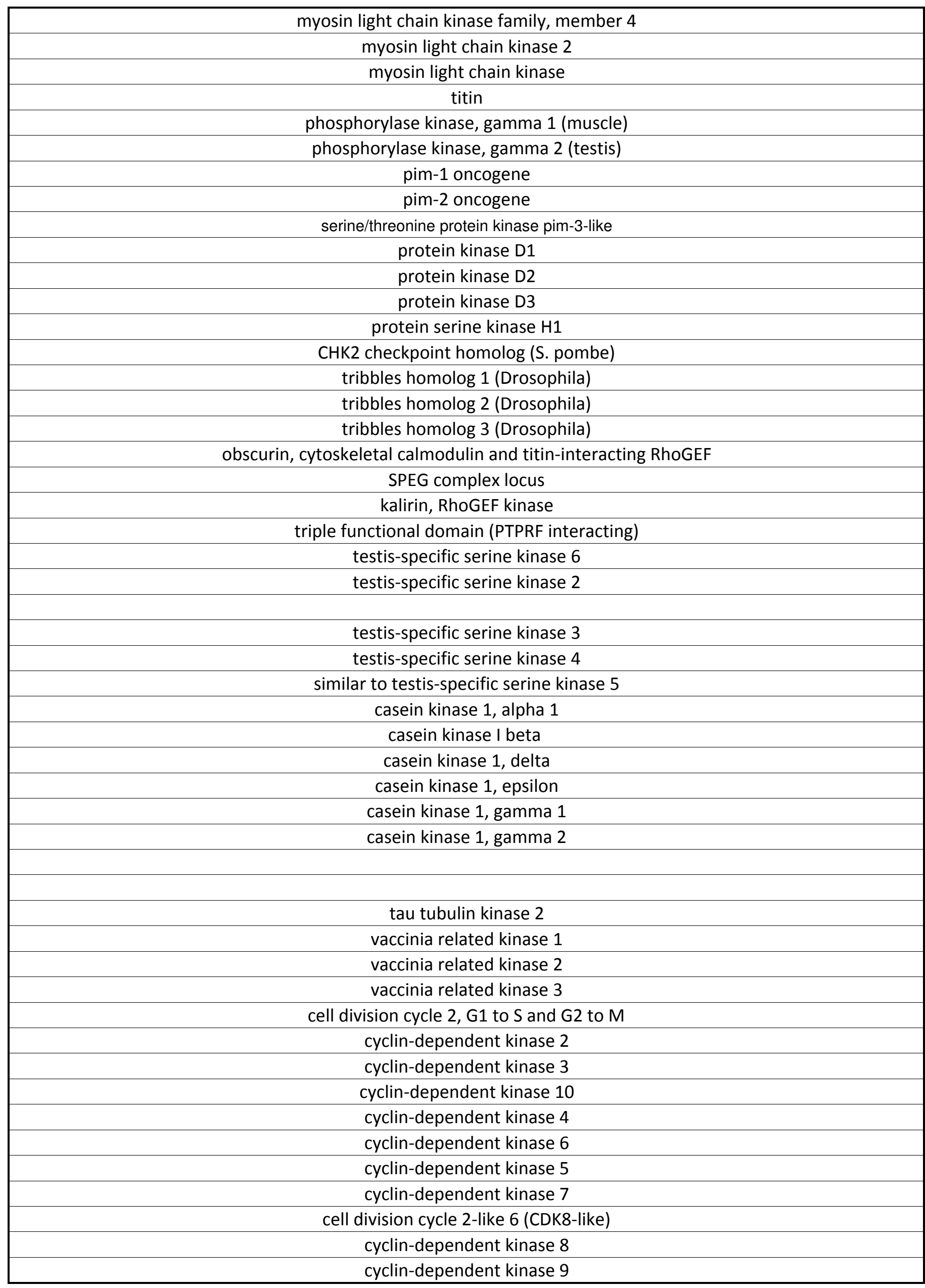


cell division cycle 2-like 5 (cholinesterase-related cell division controller)

Cdc2-related kinase, arginine/serine-rich

cell division cycle 2-like 1 (PITSLRE proteins)

PCTAIRE protein kinase 1

PCTAIRE protein kinase 2

PCTAIRE protein kinase 3

cyclin-dependent kinase 14

cyclin-dependent kinase 15

cell cycle related kinase

cyclin-dependent kinase-like 1 (CDC2-related kinase)

cyclin-dependent kinase-like 2 (CDC2-related kinase)

cyclin-dependent kinase-like 3

cyclin-dependent kinase-like 4

CDC-like kinase 1

CDC-like kinase 2

CDC-like kinase 3

CDC-like kinase 4

dual-specificity tyrosine-(Y)-phosphorylation regulated kinase $1 \mathrm{~A}$

dual-specificity tyrosine-(Y)-phosphorylation regulated kinase 1B

similar to Dual-specificity tyrosine-(Y)-phosphorylation regulated kinase 2

ual-specificity tyrosine-(Y)-phosphorylation regulated kinase 3

hypothetical LOC531276

homeodomain interacting protein kinase 1

homeodomain interacting protein kinase 2

homeodomain interacting protein kinase 3

homeodomain interacting protein kinase 4

PRP4 pre-mRNA processing factor 4 homolog $B$ (yeast)

glycogen synthase kinase 3 alpha

glycogen synthase kinase 3 beta

mitogen-activated protein kinase 3

mitogen-activated protein kinase 1

mitogen-activated protein kinase 6

mitogen-activated protein kinase 4

mitogen-activated protein kinase 7

mitogen-activated protein kinase 15

mitogen-activated protein kinase 8

mitogen-activated protein kinase 9

mitogen-activated protein kinase 10

nemo-like kinase

mitogen-activated protein kinase 14

mitogen-activated protein kinase 11

mitogen-activated protein kinase 13

mitogen-activated protein kinase 12

intestinal cell (MAK-like) kinase

male germ cell-associated kinase

renal tumor antigen

SFRS protein kinase 3

SFRS protein kinase 1

SFRS protein kinase 2 
aurora kinase $A$

aurora kinase $B$

aurora kinase $\mathrm{C}$

BUB1 budding uninhibited by benzimidazoles 1 homolog (yeast)

BUB1 budding uninhibited by benzimidazoles 1 homolog beta (yeast)

TP53 regulating kinase

calcium/calmodulin-dependent protein kinase kinase 1, alpha

calcium/calmodulin-dependent protein kinase kinase 2, beta

cell division cycle 7 homolog (S. cerevisiae)

casein kinase 2, alpha 1 polypeptide

casein kinase 2 , alpha prime polypeptide

germ cell associated 2 (haspin)

conserved helix-loop-helix ubiquitous kinase

inhibitor of kappa light polypeptide gene enhancer in B-cells, kinase beta

inhibitor of kappa light polypeptide gene enhancer in B-cells, kinase epsilon

TANK-binding kinase 1

v-mos Moloney murine sarcoma viral oncogene homolog

AP2 associated kinase 1

cyclin $\mathrm{G}$ associated kinase

serine/threonine kinase 16

NIMA (never in mitosis gene a)- related kinase 10

NIMA (never in mitosis gene a)- related kinase 11

NIMA (never in mitosis gene a)-related kinase 2

NIMA (never in mitosis gene a)-related kinase 3

NIMA (never in mitosis gene a)-related kinase 4

NIMA (never in mitosis gene a)-related kinase 5

NIMA (never in mitosis gene a)-related kinase 6

NIMA (never in mitosis gene a)-related kinase 7

NIMA (never in mitosis gene a)- related kinase 8

NIMA (never in mitosis gene a)- related kinase 9

similar to SH3-binding kinase

SH3-binding domain kinase family, member 2

similar to protein kinase

PTEN induced putative kinase 1

serine/threonine kinase 35

PDLIM1 interacting kinase 1 like

similar to Gene model 1082, (NCBI)

testis expressed 14

nuclear receptor binding protein 1

nuclear receptor binding protein 2

U2AF homology motif (UHM) kinase 1

ribonuclease $L$ (2',5'-oligoisoadenylate synthetase-dependent)

hypothetical LOC614899 


\begin{tabular}{|c|}
\hline hypothetical LOC514490 \\
\hline serine/threonine kinase 31 \\
\hline protein kinase domain containing, cytoplasmic homolog (mouse) \\
\hline receptor interacting protein kinase 5 \\
\hline eukaryotic translation initiation factor 2-alpha kinase 3 \\
\hline eukaryotic translation initiation factor 2-alpha kinase 1 \\
\hline eukaryotic translation initiation factor 2 -alpha kinase 2 \\
\hline polo-like kinase 1 (Drosophila) \\
\hline polo-like kinase 2 (Drosophila) \\
\hline polo-like kinase 3 (Drosophila) \\
\hline polo-like kinase 4 \\
\hline similar to Serine/threonine-protein kinase PLK5 (Polo-like kinase 5) (PLK-5) \\
\hline SCY1-like 1 (S. cerevisiae) \\
\hline SCY1-like 2 (S. cerevisiae) \\
\hline SCY1-like 3 (S. cerevisiae) \\
\hline PX domain containing serine/threonine kinase \\
\hline TBC1 domain containing kinase \\
\hline tousled-like kinase 1 \\
\hline tousled-like kinase 2 \\
\hline PDZ binding kinase \\
\hline TTK protein kinase \\
\hline serine/threonine kinase 36 , fused homolog (Drosophila) \\
\hline unc-51-like kinase 1 (C. elegans) \\
\hline unc-51-like kinase 2 (C. elegans) \\
\hline unc-51-like kinase 3 (C. elegans) \\
\hline unc-51-like kinase 4 (C. elegans) \\
\hline phosphoinositide-3-kinase, regulatory subunit 4 \\
\hline protein kinase, membrane associated tyrosine/threonine 1 \\
\hline WEE1 homolog (S. pombe) \\
\hline WEE1 homolog 2 (S. pombe) \\
\hline WNK lysine deficient protein kinase 1 \\
\hline WNK lysine deficient protein kinase 2 \\
\hline WNK lysine deficient protein kinase 3 \\
\hline natriuretic peptide receptor $\mathrm{A} /$ guanylate cyclase $\mathrm{A}$ (atrionatriuretic peptide receptor $\mathrm{A}$ ) \\
\hline natriuretic peptide receptor $\mathrm{B} /$ guanylate cyclase $\mathrm{B}$ (atrionatriuretic peptide receptor $\mathrm{B}$ ) \\
\hline guanylate cyclase 2D, membrane (retina-specific) \\
\hline guanylate cyclase $2 \mathrm{~F}$, retinal \\
\hline guanylate cyclase $2 \mathrm{C}$ (heat stable enterotoxin receptor) \\
\hline mitogen-activated protein kinase kinase kinase 1 \\
\hline mitogen-activated protein kinase kinase kinase 2 \\
\hline mitogen-activated protein kinase kinase kinase 3 \\
\hline mitogen-activated protein kinase kinase kinase 4 \\
\hline mitogen-activated protein kinase kinase kinase 5 \\
\hline mitogen-activated protein kinase kinase kinase 6 \\
\hline mitogen-activated protein kinase kinase kinase 15 \\
\hline yeast Sps1/Ste20-related kinase 4 (S. cerevisiae) \\
\hline serine threonine kinase 39 (STE20/SPS1 homolog, yeast) \\
\hline
\end{tabular}


mitogen-activated protein kinase kinase kinase kinase 2

mitogen-activated protein kinase kinase kinase kinase 1

mitogen-activated protein kinase kinase kinase kinase 5

mitogen-activated protein kinase kinase kinase kinase 3

TRAF2 and NCK interacting kinase

misshapen-like kinase 1 (zebrafish)

Nik related kinase

serine/threonine kinase 4

serine/threonine kinase 3 (STE20 homolog, yeast)

myosin IIIA

myosin IIIB

p21/Cdc42/Rac1-activated kinase 1 (STE20 homolog, yeast)

similar to serine/threonine kinase

p21 (CDKN1A)-activated kinase 3

p21(CDKN1A)-activated kinase 4

p21(CDKN1A)-activated kinase 7

p21(CDKN1A)-activated kinase 6

serine/threonine kinase 10

STE20-like kinase (yeast)

protein kinase LYK5

STE20-related kinase adaptor beta

TAO kinase 1

TAO kinase 2

TAO kinase 3

serine/threonine kinase 24 (STE20 homolog, yeast)-like

serine/threonine protein kinase MST4

serine/threonine kinase 25 (STE20 homolog, yeast)

mitogen-activated protein kinase kinase 1

mitogen-activated protein kinase kinase 2

mitogen-activated protein kinase kinase 3

mitogen-activated protein kinase kinase 4

mitogen-activated protein kinase kinase 5

mitogen-activated protein kinase kinase 6

mitogen-activated protein kinase kinase 7

mitogen-activated protein kinase kinase kinase 8

mitogen-activated protein kinase kinase kinase 14

c-abl oncogene 1 , receptor tyrosine kinase

v-abl Abelson murine leukemia viral oncogene homolog 2 (arg, Abelson-related gene)

activated p21cdc42Hs kinase

tyrosine kinase, non-receptor, 1

anaplastic lymphoma receptor tyrosine kinase

AXL receptor tyrosine kinase

c-mer proto-oncogene tyrosine kinase

TYRO3 protein tyrosine kinase

PTK7 protein tyrosine kinase 7

c-src tyrosine kinase

megakaryocyte-associated tyrosine kinase

discoidin domain receptor tyrosine kinase 1

discoidin domain receptor tyrosine kinase 2 


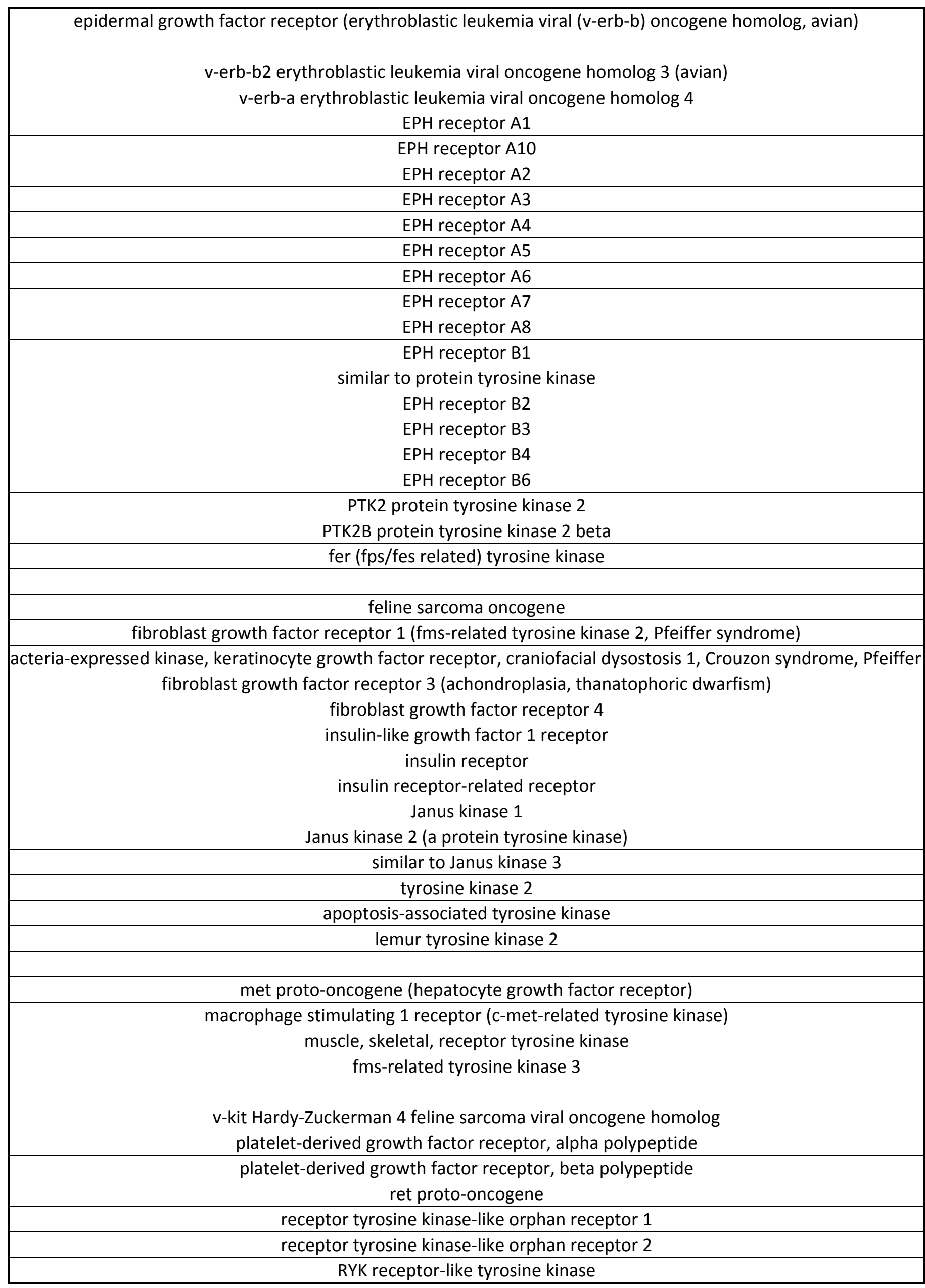




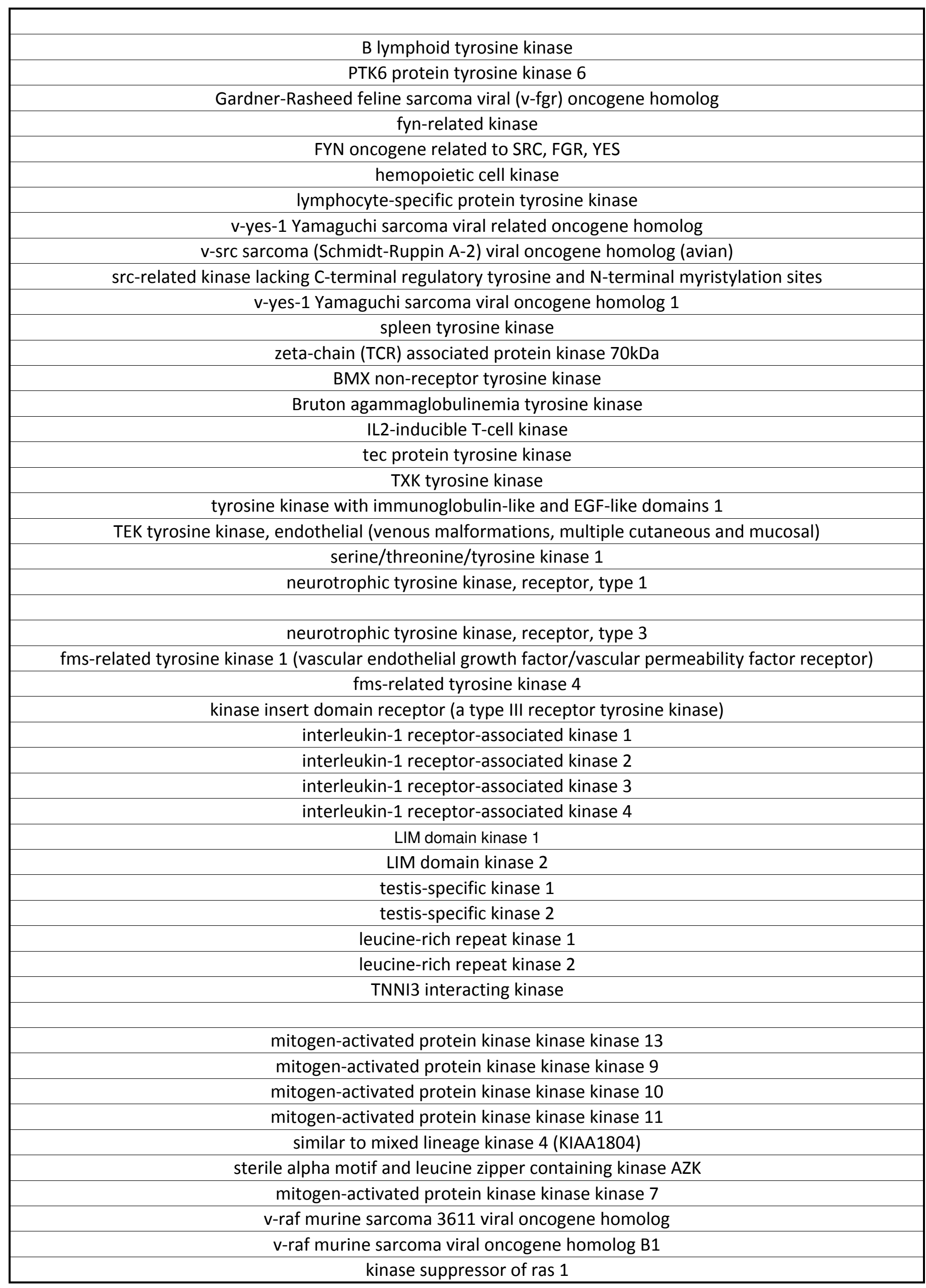




\begin{tabular}{|c|}
\hline kinase suppressor of ras 2 \\
\hline v-raf-1 murine leukemia viral oncogene homolog 1 \\
\hline receptor-interacting serine-threonine kinase 4 \\
\hline receptor (TNFRSF)-interacting serine-threonine kinase 1 \\
\hline receptor-interacting serine-threonine kinase 2 \\
\hline receptor-interacting serine-threonine kinase 3 \\
\hline activin A receptor type II-like 1 \\
\hline activin A receptor, type I \\
\hline activin A receptor, type IB \\
\hline activin A receptor, type IC \\
\hline bone morphogenetic protein receptor, type IA \\
\hline bone morphogenetic protein receptor, type IB \\
\hline activin A receptor, type IIA \\
\hline activin A receptor, type IIB \\
\hline transforming growth factor, beta receptor I (activin A receptor type II-like kinase, 53kDa) \\
\hline transforming growth factor, beta receptor II (70/80kDa) \\
\hline integrin-linked kinase \\
\hline mixed lineage kinase domain-like \\
\hline \\
\hline
\end{tabular}




\begin{tabular}{|c|c|c|c|c|}
\hline Chr & cDNA lenght & ORF length & Protein size & kinase domain (KD)n size \\
\hline 21 & 1691 & 1443 & 480 & 259 \\
\hline 18 & 3460 & 1446 & 481 & 258 \\
\hline 16 & 1776 & 1440 & 479 & 258 \\
\hline 18 & 2434 & 1857 & 618 & 269 \\
\hline 29 & 5018 & 4632 & 1543 & 266 \\
\hline 16 & 5265 & 5265 & 1754 & 267 \\
\hline 21 & 6560 & 5271 & 1756 & 267 \\
\hline 24 & 4793 & 4104 & 1367 & 263 \\
\hline 11 & 5053 & 4167 & 1388 & 263 \\
\hline 17 & 5837 & NA & 1820 & 110 \\
\hline 29 & 2520 & 2070 & 689 & 263 \\
\hline 17 & 2791 & 2067 & 688 & 263 \\
\hline 6 & 1752 & 1752 & 583 & 263 \\
\hline 26 & 2539 & 1773 & 590 & 263 \\
\hline 7 & 2853 & 1725 & 574 & 259 \\
\hline 1 & 2227 & 1659 & 552 & 264 \\
\hline 12 & 2898 & 1686 & 561 & 266 \\
\hline 7 & 4719 & 4719 & 1572 & 274 \\
\hline 3 & 5414 & 5136 & 1711 & 274 \\
\hline 7 & 5651 & 4101 & 1366 & 274 \\
\hline 20 & 7677 & 7302 & 2433 & 274 \\
\hline 13 & 2736 & 2643 & 880 & 276 \\
\hline 3 & 795 & 795 & 264 & 161 \\
\hline 9 & 7537 & 3372 & 1123 & 306 \\
\hline 12 & 4733 & 3048 & 1015 & 305 \\
\hline 23 & 3545 & 1398 & 465 & 294 \\
\hline 5 & 4209 & 1395 & 464 & 294 \\
\hline 7 & 2522 & 1056 & 351 & 255 \\
\hline 3 & 1750 & 1056 & 351 & 255 \\
\hline$x$ & 1139 & 957 & 318 & 255 \\
\hline 25 & 1820 & 1605 & 534 & 265 \\
\hline 1 & 2813 & 1791 & 596 & 269 \\
\hline 16 & 2240 & 1785 & 594 & 267 \\
\hline 19 & 2324 & 2019 & 672 & 259 \\
\hline 25 & 2955 & 2022 & 673 & 259 \\
\hline 18 & 3032 & 2094 & 697 & 264 \\
\hline 22 & 3019 & 2031 & 676 & 255 \\
\hline 11 & 5491 & 2214 & 737 & 261 \\
\hline 10 & 2389 & 2049 & 682 & 260 \\
\hline 13 & 3181 & 2121 & 706 & 255 \\
\hline 26 & 2775 & 2016 & 671 & 260 \\
\hline 6 & 2289 & 2289 & 762 & 259 \\
\hline 7 & 3059 & 2835 & 944 & 260 \\
\hline 3 & 3226 & 2946 & 981 & 260 \\
\hline 11 & 3386 & 2661 & 886 & 260 \\
\hline 10 & 4103 & 2346 & 781 & 270 \\
\hline 29 & 3061 & 2316 & 771 & 269 \\
\hline 19 & 1585 & 1584 & 527 & 262 \\
\hline 29 & 1791 & 1560 & 519 & 262 \\
\hline
\end{tabular}




\begin{tabular}{|c|c|c|c|c|}
\hline 9 & 3605 & 2202 & 733 & 260 \\
\hline$x$ & 2927 & 2223 & 740 & 260 \\
\hline 2 & 3157 & 2208 & 735 & 260 \\
\hline$X$ & 2635 & 2235 & 744 & 258 \\
\hline 16 & 4415 & 3186 & 1061 & 232 \\
\hline 10 & 3608 & 1326 & 441 & 283 \\
\hline 19 & 1628 & 819 & 272 & 171 \\
\hline 9 & 2382 & 1296 & 431 & 258 \\
\hline 13 & 1755 & 1104 & 367 & 258 \\
\hline 14 & 3363 & 1473 & 490 & 258 \\
\hline 7 & 1852 & 1101 & 366 & 259 \\
\hline 6 & 1479 & 1479 & 492 & 261 \\
\hline 26 & 3409 & 2889 & 962 & 261 \\
\hline 5 & 1242 & 1053 & 350 & NA \\
\hline 22 & 1614 & 1050 & 349 & NA \\
\hline 16 & 2903 & 1947 & 648 & NA \\
\hline 18 & 2149 & 1578 & 525 & NA \\
\hline 10 & 2274 & 1572 & 523 & NA \\
\hline 14 & 1927 & 1743 & 580 & NA \\
\hline 4 & 2160 & 1878 & 625 & NA \\
\hline 10 & 7214 & 5601 & 1866 & NA \\
\hline 8 & 6756 & 6480 & 2159 & NA \\
\hline 25 & 5774 & 2172 & 723 & NA \\
\hline 21 & 5352 & NA & 1783 & NA \\
\hline 24 & 6916 & 6360 & 2119 & NA \\
\hline 6 & 4289 & 3774 & 1257 & NA \\
\hline 17 & 3127 & 2967 & 988 & NA \\
\hline 23 & 2412 & 2412 & 803 & NA \\
\hline 11 & 3093 & 2169 & 722 & NA \\
\hline 7 & 5608 & 4107 & 1368 & NA \\
\hline 3 & 2936 & 2808 & 935 & NA \\
\hline 4 & 1829 & 1632 & 543 & NA \\
\hline 23 & 2416 & 510 & 169 & NA \\
\hline 17 & 1906 & 591 & 196 & NA \\
\hline 25 & 1872 & 1239 & 412 & NA \\
\hline 2 & 3859 & 1317 & 438 & NA \\
\hline 19 & 2277 & 1032 & 343 & NA \\
\hline$x$ & 2082 & 1248 & 415 & NA \\
\hline 4 & 3805 & 1224 & 407 & NA \\
\hline 15 & 9406 & 9165 & 3054 & NA \\
\hline 1 & 8268 & 7935 & 2644 & NA \\
\hline 14 & 12953 & 12375 & 4124 & NA \\
\hline 16 & 8303 & 7656 & 2551 & NA \\
\hline 25 & 15784 & 10977 & 3658 & NA \\
\hline 25 & 12190 & 11496 & 3831 & NA \\
\hline 23 & 1952 & NA & 544 & NA \\
\hline 7 & 1871 & 1656 & 551 & NA \\
\hline 24 & 4335 & 1560 & 519 & NA \\
\hline$x$ & 7765 & 6090 & 2029 & NA \\
\hline 3 & 3622 & 3330 & 1109 & NA \\
\hline
\end{tabular}




\begin{tabular}{|c|c|c|c|c|}
\hline 18 & 2934 & 2202 & 733 & NA \\
\hline 4 & 3365 & 2808 & 935 & NA \\
\hline 22 & 1456 & 1113 & 370 & 257 \\
\hline$X$ & 1410 & 1029 & 342 & 256 \\
\hline 13 & 1510 & 1140 & 379 & 272 \\
\hline 16 & 2357 & 1425 & 474 & 255 \\
\hline 7 & 3032 & 1437 & 478 & 259 \\
\hline 4 & 1912 & 1629 & 542 & 259 \\
\hline 6 & 1537 & 1500 & 499 & 259 \\
\hline 28 & 1780 & 1488 & 495 & 259 \\
\hline 10 & 1532 & 1392 & 463 & 255 \\
\hline 20 & 1698 & 1698 & 565 & 268 \\
\hline 3 & 2153 & 1659 & 552 & 253 \\
\hline 18 & 3018 & 2481 & 826 & 252 \\
\hline 29 & 2175 & 2175 & 724 & 265 \\
\hline 29 & 2276 & 1431 & 476 & 257 \\
\hline 1 & 2028 & 2028 & 675 & 261 \\
\hline 7 & 1623 & 1326 & 441 & 264 \\
\hline 16 & 2681 & 2388 & 795 & 252 \\
\hline 29 & 2274 & 2274 & 757 & 252 \\
\hline 21 & 2995 & 2379 & 792 & 252 \\
\hline 18 & 1947 & 1329 & 442 & 252 \\
\hline 14 & 1518 & 1518 & 505 & 250 \\
\hline 8 & 2445 & 1953 & 650 & 253 \\
\hline 20 & 2122 & 1311 & 436 & 252 \\
\hline 5 & 2227 & 1989 & 662 & 252 \\
\hline 16 & 1476 & 1476 & 491 & 251 \\
\hline 3 & 4495 & 4128 & 1375 & 253 \\
\hline 15 & 3639 & 2721 & 906 & 252 \\
\hline 15 & 6891 & 3945 & 1314 & 252 \\
\hline 1 & 1797 & NA & 599 & 255 \\
\hline 22 & 5000 & 2085 & 694 & 254 \\
\hline 3 & 1749 & 1311 & 436 & 195 \\
\hline 15 & 2228 & 1461 & 486 & 266 \\
\hline 22 & 2932 & 1515 & 504 & 263 \\
\hline$X$ & 3302 & 2727 & 908 & 265 \\
\hline 8 & 2519 & 4293 & 1430 & 263 \\
\hline 10 & 1579 & 1452 & 483 & 263 \\
\hline 7 & 3120 & 1365 & 454 & 263 \\
\hline 4 & 1760 & 1245 & 414 & 261 \\
\hline 2 & 1577 & 1119 & 372 & 260 \\
\hline 12 & 1296 & NA & 431 & 258 \\
\hline 17 & 2304 & 2304 & 767 & 258 \\
\hline 22 & 2607 & 2607 & 868 & 258 \\
\hline 16 & 1747 & 1212 & 403 & 262 \\
\hline 22 & 2654 & 1155 & 384 & 261 \\
\hline 17 & 1751 & 1419 & 472 & 285 \\
\hline 3 & 2553 & 1263 & 420 & 286 \\
\hline 7 & 1504 & 1395 & 464 & 286 \\
\hline 18 & 2457 & 2361 & 786 & 256 \\
\hline
\end{tabular}




\begin{tabular}{|c|c|c|c|c|}
\hline 23 & 1206 & 1206 & 401 & 256 \\
\hline 13 & 2249 & 1872 & 623 & 256 \\
\hline 1 & 3983 & 3531 & 1176 & 256 \\
\hline 2 & 5 & NA & NA & 255 \\
\hline 25 & 2042 & 1164 & 387 & 269 \\
\hline 25 & 1717 & 1221 & 406 & 268 \\
\hline 23 & 2753 & 942 & 313 & 253 \\
\hline$x$ & 2155 & 1233 & 410 & 255 \\
\hline 5 & 1888 & 981 & 326 & 254 \\
\hline 21 & 3323 & 2553 & 850 & 257 \\
\hline 18 & 2894 & 2652 & 883 & 257 \\
\hline 11 & 5173 & 2673 & 890 & 257 \\
\hline 18 & 3390 & 1275 & 424 & 258 \\
\hline 17 & 1992 & 1605 & 534 & 267 \\
\hline 14 & 3504 & 1119 & 372 & 245 \\
\hline 11 & 3047 & 1032 & 343 & 245 \\
\hline 13 & 1988 & 1074 & 357 & 245 \\
\hline 7 & 5737 & 5643 & 1880 & 254 \\
\hline 2 & 10000 & 9846 & 3281 & 254 \\
\hline 1 & 8961 & 8961 & 2986 & 255 \\
\hline 20 & 4672 & 4212 & 1403 & 255 \\
\hline 7 & 1350 & 822 & 273 & 256 \\
\hline 17 & 1459 & 1104 & 367 & 261 \\
\hline 17 & 1548 & 1077 & 358 & 261 \\
\hline 2 & 1240 & 810 & 269 & 256 \\
\hline 10 & 1220 & 1017 & 338 & 281 \\
\hline 14 & 1639 & 1188 & 395 & 277 \\
\hline 7 & 1429 & 978 & 325 & 261 \\
\hline$x$ & 1903 & 1011 & 336 & 261 \\
\hline 19 & 3533 & 1230 & 409 & 261 \\
\hline 5 & 2133 & 1401 & 466 & 261 \\
\hline 10 & 1924 & 1314 & 437 & 264 \\
\hline 7 & 2383 & 1245 & 414 & 263 \\
\hline 7 & 4603 & 1341 & 446 & 262 \\
\hline 23 & 3834 & 3834 & 1277 & 254 \\
\hline 10 & 5466 & 3732 & 1243 & 256 \\
\hline 5 & 1525 & 1191 & 396 & 267 \\
\hline 11 & 2106 & 1296 & 431 & 264 \\
\hline 18 & 1956 & 1356 & 451 & 240 \\
\hline 28 & 1258 & 894 & 297 & 284 \\
\hline 5 & 1369 & 897 & 298 & 283 \\
\hline 19 & 1576 & 918 & 305 & 283 \\
\hline 18 & 1644 & 1086 & 361 & 285 \\
\hline 5 & 1369 & 912 & 303 & 290 \\
\hline 4 & 1619 & 981 & 326 & 288 \\
\hline 4 & 1100 & 879 & 292 & 283 \\
\hline 20 & 1376 & 1041 & 346 & 284 \\
\hline 9 & 2895 & 1503 & 500 & 316 \\
\hline 12 & 2484 & 1332 & 443 & 293 \\
\hline 11 & 1747 & 1119 & 372 & 297 \\
\hline
\end{tabular}




\begin{tabular}{|c|c|c|c|c|}
\hline 4 & 7268 & 4539 & 1512 & 294 \\
\hline 19 & 5981 & 4473 & 1490 & 294 \\
\hline 16 & 2916 & 2316 & 771 & 286 \\
\hline$X$ & 3105 & 1491 & 496 & 282 \\
\hline 5 & 3625 & 1572 & 523 & 282 \\
\hline 16 & 2847 & 1416 & 471 & 282 \\
\hline 4 & 1605 & 1486 & 494 & 285 \\
\hline 2 & 1305 & 1305 & 434 & 285 \\
\hline 8 & 2119 & 1041 & 346 & 285 \\
\hline 10 & 1889 & 1059 & 352 & 284 \\
\hline 6 & 2549 & 1710 & 569 & 284 \\
\hline 7 & 1776 & 1776 & 591 & 283 \\
\hline 11 & 1216 & 948 & 315 & 283 \\
\hline$x$ & 2949 & 2949 & 982 & 285 \\
\hline 2 & 1831 & 1458 & 485 & 317 \\
\hline 3 & 2131 & 1500 & 499 & 317 \\
\hline 21 & 1901 & 1473 & 490 & 317 \\
\hline 7 & 2081 & 1446 & 481 & 316 \\
\hline 1 & 5114 & 2292 & 763 & 321 \\
\hline 18 & 2567 & 2067 & 688 & 321 \\
\hline 5 & 1800 & 1800 & 599 & 314 \\
\hline 16 & 2180 & 1764 & 587 & 314 \\
\hline 5 & 1914 & 1914 & 637 & 297 \\
\hline 3 & 3645 & 3645 & 1214 & 331 \\
\hline 4 & 3606 & 3573 & 1190 & 329 \\
\hline 15 & 4874 & 3651 & 1216 & 329 \\
\hline 18 & 3360 & 1851 & 616 & 337 \\
\hline 23 & 4163 & 3027 & 1008 & 313 \\
\hline 18 & 2506 & 1488 & 495 & 285 \\
\hline 1 & 1861 & 1299 & 432 & 298 \\
\hline 25 & 2135 & 1089 & 362 & 289 \\
\hline 17 & 1272 & 1083 & 360 & 289 \\
\hline 10 & 3901 & 2169 & 722 & 297 \\
\hline 24 & 1755 & 1080 & 359 & 265 \\
\hline 19 & 3054 & 2346 & 781 & 293 \\
\hline 14 & 1959 & 1611 & 536 & 292 \\
\hline 28 & 3047 & 1284 & 427 & 296 \\
\hline 7 & 4602 & 1275 & 424 & 296 \\
\hline 6 & 2913 & 1281 & 426 & 296 \\
\hline 19 & 4073 & 1584 & 527 & 290 \\
\hline 23 & 4284 & 1083 & 360 & 285 \\
\hline 5 & 2290 & 1056 & 351 & 272 \\
\hline 23 & 1402 & 1101 & 366 & 284 \\
\hline 5 & 1727 & 1104 & 367 & 285 \\
\hline 23 & 4005 & 1887 & 628 & 281 \\
\hline 23 & 2257 & 1872 & 623 & 281 \\
\hline 21 & 1731 & 1302 & 433 & 282 \\
\hline$x$ & 2100 & 1698 & 565 & 486 \\
\hline 23 & 4291 & 1968 & 655 & 574 \\
\hline 4 & 5185 & 2133 & 710 & 606 \\
\hline
\end{tabular}




\begin{tabular}{|c|c|c|c|c|}
\hline 13 & 2000 & 1209 & 402 & 251 \\
\hline 19 & 2164 & 1035 & 344 & 251 \\
\hline 18 & 1071 & 915 & 304 & 251 \\
\hline 20 & 1053 & 1053 & 350 & 233 \\
\hline 11 & 3750 & 3237 & 1078 & 269 \\
\hline 10 & 4018 & 3189 & 1062 & 265 \\
\hline 13 & 1406 & 762 & 253 & 220 \\
\hline 19 & 2043 & 1518 & 505 & 285 \\
\hline 17 & 1957 & 1740 & 579 & 282 \\
\hline 3 & 3466 & 1731 & 576 & 514 \\
\hline 13 & 2328 & 1176 & 391 & 285 \\
\hline 18 & 1529 & 1053 & 350 & 286 \\
\hline 19 & 2999 & 2346 & 781 & 314 \\
\hline 26 & 3442 & 2223 & 740 & 274 \\
\hline 27 & 3490 & 2271 & 756 & 275 \\
\hline 16 & 3106 & 2157 & 718 & 318 \\
\hline 5 & 3348 & 2190 & 729 & 296 \\
\hline 19 & 2955 & 2931 & 976 & 262 \\
\hline 25 & 2937 & 2778 & 925 & 262 \\
\hline 14 & 1130 & 1023 & 340 & 272 \\
\hline 11 & 3195 & 2877 & 958 & 275 \\
\hline 6 & 3437 & 3375 & 1124 & 291 \\
\hline 6 & 4394 & 3807 & 1268 & 274 \\
\hline 2 & 1482 & 918 & 305 & 271 \\
\hline 8 & 1272 & NA & 424 & 255 \\
\hline 22 & 2505 & 2505 & 834 & 246 \\
\hline 1 & 1731 & 1731 & 576 & 259 \\
\hline 16 & 2142 & 1338 & 445 & 264 \\
\hline 12 & 2089 & 1488 & 495 & 254 \\
\hline 22 & 3480 & 2364 & 787 & 256 \\
\hline 12 & 1644 & 1644 & 547 & 256 \\
\hline 11 & 2550 & 942 & 313 & 261 \\
\hline 16 & 1680 & 912 & 303 & 262 \\
\hline 19 & 2147 & 2097 & 698 & 255 \\
\hline 10 & 5508 & 2934 & 977 & 257 \\
\hline 25 & 1077 & 1077 & 358 & 265 \\
\hline 18 & 1503 & 1503 & 500 & 264 \\
\hline 18 & 1095 & 1095 & 364 & 262 \\
\hline 2 & 2971 & 1767 & 588 & 352 \\
\hline 27 & 4155 & NA & 1384 & 313 \\
\hline 21 & 4647 & 4647 & 1548 & 337 \\
\hline 13 & 1540 & 1209 & 402 & 329 \\
\hline 2 & 1394 & 1290 & 429 & 333 \\
\hline 18 & 2025 & 2025 & 674 & 111 \\
\hline 19 & 4808 & 4482 & 1493 & 305 \\
\hline 11 & 2165 & 1608 & 535 & 264 \\
\hline 14 & 3298 & 1500 & 499 & 270 \\
\hline 3 & 2115 & 1260 & 419 & 282 \\
\hline 16 & 2779 & 2172 & 723 & 110 \\
\hline 11 & 1965 & 1965 & 654 & 266 \\
\hline
\end{tabular}




\begin{tabular}{|c|c|c|c|c|}
\hline 27 & 3345 & 1053 & 350 & 251 \\
\hline 4 & 2922 & 2922 & 973 & 237 \\
\hline 11 & 2142 & 1482 & 493 & 228 \\
\hline 16 & 5138 & 2787 & 928 & 264 \\
\hline 10 & 5179 & 4956 & 1651 & 412 \\
\hline 11 & 4607 & 3348 & 1115 & 484 \\
\hline 25 & 2860 & 1899 & 632 & 418 \\
\hline 11 & 2267 & 1602 & 533 & 256 \\
\hline 25 & 2187 & 1809 & 602 & 253 \\
\hline 20 & 2903 & 2058 & 685 & 253 \\
\hline 3 & 2371 & 1944 & 647 & 253 \\
\hline 17 & 3583 & 2682 & 893 & 254 \\
\hline 7 & 1941 & 1941 & 646 & 255 \\
\hline 29 & 2644 & 2424 & 807 & 231 \\
\hline 5 & 5581 & 2790 & 929 & 296 \\
\hline 16 & 2907 & 2259 & 752 & 221 \\
\hline 22 & 2927 & 1740 & 579 & 248 \\
\hline 6 & 2931 & 2931 & 976 & 256 \\
\hline 2 & 3897 & 2289 & 762 & 279 \\
\hline 19 & 3037 & 2313 & 770 & 280 \\
\hline 8 & 1972 & 975 & 324 & 288 \\
\hline 9 & 2971 & 2571 & 856 & 266 \\
\hline 2 & 4460 & 3948 & 1315 & 251 \\
\hline 17 & 3805 & 3168 & 1055 & 263 \\
\hline 19 & 3522 & 3114 & 1037 & 264 \\
\hline 21 & 2562 & 1419 & 472 & 257 \\
\hline 22 & 3788 & 3678 & 1225 & 277 \\
\hline 1 & 4994 & 4077 & 1358 & 284 \\
\hline 25 & 2579 & 1494 & 497 & 250 \\
\hline 15 & 2561 & 1269 & 422 & 271 \\
\hline 4 & 1701 & 1701 & 566 & 275 \\
\hline 5 & 8732 & NA & 2118 & 226 \\
\hline 8 & 6855 & 6681 & 2226 & 259 \\
\hline Un & 2460 & NA & 700 & 259 \\
\hline 19 & 4010 & 3660 & 1219 & 259 \\
\hline 3 & 3818 & 3195 & 1064 & 269 \\
\hline 8 & 3276 & 3144 & 1047 & 270 \\
\hline 19 & 4594 & 3333 & 1110 & 274 \\
\hline$X$ & 5956 & 3312 & 1103 & 280 \\
\hline 5 & 3772 & 3219 & 1072 & 273 \\
\hline 20 & 4881 & 4530 & 1509 & 268 \\
\hline 2 & 3025 & 1863 & 620 & 261 \\
\hline 19 & 2187 & 1851 & 616 & 261 \\
\hline 9 & 5630 & 4662 & 1553 & 259 \\
\hline 9 & 5283 & 4101 & 1366 & 261 \\
\hline 2 & 4109 & 3888 & 1295 & 254 \\
\hline$X$ & 5876 & 4473 & 1490 & 256 \\
\hline 2 & 3984 & 3984 & 1327 & 264 \\
\hline 22 & 1833 & 1584 & 527 & 275 \\
\hline 2 & 3229 & 1614 & 537 & 275 \\
\hline
\end{tabular}




\begin{tabular}{|c|c|c|c|c|}
\hline 29 & 2885 & 2466 & 821 & 258 \\
\hline 18 & 2653 & 2463 & 820 & 258 \\
\hline 10 & 4458 & 2541 & 846 & 258 \\
\hline 11 & 4184 & 2685 & 894 & 258 \\
\hline 11 & 4312 & 3999 & 1332 & 267 \\
\hline 1 & 6668 & 3918 & 1305 & 248 \\
\hline 19 & 4916 & 3933 & 1310 & 265 \\
\hline$x$ & 5847 & 4761 & 1586 & 289 \\
\hline 13 & 1911 & 1464 & 487 & 252 \\
\hline 14 & 3034 & 1830 & 609 & 252 \\
\hline 13 & 4908 & 4908 & 1635 & 267 \\
\hline 2 & 2985 & 2985 & 994 & 267 \\
\hline 29 & 3079 & 1635 & 544 & 252 \\
\hline 9 & 4292 & 1038 & 345 & 252 \\
\hline$x$ & 2336 & 1635 & 544 & 252 \\
\hline 18 & 2616 & 1782 & 593 & 252 \\
\hline 13 & 2511 & 2160 & 719 & 252 \\
\hline 10 & 3868 & 2046 & 681 & 252 \\
\hline 20 & 5256 & 2904 & 967 & 259 \\
\hline 26 & 3176 & 2967 & 988 & 259 \\
\hline 19 & 2294 & 1122 & 373 & 311 \\
\hline 2 & 2001 & 1299 & 432 & 312 \\
\hline 19 & 6255 & 3006 & 1001 & 254 \\
\hline 25 & 4145 & 3150 & 1049 & 254 \\
\hline 17 & 4101 & 2697 & 898 & 254 \\
\hline 12 & 1744 & 1302 & 433 & 251 \\
\hline$x$ & 1743 & 1365 & 454 & 251 \\
\hline 3 & 1618 & 1284 & 427 & 251 \\
\hline 10 & 2376 & 1182 & 393 & 294 \\
\hline 7 & 1633 & 1203 & 400 & 298 \\
\hline 19 & 2233 & 1044 & 347 & 262 \\
\hline 19 & 3848 & 1239 & 412 & 266 \\
\hline 10 & 1311 & 1311 & 436 & 253 \\
\hline 19 & 1527 & 1005 & 334 & 262 \\
\hline 7 & 3524 & 1407 & 468 & 261 \\
\hline 13 & 2553 & 1407 & 468 & 253 \\
\hline 19 & 4323 & 2862 & 953 & 267 \\
\hline 11 & 3952 & 3456 & 1151 & 252 \\
\hline 16 & 3539 & 3441 & 1146 & 252 \\
\hline 1 & 4276 & 3216 & 1071 & 260 \\
\hline 19 & 2611 & 1992 & 663 & 262 \\
\hline 11 & 4866 & 4866 & 1621 & 270 \\
\hline 18 & 4510 & 3012 & 1003 & 269 \\
\hline 11 & 3474 & 2928 & 975 & 266 \\
\hline 10 & 3746 & 2670 & 889 & 262 \\
\hline 23 & 4281 & 3450 & 1149 & 266 \\
\hline 21 & 2105 & 1353 & 450 & 246 \\
\hline 7 & 1895 & 1458 & 485 & 244 \\
\hline 23 & 3770 & 2748 & 915 & 295 \\
\hline 3 & 3105 & 2568 & 855 & 287 \\
\hline
\end{tabular}




\begin{tabular}{|c|c|c|c|c|}
\hline 22 & 3633 & 3633 & 1210 & 258 \\
\hline 19 & 3786 & 3786 & 1261 & 258 \\
\hline 5 & 5448 & 4011 & 1336 & 257 \\
\hline 2 & 3459 & 2190 & 1152 & 258 \\
\hline 4 & 3207 & 2952 & 983 & 260 \\
\hline 3 & 3027 & 3027 & 1008 & 255 \\
\hline 2 & 3264 & 2928 & 975 & 257 \\
\hline 1 & 3050 & 2955 & 984 & 257 \\
\hline 2 & 3313 & 2973 & 990 & 257 \\
\hline 6 & 1712 & NA & 569 & 257 \\
\hline 1 & 3182 & NA & 1059 & 300 \\
\hline 9 & 3321 & 2997 & 998 & 257 \\
\hline 2 & 3156 & 3156 & 1051 & 258 \\
\hline 1 & 3830 & 2889 & 962 & 260 \\
\hline 3 & 504 & 504 & 167 & 85 \\
\hline 2 & 3886 & 2898 & 965 & 260 \\
\hline 1 & 3784 & 2994 & 997 & 260 \\
\hline 25 & 3975 & 3021 & 1006 & 285 \\
\hline 4 & 3175 & 2979 & 992 & 247 \\
\hline 14 & 4541 & 3186 & 1061 & 264 \\
\hline 8 & 4131 & 3030 & 1009 & 260 \\
\hline 7 & 2636 & 2469 & 822 & 254 \\
\hline$X$ & 516 & NA & 172 & 138 \\
\hline 21 & 2739 & 2469 & 822 & 256 \\
\hline 27 & 3400 & 2463 & 820 & 277 \\
\hline 26 & 3944 & 2469 & 822 & 277 \\
\hline 6 & 2562 & 2409 & 802 & 277 \\
\hline 7 & 2974 & 2403 & 800 & 277 \\
\hline 21 & 4483 & 3615 & 1204 & 270 \\
\hline 7 & 4770 & 3876 & 1291 & 268 \\
\hline 3 & 3906 & 3906 & 1301 & 270 \\
\hline 3 & 4331 & 3477 & 1158 & 278 \\
\hline 8 & 4695 & 3399 & 1132 & 278 \\
\hline 7 & 3775 & 3318 & 1105 & 283 \\
\hline 7 & 5781 & 3558 & 1185 & 266 \\
\hline 19 & 4706 & 4104 & 1367 & 274 \\
\hline 25 & 4751 & NA & 1422 & 275 \\
\hline 18 & 4631 & NA & 1431 & 320 \\
\hline 4 & 4823 & 4155 & 1384 & 261 \\
\hline 22 & 4489 & 4206 & 1401 & 253 \\
\hline 8 & 3259 & 2610 & 869 & 281 \\
\hline 12 & 3416 & 2925 & 974 & 334 \\
\hline 7 & 3825 & 2895 & 964 & 330 \\
\hline 6 & 5078 & 2967 & 988 & 336 \\
\hline 6 & 3270 & 3270 & 1089 & 358 \\
\hline 7 & 4452 & 3312 & 1103 & 359 \\
\hline 28 & 3541 & 3342 & 1113 & 284 \\
\hline 3 & 3299 & 2727 & 908 & 307 \\
\hline 8 & 4006 & 2823 & 940 & 307 \\
\hline 1 & 2593 & 1617 & 538 & 267 \\
\hline
\end{tabular}




\begin{tabular}{|c|c|c|c|c|}
\hline 8 & 660 & 660 & 219 & 218 \\
\hline 8 & 2114 & 1506 & 501 & 251 \\
\hline 13 & 1353 & 1353 & 450 & 253 \\
\hline 2 & 2195 & 1584 & 527 & 250 \\
\hline 9 & 3436 & 1545 & 514 & 258 \\
\hline 9 & 2409 & 1614 & 537 & 250 \\
\hline 13 & 2046 & 1512 & 503 & 251 \\
\hline 2 & 2080 & 1530 & 509 & 251 \\
\hline 14 & 2750 & 1539 & 512 & 252 \\
\hline 13 & 1697 & 1629 & 542 & 249 \\
\hline 13 & 1485 & 1485 & 494 & 251 \\
\hline 24 & 4997 & 1626 & 541 & 250 \\
\hline 8 & 2532 & 1818 & 605 & 249 \\
\hline 11 & 3947 & 1854 & 617 & 249 \\
\hline$x$ & 1965 & 1965 & 654 & 252 \\
\hline$X$ & 2577 & 1980 & 659 & 252 \\
\hline 7 & 4393 & 1863 & 620 & 250 \\
\hline 6 & 2538 & 1956 & 651 & 250 \\
\hline 6 & 2312 & 1584 & 527 & 252 \\
\hline 3 & 3631 & 3411 & 1136 & 269 \\
\hline 8 & 4625 & 3378 & 1125 & 269 \\
\hline 5 & 1592 & 1269 & 422 & 269 \\
\hline 3 & 2603 & 2388 & 795 & 275 \\
\hline 8 & 3538 & 2514 & 837 & 273 \\
\hline 21 & 3380 & 2478 & 825 & 272 \\
\hline 12 & 4080 & 4080 & 1359 & 328 \\
\hline 7 & 4383 & 4068 & 1355 & 325 \\
\hline 6 & 5998 & 4071 & 1356 & 327 \\
\hline$x$ & 2215 & 2157 & 718 & 310 \\
\hline 22 & 2823 & 1866 & 621 & 268 \\
\hline 5 & 2177 & 1743 & 580 & 279 \\
\hline 5 & 2590 & 1386 & 461 & 269 \\
\hline 25 & 3086 & 1944 & 647 & 266 \\
\hline 17 & 2274 & 1917 & 638 & 271 \\
\hline 8 & 2192 & 1893 & 630 & 258 \\
\hline 3 & 1569 & 1569 & 522 & 260 \\
\hline 21 & 6561 & 6048 & 2015 & 283 \\
\hline 5 & 7871 & 7608 & 2535 & 246 \\
\hline 3 & 3020 & 2508 & 835 & 261 \\
\hline 5 & 3332 & 2685 & 894 & 239 \\
\hline 1 & 3425 & 2901 & 966 & 240 \\
\hline 10 & 3312 & 3312 & 1103 & 260 \\
\hline 18 & 1077 & 1077 & 358 & 193 \\
\hline 29 & 3595 & 2595 & 864 & 260 \\
\hline 28 & 3147 & 3147 & 1048 & 275 \\
\hline 2 & 2778 & 2403 & 800 & 244 \\
\hline 9 & 3388 & 1740 & 579 & 248 \\
\hline$X$ & 2355 & 1821 & 606 & 258 \\
\hline 4 & 2492 & 2298 & 765 & 260 \\
\hline 19 & 2913 & 2334 & 777 & 264 \\
\hline
\end{tabular}




\begin{tabular}{|c|c|c|c|c|}
\hline 17 & 1836 & 1836 & 611 & 262 \\
\hline 22 & 3209 & 2007 & 668 & 260 \\
\hline 1 & 3794 & 2358 & 785 & 264 \\
\hline 23 & 2403 & 1998 & 665 & 269 \\
\hline 14 & 1688 & 1623 & 540 & 267 \\
\hline 10 & 1802 & 1512 & 503 & 261 \\
\hline 15 & 1884 & NA & 628 & 215 \\
\hline 5 & 2494 & 1512 & 503 & 288 \\
\hline 2 & 2808 & 1530 & 509 & 288 \\
\hline 5 & 2578 & 1518 & 505 & 288 \\
\hline 2 & 1662 & 1479 & 492 & 288 \\
\hline 28 & 4059 & 1599 & 532 & 288 \\
\hline 6 & 3498 & 1509 & 502 & 288 \\
\hline 8 & 2060 & 1500 & 499 & 288 \\
\hline 2 & 4428 & 1542 & 513 & 281 \\
\hline 22 & 1833 & 1539 & 512 & 307 \\
\hline 2 & 3823 & 2745 & 914 & 312 \\
\hline 5 & 1716 & 1716 & 571 & 295 \\
\hline 22 & 4280 & 1704 & 567 & 257 \\
\hline 15 & 1701 & 1359 & 452 & 263 \\
\hline 18 & 2323 & 1407 & 468 & 177 \\
\hline 14 & 759 & 759 & 252 & \\
\hline
\end{tabular}




\begin{tabular}{|c|c|c|c|}
\hline VAIK & HRD & DFG & Catalytic activity \\
\hline YAMK & YRD & DFG & Active \\
\hline YAMK & YRD & DFG & Active \\
\hline YAMK & YRD & DFG & Active \\
\hline YAMK & HRD & DFG & Active \\
\hline FAMK & HRD & DFG & Active \\
\hline FAMK & HRD & DFG & Active \\
\hline YAMK & HRD & DFG & Active \\
\hline YAMK & HRD & DFD & Active \\
\hline \multirow[t]{2}{*}{ YAMK } & HRD & DFD & Active \\
\hline & & & partial \\
\hline YAMK & YRD & DLG & Active \\
\hline YAMK & YRD & DLG & Active \\
\hline YACK & YRD & DLG & Active \\
\hline YACK & YRD & DLG & Active \\
\hline YACK & YRD & DLG & Active \\
\hline YACK & YRD & DLG & Active \\
\hline YACK & YRD & DLG & Active \\
\hline FAMK & $\mathrm{HRD}$ & DFG & Active \\
\hline FAMK & HRD & DFG & Active \\
\hline FAIK & HRD & DFG & Active \\
\hline FAMK & HRD & DFG & Active \\
\hline YAVK & HRD & DFG & Active \\
\hline--- & HRD & DFG & Inactive \\
\hline YATK & HRD & DFG & Active \\
\hline YAMK & HRD & DFG & Active \\
\hline YAMK & $\mathrm{HRD}$ & DFG & Active \\
\hline YAMK & $\mathrm{HRD}$ & DFG & Active \\
\hline YAMK & YRD & DFG & Active \\
\hline YAMK & YRD & DFG & Active \\
\hline FALK & YRD & DFG & Active \\
\hline YAIK & HRD & DFG & Active \\
\hline YAMK & YRD & DYG & Active \\
\hline YAMK & YRD & DYG & Active \\
\hline YAIK & YRD & DFG & Active \\
\hline YAVK & YRD & DFG & Active \\
\hline YAIK & YRD & DFG & Active \\
\hline FAIK & YRD & DFG & Active \\
\hline YAVK & YRD & DFG & Active \\
\hline YAVK & YRD & DFG & Active \\
\hline FAIK & YRD & DFG & Active \\
\hline FAMK & YRD & DFG & Active \\
\hline FAMK & YRD & DFG & Active \\
\hline FAIK & YRD & DFG & Active \\
\hline FAIK & YRD & DFG & Active \\
\hline YAIK & YRD & DFG & Active \\
\hline YAMK & YRD & DFG & Active \\
\hline YAMK & YRD & DFG & Active \\
\hline FAMK & YRD & DFG & Active \\
\hline YAMK & YRD & DFG & Active \\
\hline
\end{tabular}




\begin{tabular}{|c|c|c|c|}
\hline YAMK & YRD & DFG & Active \\
\hline YAMK & YRD & DFG & Active \\
\hline YAMK & YRD & DFG & Active \\
\hline YAMK & YRD & DFG & Active \\
\hline LKTE & CRD & YFS & Inactive \\
\hline LHLE & CRD & YFG & Inactive \\
\hline FAVK & HRD & DFG & Active \\
\hline YAVK & YRD & DFG & Active \\
\hline YAVK & YRD & DFG & Active \\
\hline YAVK & YRD & DFG & Active \\
\hline YMNK & HRD & DFN & Active \\
\hline YAMK & HRD & DFN & Active \\
\hline \multirow[t]{29}{*}{ YAMK } & HRD & DFN & Active \\
\hline & & & Atypical \\
\hline & & & Atypical \\
\hline & & & Atypical \\
\hline & & & Atypical \\
\hline & & & Atypical \\
\hline & & & Atypical \\
\hline & & & Atypical \\
\hline & & & Atypical \\
\hline & & & Atypical \\
\hline & & & Atypical \\
\hline & & & Atypical \\
\hline & & & Atypical \\
\hline & & & Atypical \\
\hline & & & Atypical \\
\hline & & & Atypical \\
\hline & & & Atypical \\
\hline & & & Atypical \\
\hline & & & Atypical \\
\hline & & & Atypical \\
\hline & & & Atypical \\
\hline & & & Atypical \\
\hline & & & Atypical \\
\hline & & & Atypical \\
\hline & & & Atypical \\
\hline & & & Atypical \\
\hline & & & Atypical \\
\hline & & & Atypical \\
\hline & & & Atypical \\
\hline \multirow[t]{9}{*}{ FLVK } & IGDRH & DFG & Active \\
\hline & & & Atypical \\
\hline & & & Atypical \\
\hline & & & Atypical \\
\hline & & & Atypical \\
\hline & & & Atypical \\
\hline & & & Atypical \\
\hline & & & Atypical \\
\hline & & & Atypical \\
\hline
\end{tabular}




\begin{tabular}{|c|c|c|c|}
\hline & & & Atypical \\
\hline & & & Atypical \\
\hline VAIK & HRD & DFG & Active \\
\hline VALK & HRD & DFG & Active \\
\hline FAVK & HRD & DFG & Active \\
\hline FALK & HRD & DFG & Active \\
\hline YAAK & HRD & DFG & Active \\
\hline YAAK & HRD & DFG & Active \\
\hline YAAK & HRD & DFG & Active \\
\hline YAAK & HRD & DFG & Active \\
\hline YALK & HRD & DRG & Active \\
\hline VAVK & HRD & DFG & Active \\
\hline VAVK & HRD & DFG & Active \\
\hline VAIK & HRD & DFG & Active \\
\hline VAVK & HRD & DFG & Active \\
\hline VAVK & HRD & DFG & Active \\
\hline VAIK & HRD & DFG & Active \\
\hline RAVK & HKD & DLG & Active \\
\hline VAVK & HRD & DFG & Active \\
\hline VAVK & HRD & DFG & Active \\
\hline VAIK & HRD & DFG & Active \\
\hline VAIK & HRD & DFG & Active \\
\hline VAIK & HRD & DFG & Active \\
\hline VAIK & HRD & DFG & Active \\
\hline VAIK & HRD & DFG & Active \\
\hline VAIK & HRD & DFG & Active \\
\hline VAIK & HRD & DFG & Active \\
\hline VVVK & HRD & DFG & Active \\
\hline VAIK & HRD & DFG & Active \\
\hline VAIK & HRD & DFG & Active \\
\hline VAIK & HRD & DFG & Active \\
\hline VAVK & HRD & DFG & Active \\
\hline YVIK & FYD & DGR & Active \\
\hline WAIK & HRD & DFG & Active \\
\hline HTCK & HRN & DFH & Inactive \\
\hline FAVK & HRD & GFG & Inactive \\
\hline YAAK & HFD & DFG & Active \\
\hline YAAK & HFD & DFG & Active \\
\hline YAAK & HFD & DFG & Active \\
\hline FAAK & HLD & DFG & Active \\
\hline YAAK & HLD & DFG & Active \\
\hline YALK & HRD & DFG & Active \\
\hline FALK & HRD & DFG & Active \\
\hline YAMK & HRD & DFG & Active \\
\hline FALK & HRD & DFG & Active \\
\hline CALK & HRD & DFG & Active \\
\hline FALK & HRD & DFG & Active \\
\hline YAVK & HRD & DLG & Active \\
\hline YAVK & HRD & DLG & Active \\
\hline LAAK & HLD & DFG & Active \\
\hline
\end{tabular}




\begin{tabular}{|c|c|c|c|}
\hline LAAK & HLD & DFG & Active \\
\hline LAAK & HLD & DFG & Active \\
\hline WAGK & HLD & DFG & Active \\
\hline YMAK & HFD & EFG & Inactive \\
\hline YAVK & HRD & DFG & Active \\
\hline FAVK & HRD & DFG & Active \\
\hline VAIK & HRD & DFG & Active \\
\hline VAIK & HRD & DFG & Active \\
\hline VAVK & HRD & DFG & Active \\
\hline VAIK & $\mathrm{HCD}$ & DFG & Active \\
\hline VAVK & $\mathrm{HCD}$ & DFG & Active \\
\hline VAIK & $\mathrm{HCD}$ & DFG & Active \\
\hline YAIK & HRD & DFG & Active \\
\hline VAIK & HRD & DFG & Active \\
\hline LRCK & LGD & SLE & Inactive \\
\hline LVCK & LRD & SLE & Inactive \\
\hline YICK & LRD & NLE & Inactive \\
\hline CAAK & HLD & DFG & Active \\
\hline FAAK & HLD & DFG & Active \\
\hline VAVK & HLD & DLE & Active \\
\hline VATK & HLD & DFG & Active \\
\hline VAIK & HRD & DFG & Active \\
\hline VAVK & HRD & DFG & Active \\
\hline VAVK & HRD & DFG & Active \\
\hline VAIK & HRD & DFG & Active \\
\hline VAVK & GRD & DFG & Active \\
\hline VAIK & HRD & DFG & Active \\
\hline VAVK & HRD & DFG & Active \\
\hline VAVK & HRD & DFG & Active \\
\hline VAIK & HRD & DFG & Active \\
\hline VAIK & HRD & DFG & Active \\
\hline VAIK & YRD & DFG & Active \\
\hline VAIK & YRD & DFG & Active \\
\hline VAIK & YRD & DFG & Active \\
\hline VALK & HRD & DFG & Active \\
\hline VALK & HRD & DFG & Active \\
\hline CVVK & HGD & DYG & Active \\
\hline HVIK & HGD & DYG & Active \\
\hline HQDK & $\mathrm{HGN}$ & GYG & Inactive \\
\hline VAMK & HRD & DFG & Active \\
\hline VALK & HRD & DFG & Active \\
\hline VALK & HRD & DFG & Active \\
\hline VALK & HRD & DFG & Active \\
\hline VALK & HRD & DFG & Active \\
\hline VALK & HRD & DFG & Active \\
\hline VALK & HRD & DFG & Active \\
\hline VAIK & HRD & DFG & Active \\
\hline YALK & HRD & DMG & Active \\
\hline YALK & HRD & DMG & Active \\
\hline VALK & HRD & DFG & Active \\
\hline
\end{tabular}




\begin{tabular}{|c|c|c|c|}
\hline VALK & HRD & DFG & Active \\
\hline VALK & HRD & DFG & Active \\
\hline VALK & HRD & DFG & Active \\
\hline VALK & HRD & DFG & Active \\
\hline VALK & HRD & DFG & Active \\
\hline VALK & HRD & DFG & Active \\
\hline VALK & HRD & DFG & Active \\
\hline VALK & HRD & DFG & Active \\
\hline VALK & HRD & DFG & Active \\
\hline VAIK & HRD & DFG & Active \\
\hline VAIK & HRD & DFG & Active \\
\hline VAIK & HRD & DFG & Active \\
\hline VAIK & HRD & DFG & Active \\
\hline VELK & HRD & DFG & Active \\
\hline VAVK & HTD & DFG & Active \\
\hline VALK & HTD & DWD & Active \\
\hline VALK & HTD & DFG & Active \\
\hline VAVK & HTD & DFG & Active \\
\hline VAIK & $\mathrm{HCD}$ & DFG & Active \\
\hline VAIK & $\mathrm{HCD}$ & DFG & Active \\
\hline VALK & $\mathrm{HCD}$ & DFG & Active \\
\hline VALK & $\mathrm{HCD}$ & DFG & Active \\
\hline VALK & $\mathrm{HCD}$ & DFG & Active \\
\hline VAIK & HAD & DFG & Active \\
\hline VAIK & HAD & DFG & Active \\
\hline VAIK & HAD & DFG & Active \\
\hline VAIK & HAD & DFG & Active \\
\hline VAVK & HAD & DFG & Active \\
\hline VAIK & HRD & DFG & Active \\
\hline VAIK & HRD & DFG & Active \\
\hline VAIK & HRD & DFG & Active \\
\hline VAIK & HRD & DFG & Active \\
\hline VAIK & HRD & DFG & Active \\
\hline VAVK & HRD & DFG & Active \\
\hline VAIK & HRD & DFG & Active \\
\hline VAIK & HRD & DFG & Active \\
\hline VAIK & HRD & DFG & Active \\
\hline VAVK & HRD & DFG & Active \\
\hline VAIK & HRD & DFG & Active \\
\hline VALK & HRD & DFG & Active \\
\hline VAVK & HRD & DFG & Active \\
\hline VAVK & HRD & DFG & Active \\
\hline VAIK & HRD & DFG & Active \\
\hline VAIK & HRD & DFG & Active \\
\hline IAIK & HRD & DFG & Active \\
\hline VAIK & HRD & DFG & Active \\
\hline YACK & HRD & DFG & Active \\
\hline VALK & HTD & DLG & Active \\
\hline VAMK & HTD & DLG & Active \\
\hline VAMK & HTD & DLG & Active \\
\hline
\end{tabular}




\begin{tabular}{|c|c|c|c|}
\hline LALK & HRD & DFG & Active \\
\hline VALK & HRD & DFG & Active \\
\hline VALK & HRD & DFG & Active \\
\hline VTLK & RRD & LIS & Inactive \\
\hline FALK & HGD & DLG & Active \\
\hline TIIK & HGD & DFS & Active \\
\hline AVVK & HGD & DFG & Active \\
\hline YAMK & $\mathrm{HRD}$ & DFG & Active \\
\hline YAMK & HRD & DFG & Active \\
\hline IALK & HRD & DFG & Active \\
\hline VVVK & HRD & DWG & Active \\
\hline VVVK & HRD & DWG & Active \\
\hline VALK & HRD & TLN & Inactive \\
\hline IAIK & HRD & DLG & Active \\
\hline IAIK & HRD & DLG & Active \\
\hline VAVK & HRD & DFG & Active \\
\hline FAIK & HRD & DFG & Active \\
\hline VAVK & HRD & DFG & Active \\
\hline VAVK & HRD & DFG & Active \\
\hline VAVK & HLD & DFG & Active \\
\hline CALK & HRD & DFG & Active \\
\hline YALK & HRD & DFG & Active \\
\hline YALK & HRD & DFG & Active \\
\hline YALK & HRD & DLG & Active \\
\hline YVIK & HRD & DFG & Active \\
\hline LAMK & HRD & DFG & Active \\
\hline KVLK & HRD & DFG & Active \\
\hline LVWK & HRD & DFG & Active \\
\hline FAMK & HRD & DFG & Active \\
\hline YVIK & HRD & DLG & Active \\
\hline CVIK & HRD & DFG & Active \\
\hline VALK & HRD & DLG & Active \\
\hline VALK & HRD & DLG & Active \\
\hline VIIK & HRD & DFG & Active \\
\hline VVWK & HRD & DYG & Active \\
\hline MALK & HRD & DFG & Active \\
\hline LALK & YRD & DFG & Active \\
\hline VALK & HAD & DLG & Active \\
\hline LAIK & HRD & DFG & Active \\
\hline YAVK & HRD & NFL & Inactive \\
\hline YAVK & $\mathrm{HCD}$ & NFS & Inactive \\
\hline VAVK & HRD & DFG & Active \\
\hline VAVK & HRD & DFG & Active \\
\hline VKAK & & & partial \\
\hline VTVK & HRS & NLE & Inactive \\
\hline VVWN & HGN & VAP & Inactive \\
\hline VVWN & HGN & VWH & Inactive \\
\hline GALK & HAD & DFG & Active \\
\hline VAVK & HQD & DFD & Active \\
\hline TKVK & HRN & DLS & Inactive \\
\hline
\end{tabular}




\begin{tabular}{|c|c|c|c|}
\hline VALS & TRV & NFS & Inactive \\
\hline ILLK & HGS & DFD & Inactive \\
\hline VALK & LLD & DLD & Active \\
\hline CALK & HRD & DLG & Active \\
\hline YAVK & HRD & DFG & Active \\
\hline YAIK & HRD & DFG & Active \\
\hline YAIK & HRD & DFG & Active \\
\hline YVIK & HRD & DFG & Active \\
\hline FAGK & HRD & DFG & Active \\
\hline YAAK & HRD & DFG & Active \\
\hline YAVK & HRD & DFG & Active \\
\hline VAIK & HRD & DFG & Active \\
\hline FALK & HRD & DLG & Active \\
\hline VSIF & HNN & GLD & Inactive \\
\hline VAVF & HGN & GFD & Inactive \\
\hline ASVF & ARD & GME & Inactive \\
\hline LVLS & YGH & DLE & Inactive \\
\hline LTPN & HRA & KFG & Inactive \\
\hline AAVK & HYD & DFG & Active \\
\hline VAVK & HYD & DFG & Active \\
\hline WAVK & HGD & DVG & Active \\
\hline HAIK & HSD & DFG & Active \\
\hline VALK & HRD & DFG & Active \\
\hline VAVK & HRD & DFG & Active \\
\hline VAIK & HRD & DFG & Active \\
\hline VAIK & HLD & DFG & Active \\
\hline VAIL & FCD & NFC & Inactive \\
\hline VVVK & HGD & DFA & Active \\
\hline YAVK & HLD & DFG & Active \\
\hline YAIK & HMD & DLG & Active \\
\hline YAIK & HLD & DLG & Active \\
\hline VAWC & HRD & DLG & Active \\
\hline VAWC & HRD & DLG & Active \\
\hline VAWC & HRD & DLG & Active \\
\hline VAWC & HRD & DLG & Active \\
\hline VAVK & HGN & DYG & Inactive \\
\hline VAIK & HGS & DYG & Inactive \\
\hline VWLK & HGR & DHG & Inactive \\
\hline VWLK & HGR & DYG & Inactive \\
\hline VILK & HGR & DFG & Inactive \\
\hline MAVK & HRD & DFG & Active \\
\hline LAVK & HRD & DFG & Active \\
\hline LASK & HRD & DFG & Active \\
\hline MAMK & HRD & DFG & Active \\
\hline IAIK & HRD & DFG & Active \\
\hline IAIK & HRD & DFG & Active \\
\hline IAIK & HRD & DFG & Active \\
\hline IAVK & HRD & DFG & Active \\
\hline VAIK & HRD & DFG & Active \\
\hline VAIK & HRD & DFG & Active \\
\hline
\end{tabular}




\begin{tabular}{|c|c|c|c|}
\hline AAVK & HRD & DFG & Active \\
\hline VALK & HRD & DFG & Active \\
\hline AAVK & HRD & DFG & Active \\
\hline AAIK & HRD & DFG & Active \\
\hline AAIK & HRD & DFG & Active \\
\hline AAIK & HRD & DFG & Active \\
\hline AAIK & HRD & DFG & Active \\
\hline TAVK & HRD & DFG & Active \\
\hline VAIK & HRD & DFG & Active \\
\hline VAIK & HRD & DFG & Active \\
\hline AAVK & HRD & DFG & Active \\
\hline AAVK & HRD & DFG & Active \\
\hline VAIK & HRD & DFG & Active \\
\hline VAIK & HRD & DFG & Active \\
\hline VAIK & HRD & DFG & Active \\
\hline VAVK & HRD & DFG & Active \\
\hline VAVK & HRD & DFG & Active \\
\hline VAVK & HRD & DFG & Active \\
\hline AAAK & HRD & DFG & Active \\
\hline AAAK & HRD & DFG & Active \\
\hline VTVR & HRS & GLR & Inactive \\
\hline VTIK & HRS & GLS & Inactive \\
\hline VAIK & HRD & DFG & Active \\
\hline VAIK & HRD & DFG & Active \\
\hline VAIK & HRD & DFG & Active \\
\hline VAIK & HRD & DFG & Active \\
\hline VAIK & HRD & DFG & Active \\
\hline VAIK & HRD & DFG & Active \\
\hline MARK & HRD & DFG & Active \\
\hline MARK & HRD & DFG & Active \\
\hline MAVK & HRD & DFG & Active \\
\hline MAVK & HRD & DFG & Active \\
\hline LAVK & HRD & DFG & Active \\
\hline MAVK & HRD & DFG & Active \\
\hline IAVK & HRD & DFG & Active \\
\hline MACK & HHD & DFG & Active \\
\hline CAVK & HGD & DFG & Active \\
\hline VAVK & HRD & DFG & Active \\
\hline VAVK & HRD & DFG & Active \\
\hline VAVK & HRD & DFG & Active \\
\hline VAVK & HRD & DFG & Active \\
\hline VAVK & HRD & DFG & Active \\
\hline VAVK & HRD & DFG & Active \\
\hline VAVK & HRD & DFG & Active \\
\hline VAVK & HRD & DFG & Active \\
\hline VLVK & HKD & ALG & Inactive \\
\hline VAVK & HRD & DFG & Active \\
\hline VAVK & HRD & DFG & Active \\
\hline VAVK & HRD & DFG & Active \\
\hline VAVK & HRD & DFG & Active \\
\hline
\end{tabular}




\begin{tabular}{|c|c|c|c|}
\hline VAIK & HRD & DFG & Active \\
\hline VAIK & HRD & DFG & Active \\
\hline VCIK & HRN & DFG & Inactive \\
\hline VAIK & HRD & DFG & Active \\
\hline VAIK & HRD & DFG & Active \\
\hline VAVH & HRG & GFG & Inactive \\
\hline VAIK & HRD & DFG & Active \\
\hline VAIK & HRD & DFG & Active \\
\hline VAIK & HRD & DFG & Active \\
\hline VAIK & HRD & DFG & Active \\
\hline VAIK & HRD & DFG & Active \\
\hline VAIK & HRD & DFG & Active \\
\hline VAIK & HRD & DFG & Active \\
\hline \multirow[t]{2}{*}{ VAIK } & HRD & DFG & Active \\
\hline & & & partial \\
\hline VAIK & HRD & DFG & Active \\
\hline VAIK & HRD & DFG & Active \\
\hline VAIK & HRD & DFG & Active \\
\hline VAIQ & HRS & RLG & Inactive \\
\hline VAIK & HRD & DFG & Active \\
\hline VAVK & HRD & DFG & Active \\
\hline \multirow[t]{2}{*}{ VAVK } & HRD & DFG & Active \\
\hline & HRD & DFG & partial \\
\hline VAVK & HRD & DFG & Active \\
\hline VAVK & HRD & DFG & Active \\
\hline VAVK & HRD & DFG & Active \\
\hline VAVK & HRD & DFG & Active \\
\hline VAVK & HRD & DFG & Active \\
\hline VAIK & HRD & DFG & Active \\
\hline VAVK & HRD & DFG & Active \\
\hline VALK & HRD & DFG & Active \\
\hline VAVK & HRD & DFG & Active \\
\hline VAVK & HRD & DFG & Active \\
\hline VAVK & HRD & DFG & Active \\
\hline VAVK & HRD & DFG & Active \\
\hline VVVK & HSD & DYG & Active \\
\hline VIVK & HSD & DYG & Active \\
\hline VVVK & HSD & DYG & Active \\
\hline CAVK & HRD & DFG & Active \\
\hline CAIK & HRD & DFG & Active \\
\hline VAVK & HRD & DFG & Active \\
\hline VAVK & HRD & DFG & Active \\
\hline VAVK & HRD & DFG & Active \\
\hline VAVK & HRD & DFG & Active \\
\hline VAVK & HRD & DFG & Active \\
\hline VAVK & HRD & DFG & Active \\
\hline VAVK & HRD & DFG & Active \\
\hline VAIK & HKD & DLG & Active \\
\hline VAIK & HKD & DLG & Active \\
\hline AFVK & HKD & DNA & Active \\
\hline
\end{tabular}




\begin{tabular}{|c|c|c|c|}
\hline AFVK & HQD & DNA & Active \\
\hline VAIK & HRD & DFG & Active \\
\hline VAIK & HRD & DFG & Active \\
\hline VAVK & HRD & DFG & Active \\
\hline VAVK & HRD & DFG & Active \\
\hline VAIK & HRD & DFG & Active \\
\hline VAVK & HRD & DFG & Active \\
\hline VAVK & HRD & DFG & Active \\
\hline VAVK & HRD & DFG & Active \\
\hline VAIK & HRD & DFG & Active \\
\hline VAVK & HRD & DFG & Active \\
\hline VAIK & HRD & DFG & Active \\
\hline VAVK & HRD & DFG & Active \\
\hline VAIK & HRD & DFG & Active \\
\hline VAVK & HRD & DFG & Active \\
\hline VAIK & HRD & DFG & Active \\
\hline VAIK & HRD & DFG & Active \\
\hline VAIK & HRD & DFG & Active \\
\hline VAIK & HRD & DFG & Active \\
\hline AAIK & HRD & DFG & Active \\
\hline AAIK & HRD & DFG & Active \\
\hline VTLK & HGD & GLG & Inactive \\
\hline VAVK & HRD & DFG & Active \\
\hline VAVK & HRD & DFG & Active \\
\hline VAVK & HRD & DFG & Active \\
\hline VAVK & HRD & DFG & Active \\
\hline VAVK & HRD & DFG & Active \\
\hline VAVK & HRD & DFG & Active \\
\hline YAVK & HGD & DFG & Active \\
\hline FVFK & HSN & HSM & Inactive \\
\hline YAIK & CGS & DFA & Inactive \\
\hline VAVK & HRD & DFG & Active \\
\hline QAIK & HRD & DFG & Active \\
\hline QAIK & HRD & DFG & Active \\
\hline MVLK & HRD & DFG & Active \\
\hline NVHK & HRD & DFG & Active \\
\hline VAVK & FCD & DYG & Active \\
\hline VAVK & YRD & DYG & Active \\
\hline VAIK & HRD & DFG & Active \\
\hline VAVK & HRD & DFG & Active \\
\hline VAIK & HRD & DFG & Active \\
\hline VAVK & HRD & DFG & Active \\
\hline TVLK & EID & DFG & Active \\
\hline VAVK & HRD & DFG & Active \\
\hline VAVK & HRD & DFG & Active \\
\hline VAVK & HRD & DFG & Active \\
\hline VAIK & HRD & DFG & Active \\
\hline VAVK & HRD & DFG & Active \\
\hline VAVK & HRD & DFG & Active \\
\hline VAIR & HKD & DFG & Inactive \\
\hline
\end{tabular}




\begin{tabular}{|c|c|c|c|}
\hline VAIR & HKD & DFG & Inactive \\
\hline VAVK & HRD & DFG & Active \\
\hline LAIK & HLD & DFG & Active \\
\hline VILK & HRD & DLG & Active \\
\hline VAVK & HHD & DFG & Active \\
\hline VAVK & HRD & DFG & Active \\
\hline EAAK & HLD & DFG & Active \\
\hline VAVK & HRD & DLG & Active \\
\hline VAVK & HRD & DLG & Active \\
\hline VAVK & HRD & DLG & Active \\
\hline VAVK & HRD & DLG & Active \\
\hline VAVK & HRD & DLG & Active \\
\hline VAVK & HRD & DLG & Active \\
\hline VAVK & HRD & DLG & Active \\
\hline VAVK & HRD & DFG & Active \\
\hline VAVK & HRD & DFG & Active \\
\hline VAVK & HRD & DFG & Active \\
\hline VAIK & HRD & DLG & Active \\
\hline VAVK & HRD & DFG & Active \\
\hline IVVK & RHA & --- & Inactive \\
\hline VAIK & HRN & ELS & Inactive \\
\hline & & & partial \\
\hline
\end{tabular}




\begin{tabular}{|c|c|c|c|}
\hline Ensemble Pep & Gene & Transcript & \% identity in KD \\
\hline & & & 97,7 \\
\hline & & & 100 \\
\hline & & & 100 \\
\hline & & & 96,7 \\
\hline \multirow[t]{9}{*}{ ENSBTAP00000015459 } & ENSBTAG00000011639 & ENSBTAT00000015459 & 96,6 \\
\hline & & & 99,3 \\
\hline & & & 98,5 \\
\hline & & & 100 \\
\hline & & & 98,1 \\
\hline & & & 91,7 \\
\hline & & & 100 \\
\hline & & & 96,6 \\
\hline & & & 84,8 \\
\hline \multirow[t]{4}{*}{ ENSBTAP00000001500 } & ENSBTAG00000001126 & ENSBTAT00000001500 & 98,9 \\
\hline & & & 93,2 \\
\hline & & & 91,3 \\
\hline & & & 88,7 \\
\hline \multirow[t]{4}{*}{ ENSBTAP00000007991 } & & & 97,8 \\
\hline & & & 99,3 \\
\hline & & & 97,8 \\
\hline & & & 100 \\
\hline \multirow[t]{10}{*}{ ENSBTAP00000018430 } & ENSBTAG00000013876 & ENSBTAT00000018430 & 92,4 \\
\hline & & & 98,7 \\
\hline & & & 96,4 \\
\hline & & & 99,7 \\
\hline & & & 99,3 \\
\hline & & & 99,6 \\
\hline & & & 100 \\
\hline & & & 89,9 \\
\hline & & & 98,8 \\
\hline & & & 100 \\
\hline ENSBTAP00000024029 & ENSBTAG00000018049 & ENSBTAT00000024029 & 99,6 \\
\hline \multirow[t]{10}{*}{ ENSBTAP00000023654 } & ENSBTAG00000017788 & ENSBTAT00000023654 & 99,2 \\
\hline & & & 99,6 \\
\hline & & & 99,2 \\
\hline & & & 88,6 \\
\hline & & & 99,6 \\
\hline & & & 98,5 \\
\hline & & & 98,4 \\
\hline & & & 100 \\
\hline & & & 99,2 \\
\hline & & & 99,2 \\
\hline \multirow[t]{3}{*}{ ENSBTAP00000045293 } & ENSBTAG00000033983 & ENSBTAT00000048212 & 99,2 \\
\hline & & & 94,2 \\
\hline & & & 96,3 \\
\hline \multirow[t]{2}{*}{ ENSBTAP00000020910 } & & & 97,4 \\
\hline & & & 100 \\
\hline ENSBTAP00000043326 & ENSBTAG00000014351 & ENSBTAT00000045990 & 98,1 \\
\hline
\end{tabular}




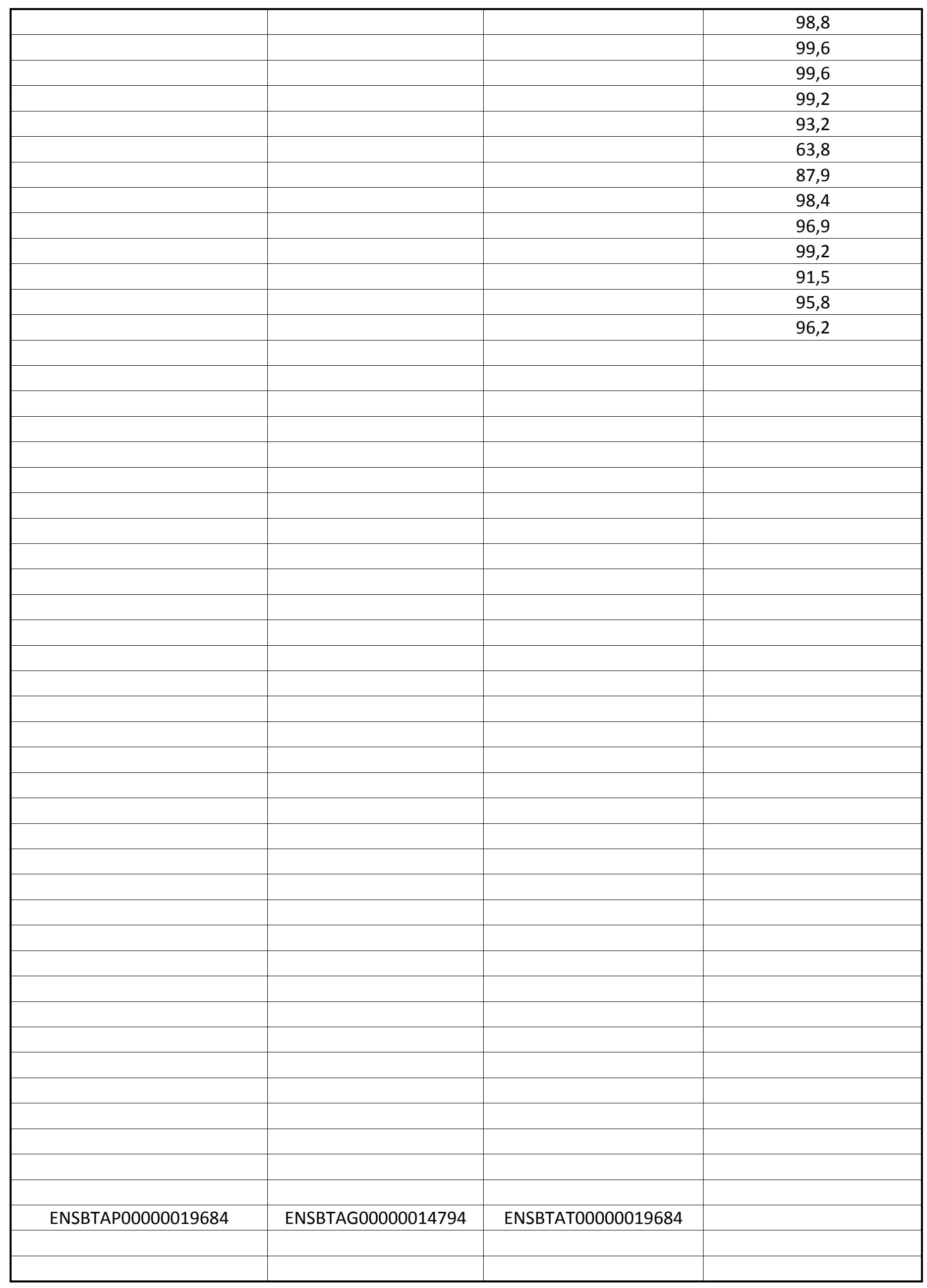




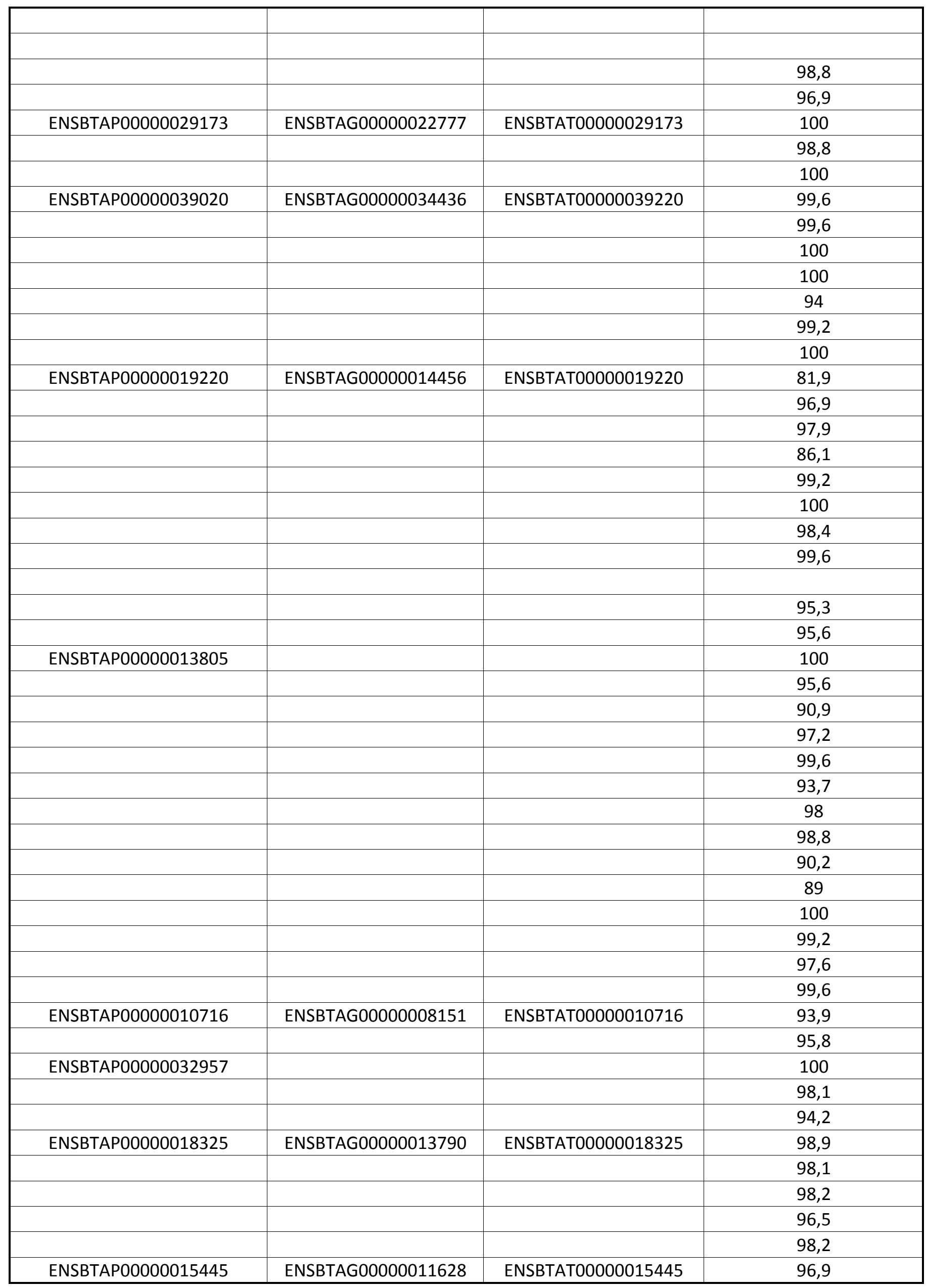




\begin{tabular}{|c|c|c|c|}
\hline ENSBTAP00000008354 & ENSBTAG00000006359 & ENSBTAT00000008354 & 93,4 \\
\hline & & & 97,7 \\
\hline \multirow[t]{5}{*}{ ENSBTAP00000007991 } & ENSBTAG00000006080 & ENSBTAT00000007991 & 98,9 \\
\hline & & & 96,5 \\
\hline & & & 94,8 \\
\hline & & & 98,1 \\
\hline & & & 98,8 \\
\hline \multirow[t]{4}{*}{ ENSBTAP00000009545 } & ENSBTAG00000007256 & ENSBTAT00000009545 & 94,5 \\
\hline & & & 96,9 \\
\hline & & & 100 \\
\hline & & & 98,4 \\
\hline \multirow[t]{5}{*}{ ENSBTAP00000009942 } & ENSBTAG00000007556 & ENSBTAT00000009942 & 99,6 \\
\hline & & & 100 \\
\hline & & & 95,5 \\
\hline & & & 98,3 \\
\hline & & & 99,6 \\
\hline ENSBTAP00000008672 & ENSBTAG00000006601 & ENSBTAT00000008672 & 88,9 \\
\hline \multirow[t]{2}{*}{ ENSBTAP00000001378 } & ENSBTAG00000001037 & ENSBTAT00000001378 & 86,8 \\
\hline & & & 94,9 \\
\hline \multirow[t]{7}{*}{ ENSBTAP00000010653 } & ENSBTAG00000008098 & ENSBTAT00000010653 & 100 \\
\hline & & & 93,3 \\
\hline & & & 98,4 \\
\hline & & & 92,7 \\
\hline & & & 93,9 \\
\hline & & & 96,1 \\
\hline & & & 97,9 \\
\hline ENSBTAP00000015986 & ENSBTAG00000012047 & ENSBTAT00000015986 & 100 \\
\hline \multirow[t]{3}{*}{ ENSBTAP00000027857 } & ENSBTAG00000020904 & ENSBTAT00000027857 & \\
\hline & & & 100 \\
\hline & & & 100 \\
\hline \multirow[t]{19}{*}{ ENSBTAP00000024657 } & ENSBTAG00000018524 & ENSBTAT00000024657 & 99,2 \\
\hline & & & 99,2 \\
\hline & & & 99,2 \\
\hline & & & 99,6 \\
\hline & & & 99,6 \\
\hline & & & 95,5 \\
\hline & & & 90,9 \\
\hline & & & 82,5 \\
\hline & & & 98,9 \\
\hline & & & 98,6 \\
\hline & & & 91,2 \\
\hline & & & 96,1 \\
\hline & & & 97,9 \\
\hline & & & 99 \\
\hline & & & 99,6 \\
\hline & & & 97,9 \\
\hline & & & 100 \\
\hline & & & 94,3 \\
\hline & & & 98,3 \\
\hline
\end{tabular}




\begin{tabular}{|c|c|c|c|}
\hline & & & 99,7 \\
\hline & & & 99,3 \\
\hline & & & 97,9 \\
\hline & & & 98,6 \\
\hline & & & 97,9 \\
\hline & & & 92,6 \\
\hline & & & 100 \\
\hline & & & 93 \\
\hline & & & 92,6 \\
\hline & & & 96,1 \\
\hline & & & 96,1 \\
\hline & & & 95,8 \\
\hline & & & 95,8 \\
\hline & & & 89,4 \\
\hline & & & 97,2 \\
\hline & & & 100 \\
\hline \multirow[t]{2}{*}{ ENSBTAP00000000016 } & ENSBTAG00000000013 & ENSBTAT00000000016 & 99,7 \\
\hline & & & 98,4 \\
\hline ENSBTAP00000026195 & ENSBTAG00000032234 & ENSBTAT00000026195 & 99,7 \\
\hline \multirow[t]{9}{*}{ ENSBTAP00000026189 } & ENSBTAG00000019652 & ENSBTAT00000026189 & 99,7 \\
\hline & & & 100 \\
\hline & & & 96,8 \\
\hline & & & 93,9 \\
\hline & & & 99,1 \\
\hline & & & 99,7 \\
\hline & & & 98,2 \\
\hline & & & 98,3 \\
\hline & & & 100 \\
\hline \multirow[t]{9}{*}{ ENSBTAP00000015200 } & ENSBTAG00000011437 & ENSBTAT00000015200 & 98,2 \\
\hline & & & 95,6 \\
\hline & & & 98,6 \\
\hline & & & 100 \\
\hline & & & 99,7 \\
\hline & & & 97 \\
\hline & & & 99 \\
\hline & & & 80 \\
\hline & & & 99,7 \\
\hline \multirow[t]{9}{*}{ ENSBTAP00000000744 } & ENSBTAG00000000570 & ENSBTAT00000000744 & 99,7 \\
\hline & & & 100 \\
\hline & & & 100 \\
\hline & & & 100 \\
\hline & & & 89,8 \\
\hline & & & 95,4 \\
\hline & & & 94,7 \\
\hline & & & 98,2 \\
\hline & & & 99,3 \\
\hline \multirow[t]{4}{*}{ ENSBTAP00000032957 } & ENSBTAG00000012114 & ENSBTAT00000033031 & 91,8 \\
\hline & & & 91,4 \\
\hline & & & 95,3 \\
\hline & & & 96,5 \\
\hline
\end{tabular}




\begin{tabular}{|c|c|c|c|}
\hline & & & 97,2 \\
\hline & & & 96 \\
\hline & & & 86,9 \\
\hline & & & \\
\hline & & & 87,4 \\
\hline \multirow[t]{10}{*}{ ENSBTAP00000023740 } & ENSBTAG00000017860 & ENSBTAT00000023740 & 89,8 \\
\hline & & & 91,8 \\
\hline & & & 89,7 \\
\hline & & & 98,9 \\
\hline & & & 89,7 \\
\hline & & & 100 \\
\hline & & & 99,3 \\
\hline & & & 85,7 \\
\hline & & & 98,5 \\
\hline & & & 97,8 \\
\hline \multirow[t]{6}{*}{ ENSBTAP00000009441 } & ENSBTAG00000007173 & ENSBTAT00000009441 & 95,3 \\
\hline & & & 100 \\
\hline & & & 97,3 \\
\hline & & & 90,5 \\
\hline & & & 71,5 \\
\hline & & & 99,6 \\
\hline \multirow[t]{2}{*}{ ENSBTAP00000017759 } & ENSBTAG00000013347 & ENSBTAT00000017759 & 86,3 \\
\hline & & & 93,5 \\
\hline ENSBTAP00000020910 & ENSBTAG00000016551 & ENSBTAT00000020910 & 94,1 \\
\hline \multirow[t]{5}{*}{ ENSBTAP00000018430 } & & & 96,1 \\
\hline & & & 94,7 \\
\hline & & & 91,5 \\
\hline & & & 97,3 \\
\hline & & & 90,6 \\
\hline ENSBTAP00000002122 & ENSBTAG00000001618 & ENSBTAT00000002122 & 97,7 \\
\hline \multirow[t]{5}{*}{ ENSBTAP00000025471 } & & & 91 \\
\hline & & & 98,8 \\
\hline & & & 98,4 \\
\hline & & & 96,9 \\
\hline & & & 99,6 \\
\hline \multirow[t]{3}{*}{ ENSBTAP00000029056 } & ENSBTAG00000021798 & ENSBTAT00000029056 & 98,9 \\
\hline & & & 80,7 \\
\hline & & & 89,3 \\
\hline \multirow[t]{5}{*}{ ENSBTAP00000013238 } & ENSBTAG00000010030 & ENSBTAT00000013238 & 85,8 \\
\hline & & & 83,1 \\
\hline & & & 78,3 \\
\hline & & & 99,7 \\
\hline & & & 99,4 \\
\hline \multirow[t]{4}{*}{ ENSBTAP00000018603 } & ENSBTAG00000014000 & ENSBTAT00000018603 & 84,4 \\
\hline & & & 100 \\
\hline & & & 100 \\
\hline & & & 92,4 \\
\hline \multirow[t]{3}{*}{ ENSBTAP00000035173 } & ENSBTAG00000010979 & ENSBTAT00000035295 & 99,6 \\
\hline & & & 69,3 \\
\hline & & & 76 \\
\hline
\end{tabular}




\begin{tabular}{|c|c|c|c|}
\hline & & & 80,9 \\
\hline & & & 89,9 \\
\hline & & & 97,4 \\
\hline & & & 97,3 \\
\hline & & & 86 \\
\hline & & & 88,2 \\
\hline & & & 77,8 \\
\hline & & & 70,1 \\
\hline & & & 96 \\
\hline & & & 100 \\
\hline & & & 95,3 \\
\hline \multirow[t]{2}{*}{ ENSBTAP00000038069 } & ENSBTAG00000003819 & ENSBTAT00000038254 & 97,2 \\
\hline & & & 95,7 \\
\hline \multirow[t]{4}{*}{ ENSBTAP00000038339 } & ENSBTAG00000020758 & ENSBTAT00000038526 & 99 \\
\hline & & & 84,2 \\
\hline & & & 98,8 \\
\hline & & & 89,5 \\
\hline ENSBTAP00000042949 & ENSBTAG00000014409 & ENSBTAT00000045570 & 98,2 \\
\hline \multirow[t]{12}{*}{ ENSBTAP00000040258 } & ENSBTAG00000000785 & ENSBTAT00000042622 & 99,6 \\
\hline & & & 91,7 \\
\hline & & & 95,1 \\
\hline & & & 98,8 \\
\hline & & & 98,9 \\
\hline & & & 97,7 \\
\hline & & & 98,4 \\
\hline & & & 85,9 \\
\hline & & & 97,5 \\
\hline & & & 94 \\
\hline & & & 98,9 \\
\hline & & & 81,1 \\
\hline ENSBTAP00000012303 & ENSBTAG00000009346 & ENSBTAT00000012303 & 100 \\
\hline \multirow[t]{7}{*}{ ENSBTAP00000019230 } & ENSBTAG00000014463 & ENSBTAT00000019230 & 99,6 \\
\hline & & & 99,2 \\
\hline & & & 98,1 \\
\hline & & & 96,3 \\
\hline & & & 99,6 \\
\hline & & & 87,2 \\
\hline & & & 92,1 \\
\hline ENSBTAP00000000463 & ENSBTAG00000000357 & ENSBTAT00000000463 & 94,5 \\
\hline ENSBTAP00000012605 & ENSBTAG00000009578 & ENSBTAT00000012605 & 99,3 \\
\hline \multirow[t]{2}{*}{ ENSBTAP00000026780 } & ENSBTAG00000020102 & ENSBTAT00000026780 & 96,9 \\
\hline & & & 100 \\
\hline \multirow[t]{7}{*}{ ENSBTAP00000016792 } & ENSBTAG00000012647 & ENSBTAT00000016792 & 98,1 \\
\hline & & & 99,2 \\
\hline & & & 96,9 \\
\hline & & & 94,9 \\
\hline & & & 95,5 \\
\hline & & & 98,9 \\
\hline & & & 100 \\
\hline
\end{tabular}




\begin{tabular}{|c|c|c|c|}
\hline & & & 99,2 \\
\hline & & & 98,1 \\
\hline & & & 99,6 \\
\hline & & & 100 \\
\hline & & & 97,4 \\
\hline & & & 100 \\
\hline ENSBTAP00000013549 & ENSBTAG00000010254 & ENSBTAT00000013549 & 99,6 \\
\hline \multirow[t]{3}{*}{ ENSBTAP00000015051 } & ENSBTAG00000011322 & ENSBTAT00000015051 & 97,6 \\
\hline & & & 99,2 \\
\hline & & & 99,6 \\
\hline \multirow[t]{2}{*}{ ENSBTAP00000042917 } & ENSBTAG00000010639 & ENSBTAT00000045536 & 94,8 \\
\hline & & & 98,1 \\
\hline \multirow[t]{11}{*}{ ENSBTAP00000019139 } & ENSBTAG00000014393 & ENSBTAT00000019139 & 99,6 \\
\hline & & & 99,2 \\
\hline & & & 100 \\
\hline & & & 99,2 \\
\hline & & & 97,2 \\
\hline & & & 98,4 \\
\hline & & & 96,9 \\
\hline & & & 98,5 \\
\hline & & & 95,2 \\
\hline & & & 97,4 \\
\hline & & & 99,6 \\
\hline \multirow[t]{16}{*}{ ENSBTAP00000046377 } & ENSBTAG00000035028 & ENSBTAT00000049489 & 100 \\
\hline & & & 97,2 \\
\hline & & & 99,6 \\
\hline & & & 99,2 \\
\hline & & & 99,2 \\
\hline & & & 100 \\
\hline & & & 97 \\
\hline & & & 99,2 \\
\hline & & & 100 \\
\hline & & & 93,3 \\
\hline & & & 98,9 \\
\hline & & & 100 \\
\hline & & & 98,4 \\
\hline & & & 91,8 \\
\hline & & & 99,2 \\
\hline & & & 100 \\
\hline \multirow[t]{2}{*}{ ENSBTAP00000040257 } & ENSBTAG00000030201 & ENSBTAT00000042621 & 98,5 \\
\hline & & & 90,1 \\
\hline \multirow[t]{2}{*}{ ENSBTAP00000018713 } & ENSBTAG00000014064 & ENSBTAT00000018713 & 98,5 \\
\hline & & & 97,8 \\
\hline \multirow[t]{4}{*}{ ENSBTAP00000020866 } & ENSBTAG00000015713 & ENSBTAT00000020866 & 94,7 \\
\hline & & & 98,1 \\
\hline & & & 97,4 \\
\hline & & & 99,2 \\
\hline \multirow[t]{2}{*}{ ENSBTAP00000021631 } & ENSBTAG00000016260 & ENSBTAT00000021631 & 96,3 \\
\hline & & & 96,6 \\
\hline ENSBTAP00000013808 & ENSBTAG00000010460 & ENSBTAT00000013808 & 97,2 \\
\hline
\end{tabular}




\begin{tabular}{|c|c|c|c|}
\hline & & & 98,1 \\
\hline & & & 98,8 \\
\hline & & & 98,8 \\
\hline & & & 94,6 \\
\hline \multirow[t]{4}{*}{ ENSBTAP00000013805 } & ENSBTAG00000010457 & ENSBTAT00000013805 & 93,1 \\
\hline & & & 92,2 \\
\hline & & & 95,7 \\
\hline & & & 100 \\
\hline ENSBTAP00000013238 & & & 99,6 \\
\hline \multirow[t]{5}{*}{ ENSBTAP00000025471 } & ENSBTAG00000019134 & ENSBTAT00000025471 & 98,8 \\
\hline & & & 99,3 \\
\hline & & & 95,7 \\
\hline & & & 98,1 \\
\hline & & & 89,7 \\
\hline ENSBTAP00000014411 & ENSBTAG00000010855 & ENSBTAT00000014411 & 100 \\
\hline \multirow[t]{6}{*}{ ENSBTAP00000013449 } & ENSBTAG00000015670 & ENSBTAT00000013449 & 100 \\
\hline & & & 99,6 \\
\hline & & & 92,3 \\
\hline & & & 97,6 \\
\hline & & & 97,3 \\
\hline & & & 93,3 \\
\hline \multicolumn{4}{|l|}{ ENSBTAP00000018603 } \\
\hline & & & 98,4 \\
\hline & & & 99,3 \\
\hline & & & 99,3 \\
\hline & & & 98,6 \\
\hline & & & 98,6 \\
\hline & & & 98,9 \\
\hline & & & 98,9 \\
\hline & & & 95,6 \\
\hline & & & 98,2 \\
\hline & & & 97,8 \\
\hline & & & 94 \\
\hline & & & 85,7 \\
\hline & & & 93,4 \\
\hline & & & 94,6 \\
\hline \multirow[t]{9}{*}{ ENSBTAP00000012838 } & ENSBTAG00000009736 & ENSBTAT00000012838 & 98,8 \\
\hline & & & 97,7 \\
\hline & & & 91,7 \\
\hline & & & 95 \\
\hline & & & 96,4 \\
\hline & & & 97,3 \\
\hline & & & 99,1 \\
\hline & & & 99,4 \\
\hline & & & 98,6 \\
\hline \multirow[t]{4}{*}{ ENSBTAP00000026519 } & ENSBTAG00000019907 & ENSBTAT00000026519 & 94,4 \\
\hline & & & 99,3 \\
\hline & & & 94,5 \\
\hline & & & 99,3 \\
\hline
\end{tabular}




\begin{tabular}{|c|c|c|c|}
\hline \multirow{9}{*}{ ENSBTAP00000012197 } & ENSBTAG00000009258 & ENSBTAT00000012197 & \\
\hline & & & 94,4 \\
\hline & & & 85 \\
\hline & & & 92,4 \\
\hline & & & 96,1 \\
\hline & & & 99,6 \\
\hline & & & 96,8 \\
\hline & & & 97,2 \\
\hline & & & 99,6 \\
\hline \multirow[t]{5}{*}{ ENSBTAP00000022534 } & ENSBTAG00000016943 & ENSBTAT00000022534 & 99,2 \\
\hline & & & 84,1 \\
\hline & & & 100 \\
\hline & & & 98 \\
\hline & & & 96,4 \\
\hline \multirow[t]{10}{*}{ ENSBTAP00000022347 } & ENSBTAG00000016799 & ENSBTAT00000022347 & 94 \\
\hline & & & 99,2 \\
\hline & & & 96,8 \\
\hline & & & 96,8 \\
\hline & & & 89,3 \\
\hline & & & 99,3 \\
\hline & & & 100 \\
\hline & & & 79,4 \\
\hline & & & 98,9 \\
\hline & & & 100 \\
\hline \multirow[t]{12}{*}{ ENSBTAP00000015530 } & ENSBTAG00000010408 & ENSBTAT00000015530 & 100 \\
\hline & & & 95,7 \\
\hline & & & 92,3 \\
\hline & & & 96 \\
\hline & & & 87,4 \\
\hline & & & 82,8 \\
\hline & & & 89,2 \\
\hline & & & 94,1 \\
\hline & & & 99,2 \\
\hline & & & 95,9 \\
\hline & & & 96,5 \\
\hline & & & 85,4 \\
\hline \multirow[t]{3}{*}{ ENSBTAP00000030935 } & ENSBTAG00000003325 & ENSBTAT00000030970 & 95,1 \\
\hline & & & 98 \\
\hline & & & 97,7 \\
\hline ENSBTAP00000010292 & ENSBTAG00000007825 & ENSBTAT00000010292 & 99,2 \\
\hline \multirow[t]{10}{*}{ ENSBTAP00000004466 } & ENSBTAG00000030977 & ENSBTAT00000004466 & 99,6 \\
\hline & & & 98,8 \\
\hline & & & 98,5 \\
\hline & & & 99,2 \\
\hline & & & 96,4 \\
\hline & & & 100 \\
\hline & & & 100 \\
\hline & & & 99,2 \\
\hline & & & 100 \\
\hline & & & 97,7 \\
\hline
\end{tabular}




\begin{tabular}{|c|c|c|c|}
\hline & & & 99,6 \\
\hline & & & 99,6 \\
\hline & & & 96,2 \\
\hline ENSBTAP00000009766 & ENSBTAG00000007428 & ENSBTAT00000009766 & 77,3 \\
\hline & & & 94,8 \\
\hline ENSBTAP00000012302 & ENSBTAG00000009343 & ENSBTAT00000012302 & 71,9 \\
\hline & & & 75,3 \\
\hline & & & 98,3 \\
\hline ENSBTAP00000022500 & ENSBTAG00000016915 & ENSBTAT00000022500 & 100 \\
\hline & & & 100 \\
\hline & & & 99,3 \\
\hline ENSBTAP00000025356 & ENSBTAG00000019049 & ENSBTAT00000025356 & 99,3 \\
\hline & & & 99,7 \\
\hline & & & 99,7 \\
\hline & & & 100 \\
\hline ENSBTAP00000024657 & & & 99 \\
\hline & ENSBTAG00000012016 & ENSBTAT00000031813 & 99,7 \\
\hline & & & 99,7 \\
\hline & & & 99,2 \\
\hline
\end{tabular}




\begin{tabular}{|c|c|}
\hline \% identity in Protein & Comments \\
\hline 96,3 & \\
\hline 98,1 & \\
\hline 100 & \\
\hline 80,1 & \\
\hline 84,5 & Insertion in human \\
\hline 97 & Insertion in human \\
\hline 90,3 & Deletion in human \\
\hline 93,9 & \\
\hline 97,8 & \\
\hline 97,2 & \\
\hline 98 & \\
\hline 95,3 & \\
\hline 75,6 & \\
\hline 97,1 & \\
\hline 87,4 & Insertion/Deletion \\
\hline 85,4 & \\
\hline 83 & \\
\hline 94,6 & \\
\hline 85,9 & Insertion in human \\
\hline 87,8 & Deletion in human \\
\hline 73,6 & \\
\hline 83,8 & \\
\hline 94,9 & \\
\hline 70,5 & Insertion in human \\
\hline 99,4 & \\
\hline 99,1 & \\
\hline 99,4 & \\
\hline 98,9 & \\
\hline 85,6 & Insertion in human \\
\hline 92,3 & \\
\hline 99,1 & \\
\hline 93,1 & \\
\hline 97,8 & \\
\hline 98,4 & \\
\hline 98 & \\
\hline 87,7 & \\
\hline 99,2 & \\
\hline 98 & \\
\hline 95,9 & \\
\hline 88,5 & Insertion in human \\
\hline 97,2 & \\
\hline 95,4 & \\
\hline 97 & \\
\hline 89,1 & \\
\hline 90,9 & \\
\hline 95,7 & \\
\hline 99,4 & \\
\hline 93,4 & \\
\hline
\end{tabular}




\begin{tabular}{|c|c|}
\hline 96,7 & \\
\hline 99,9 & \\
\hline 98,9 & \\
\hline 96,1 & \\
\hline 88,5 & Insertion in human \\
\hline 72,9 & Insertion in human \\
\hline 81,9 & Insertion in human \\
\hline 96,8 & \\
\hline 95,9 & \\
\hline 98 & \\
\hline 91,8 & \\
\hline 84,1 & \\
\hline 89,5 & Deletion in human \\
\hline 94,9 & \\
\hline 95,1 & \\
\hline 87,3 & \\
\hline 87 & \\
\hline 87,6 & \\
\hline 83,8 & \\
\hline 78,4 & \\
\hline 96 & \\
\hline 83,8 & Deletion in human \\
\hline 91,2 & \\
\hline 73,8 & Insertion in human \\
\hline 57,5 & Insertion/Deletion \\
\hline 74,4 & Deletion in human \\
\hline 89,1 & Insertion in human \\
\hline 97,4 & \\
\hline 93,5 & \\
\hline 96,4 & Deletion in human \\
\hline 79,2 & Insertion in human \\
\hline 90,7 & Insertion in human \\
\hline 79,2 & Insertion in human \\
\hline 95,9 & \\
\hline 96,4 & \\
\hline 94,7 & \\
\hline 97,4 & \\
\hline 96,4 & \\
\hline 90,8 & Insertion in human \\
\hline 89,8 & \\
\hline 93,4 & \\
\hline 77,8 & \\
\hline 99,5 & \\
\hline 98,7 & \\
\hline 97,8 & \\
\hline 88,4 & \\
\hline 87,1 & \\
\hline 95,8 & \\
\hline 97,1 & Deletion in human \\
\hline 47,7 & Insertion/Deletion \\
\hline
\end{tabular}




\begin{tabular}{|c|c|}
\hline 95,5 & Insertion in human \\
\hline 49,3 & Insertion/Deletion \\
\hline \multicolumn{2}{|l|}{95,7} \\
\hline \multicolumn{2}{|l|}{91} \\
\hline 85,3 & Insertion/Deletion \\
\hline \multicolumn{2}{|l|}{91,4} \\
\hline \multicolumn{2}{|l|}{99,6} \\
\hline \multicolumn{2}{|l|}{98,9} \\
\hline \multicolumn{2}{|l|}{99,4} \\
\hline 95,6 & Insertion in human \\
\hline 81,4 & Insertion in human \\
\hline 96,5 & Deletion in human \\
\hline \multicolumn{2}{|l|}{97,6} \\
\hline 99,1 & Deletion in human \\
\hline 90,4 & Deletion in human \\
\hline \multicolumn{2}{|l|}{95,8} \\
\hline 85,7 & Insertion in human \\
\hline 84,6 & Deletion in human \\
\hline \multicolumn{2}{|l|}{95,5} \\
\hline \multicolumn{2}{|l|}{97,6} \\
\hline 86,6 & Deletion in human \\
\hline \multicolumn{2}{|r|}{ Insertion in human } \\
\hline \multicolumn{2}{|l|}{89,4} \\
\hline \multicolumn{2}{|l|}{93,8} \\
\hline \multicolumn{2}{|l|}{93,7} \\
\hline 80 & Insertion/Deletion \\
\hline 64 & Deletion in human \\
\hline \multicolumn{2}{|l|}{87,9} \\
\hline \multicolumn{2}{|r|}{ Insertion in human } \\
\hline \multicolumn{2}{|r|}{ Insertion in human } \\
\hline \multicolumn{2}{|r|}{ Insertion in human } \\
\hline \multicolumn{2}{|l|}{96,1} \\
\hline \multicolumn{2}{|l|}{75} \\
\hline \multicolumn{2}{|l|}{93,3} \\
\hline 97,5 & Deletion in human \\
\hline \multicolumn{2}{|l|}{94,8} \\
\hline 93,2 & Deletion in human \\
\hline \multicolumn{2}{|l|}{94,9} \\
\hline \multicolumn{2}{|l|}{89,4} \\
\hline 93 & \\
\hline 89,4 & \\
\hline 95,6 & Insertion/Deletion \\
\hline 76,5 & Deletion in human \\
\hline 92,8 & \\
\hline 95,9 & \\
\hline 96,6 & \\
\hline 91,2 & \\
\hline 95,1 & Deletion in human \\
\hline 66,5 & Insertion in human \\
\hline
\end{tabular}




\begin{tabular}{|c|c|}
\hline 80,9 & \\
\hline 80,6 & Deletion in human \\
\hline 74,4 & Insertion in human \\
\hline 94,3 & \\
\hline 96,3 & \\
\hline 98,7 & \\
\hline 84,1 & \\
\hline 94,8 & \\
\hline 93,5 & Insertion in human \\
\hline 94,5 & \\
\hline 97,6 & \\
\hline 97,9 & \\
\hline 86 & Insertion in human \\
\hline 96,8 & \\
\hline 99,1 & \\
\hline 81,3 & \\
\hline 66,9 & Insertion in human \\
\hline 91,7 & \\
\hline 97,1 & Deletion in human \\
\hline 80,2 & Insertion in human \\
\hline 98,2 & \\
\hline 84,1 & \\
\hline 90,8 & \\
\hline 95,9 & \\
\hline 85,3 & Deletion in human \\
\hline 99,7 & \\
\hline 97,6 & \\
\hline 98,7 & Deletion in human \\
\hline 96 & Deletion in human \\
\hline 95,7 & \\
\hline 98,4 & \\
\hline 88,6 & \\
\hline 91,2 & \\
\hline 90,9 & \\
\hline 73,7 & \\
\hline 72,8 & Insertion in human \\
\hline 98,7 & \\
\hline 98,7 & \\
\hline 88,5 & \\
\hline 93,1 & \\
\hline 97 & \\
\hline 97,5 & \\
\hline 99,7 & \\
\hline 96,8 & \\
\hline 98 & \\
\hline 93,3 & \\
\hline 98,4 & \\
\hline
\end{tabular}




\begin{tabular}{|c|c|}
\hline 96,1 & \\
\hline 93,8 & \\
\hline 93,6 & Insertion in human \\
\hline 98 & \\
\hline 98,5 & \\
\hline 86,3 & Insertion in human \\
\hline 98,2 & \\
\hline 90,4 & \\
\hline 92,5 & \\
\hline 89,7 & Insertion in human \\
\hline 76,8 & Deletion in human \\
\hline 86,3 & Deletion in human \\
\hline 93,1 & Insertion in human \\
\hline 84,7 & Insertion in human \\
\hline 93,2 & \\
\hline 98,6 & \\
\hline 98,2 & \\
\hline 90,9 & Insertion in human \\
\hline 98,4 & Deletion in human \\
\hline 98,7 & \\
\hline 98,2 & \\
\hline 92,5 & \\
\hline 80,5 & Insertion in human \\
\hline 98 & \\
\hline 96,7 & \\
\hline 94,5 & \\
\hline 76,2 & Deletion in human \\
\hline 96,9 & \\
\hline 92,9 & \\
\hline 95,6 & Deletion in human \\
\hline 93,1 & Insertion in human \\
\hline 99,7 & \\
\hline 97,6 & \\
\hline 96,1 & Insertion in human \\
\hline 88,4 & Insertion in human \\
\hline 67,4 & Insertion in human \\
\hline 99,2 & Deletion in human \\
\hline 99,5 & \\
\hline 99,2 & Insertion/Deletion \\
\hline 100 & \\
\hline 100 & \\
\hline 90,3 & Insertion in human \\
\hline 94,8 & \\
\hline 92,9 & \\
\hline 91,5 & \\
\hline 87 & \\
\hline 79,8 & \\
\hline 89,9 & Deletion in human \\
\hline 95,6 & \\
\hline 96,5 & \\
\hline
\end{tabular}




\begin{tabular}{|c|c|}
\hline 88,9 & \\
\hline 93 & \\
\hline 80,6 & \\
\hline 78,6 & \\
\hline 88,8 & \\
\hline 88,2 & \\
\hline 91,1 & Insertion in human \\
\hline 90,2 & Insertion in human \\
\hline 89,1 & \\
\hline 99,7 & \\
\hline 99,1 & \\
\hline 70,9 & Insertion in human \\
\hline 96,5 & Insertion in human \\
\hline 93,3 & \\
\hline 88 & \\
\hline 97,1 & \\
\hline 93,3 & \\
\hline 85,4 & \\
\hline 68,6 & Insertion in human \\
\hline 94,4 & Insertion/Deletion \\
\hline 81,6 & Insertion/Deletion \\
\hline 78,6 & Insertion in human \\
\hline 91,8 & \\
\hline 89,9 & Insertion in human \\
\hline 90,2 & \\
\hline 80,1 & Insertion in human \\
\hline 92,8 & \\
\hline 84,4 & Insertion in human \\
\hline 76,6 & Insertion in human \\
\hline 90,4 & Insertion in human \\
\hline 95,9 & \\
\hline 98 & \\
\hline 93,2 & \\
\hline 96,1 & \\
\hline 89,5 & Insertion in human \\
\hline 77,7 & \\
\hline 82,5 & Insertion in human \\
\hline 85,4 & \\
\hline 78,5 & Deletion in human \\
\hline 90,4 & Insertion in human \\
\hline 97,3 & Insertion in human \\
\hline 99,4 & Deletion in human \\
\hline 56,2 & Insertion/Deletion \\
\hline 72,4 & Deletion in human \\
\hline 99,4 & \\
\hline 91,3 & Insertion in human \\
\hline 99,5 & \\
\hline 69,6 & \\
\hline 54,5 & Deletion in human \\
\hline
\end{tabular}




\begin{tabular}{|c|c|}
\hline 76,4 & \\
\hline 82,5 & \\
\hline 95,1 & \\
\hline 93,8 & \\
\hline 89,4 & \\
\hline 89,3 & \\
\hline 80,4 & \\
\hline 61,6 & Insertion in human \\
\hline 94,9 & \\
\hline 98,1 & \\
\hline 92,1 & \\
\hline 82,4 & Insertion in human \\
\hline 91,5 & \\
\hline 96,1 & Insertion in human \\
\hline 70,6 & Insertion/Deletion \\
\hline 94,6 & \\
\hline 94,9 & Deletion in human \\
\hline 94,7 & Insertion in human \\
\hline 96,8 & Deletion in human \\
\hline 88,9 & \\
\hline 85,5 & \\
\hline 88,2 & \\
\hline 89,6 & \\
\hline 86,8 & \\
\hline 97 & \\
\hline 83,2 & Deletion in human \\
\hline 96,6 & \\
\hline 87,5 & Insertion/Deletion \\
\hline 91,9 & \\
\hline 73 & \\
\hline 81 & \\
\hline 77,5 & \\
\hline 92,3 & \\
\hline 87 & \\
\hline 92,6 & \\
\hline 98,3 & \\
\hline 86,6 & Deletion in human \\
\hline 89,6 & \\
\hline 85,3 & \\
\hline 93,3 & \\
\hline 95,6 & \\
\hline 96,9 & \\
\hline 91,5 & Insertion in human \\
\hline 95 & Insertion in human \\
\hline 87,4 & \\
\hline 83,1 & \\
\hline 79,6 & Deletion in human \\
\hline 97,2 & \\
\hline 95,6 & Insertion in human \\
\hline
\end{tabular}




\begin{tabular}{|c|c|}
\hline 94,4 & \\
\hline 89,7 & \\
\hline 97,4 & \\
\hline 97,4 & \\
\hline 89,8 & \\
\hline 98,6 & \\
\hline 93,2 & Deletion in human \\
\hline 85,4 & \\
\hline 97,1 & \\
\hline 98,8 & Deletion in human \\
\hline 85 & \\
\hline 95,7 & Insertion in human \\
\hline 98,7 & \\
\hline 97,3 & Insertion in human \\
\hline 99,6 & \\
\hline 93,8 & \\
\hline 94 & \\
\hline 93,4 & \\
\hline 89,9 & \\
\hline 90,1 & Insertion/Deletion \\
\hline 94,9 & \\
\hline 93,5 & \\
\hline 99,7 & \\
\hline 99,2 & \\
\hline 97,6 & \\
\hline 94,7 & \\
\hline 98,6 & \\
\hline 93,2 & \\
\hline 100 & \\
\hline 97 & \\
\hline 96,3 & \\
\hline 95,9 & Deletion in human \\
\hline 89,3 & Insertion/Deletion \\
\hline 98,5 & \\
\hline 99,2 & Deletion in human \\
\hline 95,9 & \\
\hline 87,4 & \\
\hline 88,2 & Deletion in human \\
\hline 96 & \\
\hline 94,1 & Deletion in human \\
\hline 82,7 & \\
\hline 88,9 & Insertion/Deletion \\
\hline 81,2 & Deletion in human \\
\hline 81,3 & \\
\hline 91,7 & Deletion in human \\
\hline 94,1 & Deletion in human \\
\hline 98,7 & \\
\hline 93,4 & \\
\hline 90,8 & Deletion in human \\
\hline 97,2 & \\
\hline
\end{tabular}




\begin{tabular}{|c|c|}
\hline 87,6 & \\
\hline 91,3 & Deletion in human \\
\hline 93,1 & \\
\hline 96,8 & Insertion in human \\
\hline 84 & Insertion/Deletion \\
\hline 91,3 & \\
\hline 92,9 & \\
\hline 97,3 & \\
\hline 97,9 & \\
\hline 97,7 & \\
\hline 98 & Deletion in human \\
\hline 98,2 & \\
\hline 97,3 & Deletion in human \\
\hline 96,5 & \\
\hline 98,4 & Insertion in human \\
\hline 98 & \\
\hline 93,7 & Deletion in human \\
\hline 91,7 & Insertion in human \\
\hline 95,3 & Deletion in human \\
\hline 95,5 & \\
\hline 94,8 & \\
\hline 92,5 & \\
\hline 98,5 & \\
\hline 93,9 & \\
\hline 93,1 & \\
\hline 92,4 & \\
\hline 97,7 & Insertion in human \\
\hline 83,1 & Insertion in human \\
\hline 92,9 & \\
\hline 96 & \\
\hline 94,8 & \\
\hline 87,9 & \\
\hline 81,6 & \\
\hline 74,4 & \\
\hline 73,8 & \\
\hline 88 & Insertion in human \\
\hline 89,5 & \\
\hline 80,1 & \\
\hline 94 & \\
\hline 90,7 & \\
\hline 83,1 & \\
\hline 87,2 & \\
\hline 94,1 & \\
\hline 90,7 & \\
\hline 87,2 & \\
\hline 97,9 & \\
\hline 93,5 & \\
\hline 97,2 & Insertion in human \\
\hline
\end{tabular}




\begin{tabular}{|c|c|}
\hline 86,6 & Insertion in human \\
\hline 81,2 & \\
\hline 90 & \\
\hline 87 & Deletion in human \\
\hline 99,6 & \\
\hline 90,9 & \\
\hline 96,3 & \\
\hline 96,9 & \\
\hline 97 & Deletion in human \\
\hline 76,9 & \\
\hline 96,9 & \\
\hline 88,4 & Insertion in human \\
\hline 93,4 & \\
\hline 89,1 & Insertion in human \\
\hline 98,6 & \\
\hline 95,5 & \\
\hline 86,7 & \\
\hline 89 & \\
\hline 92,8 & \\
\hline 94,9 & \\
\hline 75,6 & \\
\hline 92,7 & \\
\hline 94,2 & \\
\hline 97,5 & \\
\hline 83,7 & Deletion in human \\
\hline 87,6 & Deletion in human \\
\hline 91,1 & \\
\hline 80,4 & \\
\hline 77,6 & \\
\hline 80,7 & \\
\hline 89,2 & \\
\hline 97,5 & \\
\hline 92,2 & \\
\hline 94,3 & \\
\hline 78,1 & Insertion/Deletion \\
\hline 86,1 & \\
\hline 86,3 & Insertion/Deletion \\
\hline 96,5 & \\
\hline 92,6 & Deletion in human \\
\hline 93,8 & \\
\hline 94,8 & Insertion in human \\
\hline 79 & Insertion in human \\
\hline 92,6 & \\
\hline 80,2 & Deletion in human \\
\hline 92,5 & \\
\hline 94,7 & Insertion in human \\
\hline 96,5 & \\
\hline 97,8 & Insertion/Deletion \\
\hline 89,3 & Insertion in human \\
\hline
\end{tabular}




\begin{tabular}{|c|c|}
\hline 83,5 & Insertion in human \\
\hline 94,8 & Deletion in human \\
\hline 89,9 & \\
\hline 74,4 & \\
\hline 86,7 & Insertion/Deletion \\
\hline 61,7 & Insertion in human \\
\hline 66,1 & \\
\hline 90,5 & \\
\hline 99,2 & \\
\hline 94,3 & \\
\hline 93,5 & \\
\hline 97,9 & \\
\hline 98,6 & Insertion in human \\
\hline 96,4 & \\
\hline 98,6 & \\
\hline 97,9 & Insertion in human \\
\hline 96,5 & \\
\hline 80,7 & \\
\hline 91,5 & \\
\hline 99,3 & \\
\hline 63,6 & \\
\hline & \\
\hline & \\
\hline & \\
\hline & \\
\hline & \\
\hline & \\
\hline & \\
\hline & \\
\hline & \\
\hline & \\
\hline & \\
\hline & \\
\hline & \\
\hline & \\
\hline & \\
\hline & \\
\hline & \\
\hline
\end{tabular}




\section{Figure Legend}

\section{Figure S1.}

Phylogenetic trees of bovine and human protein kinases. The phylogenetic trees were created by aligning the catalytic domain sequences of human and bovine protein kinases using the NJ method. Each empty node has a bootstrap value of 100. H, Human; B, Bovine. 


\section{AGC}

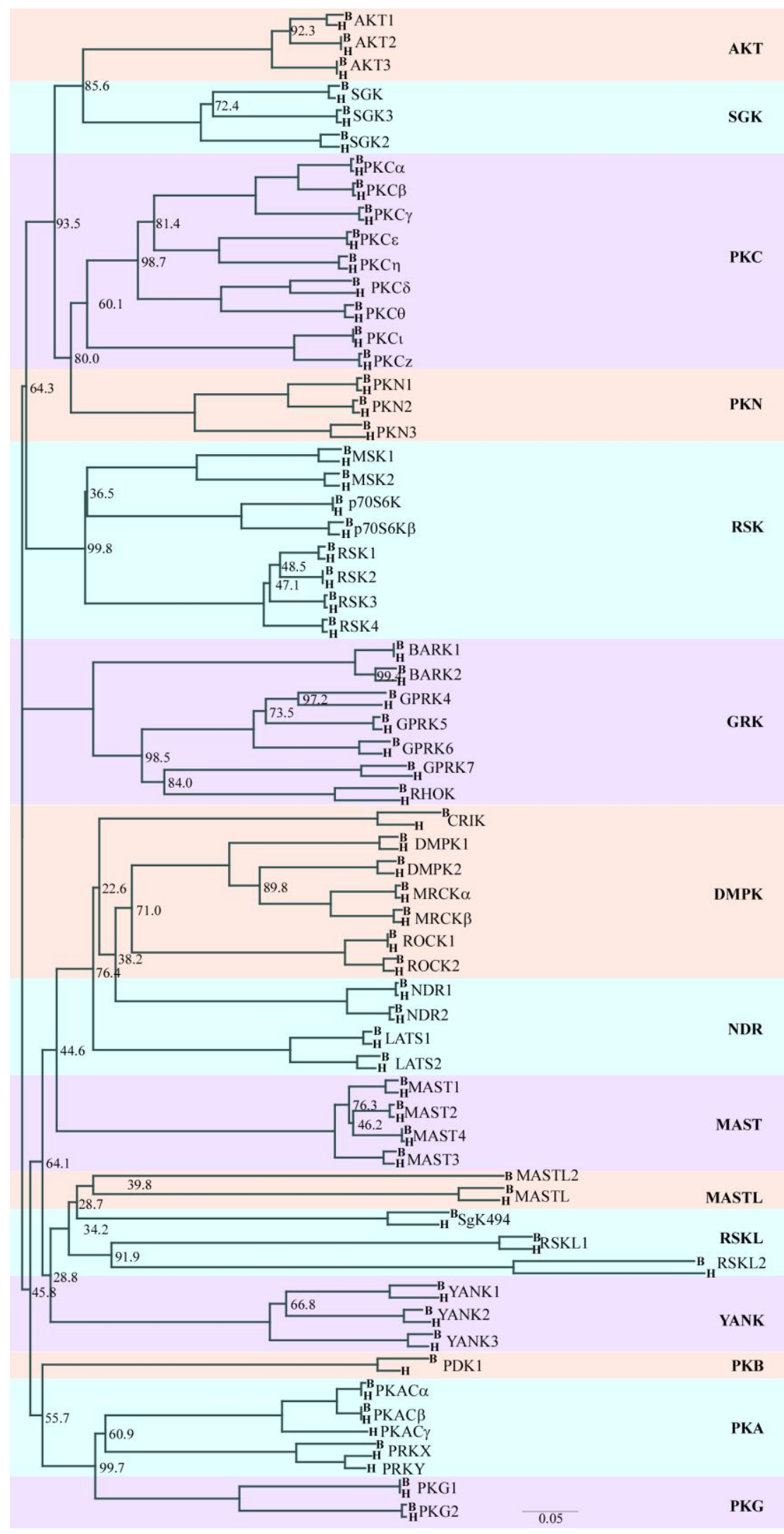




\section{CAMK}

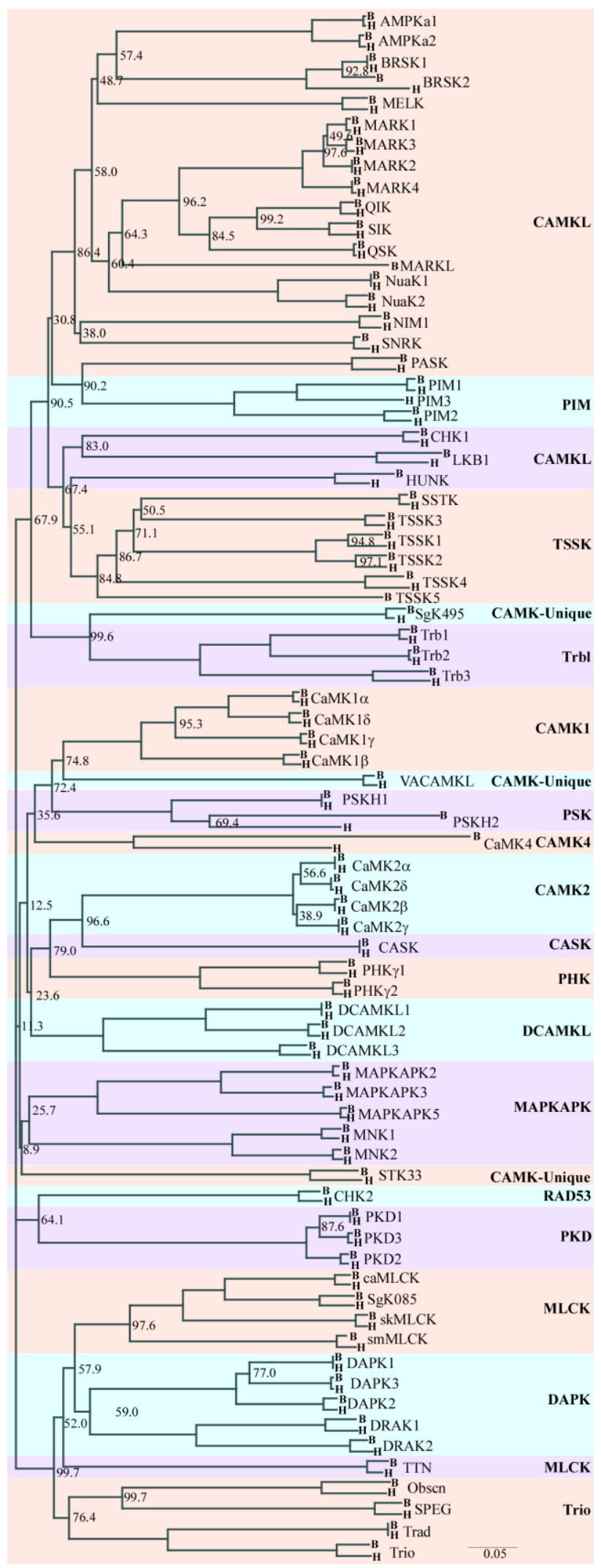




\section{CK1}

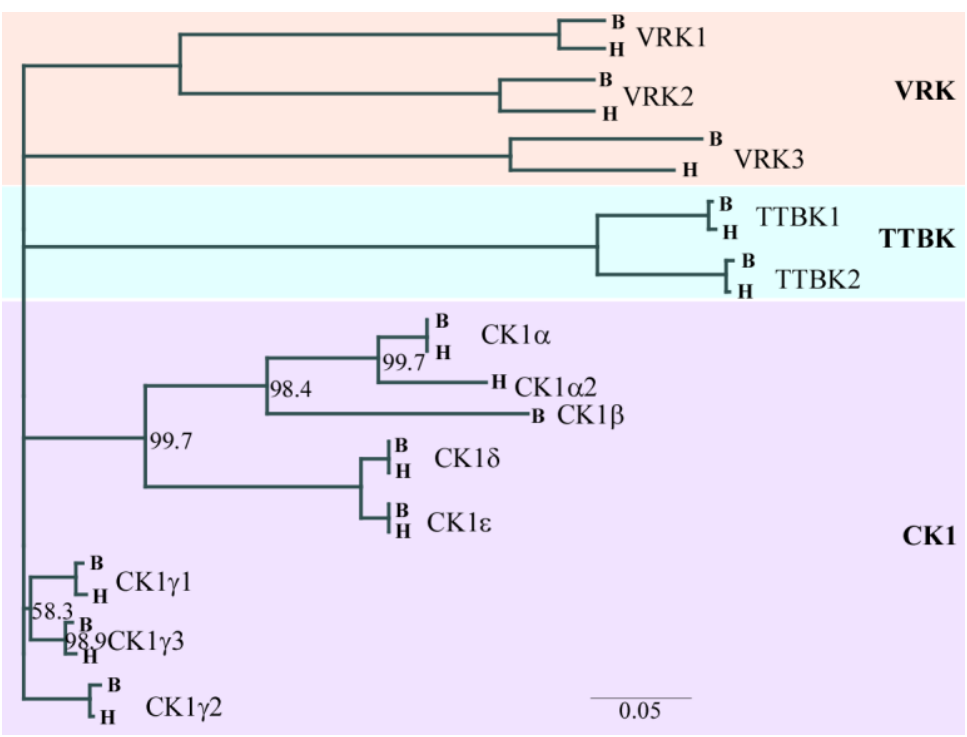




\section{CMGC}

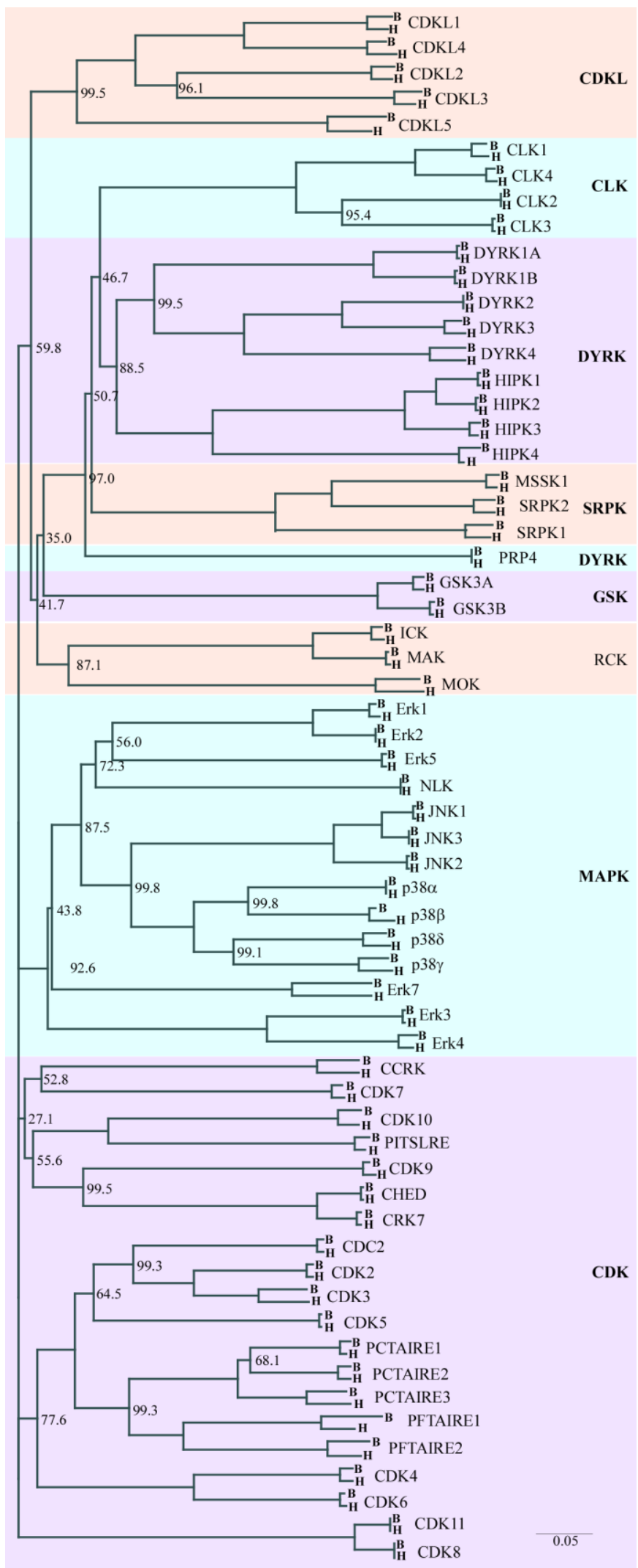




\section{Other}

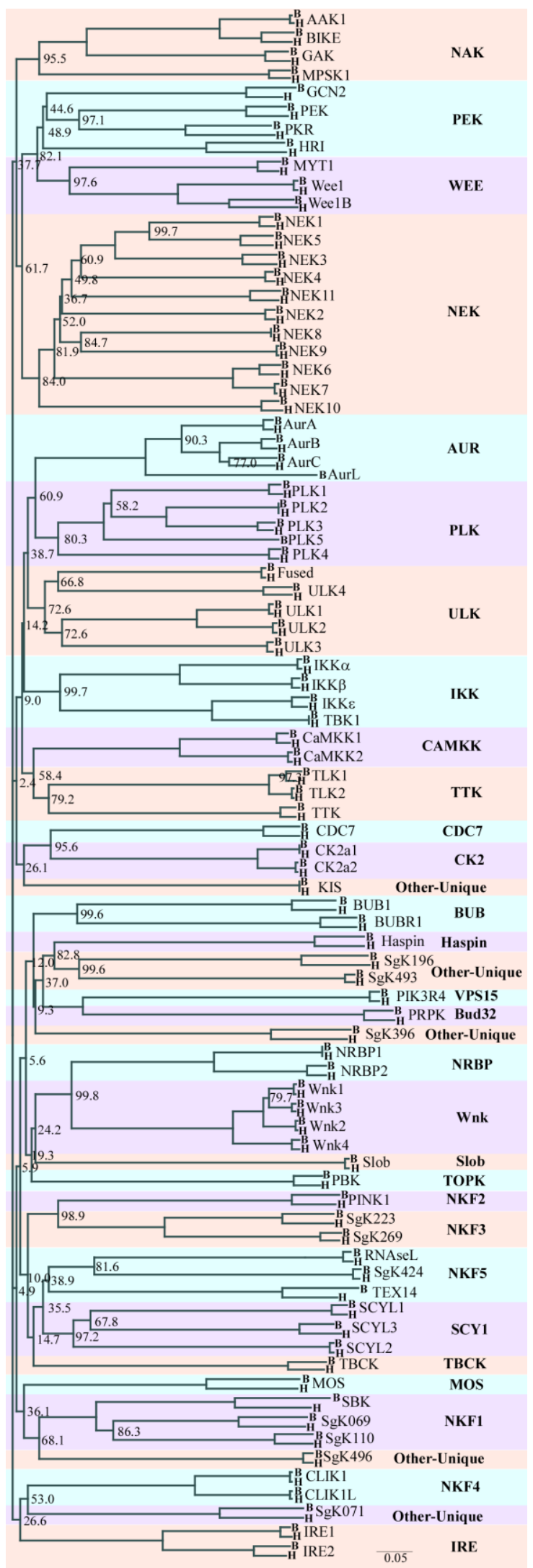




\section{RGC}

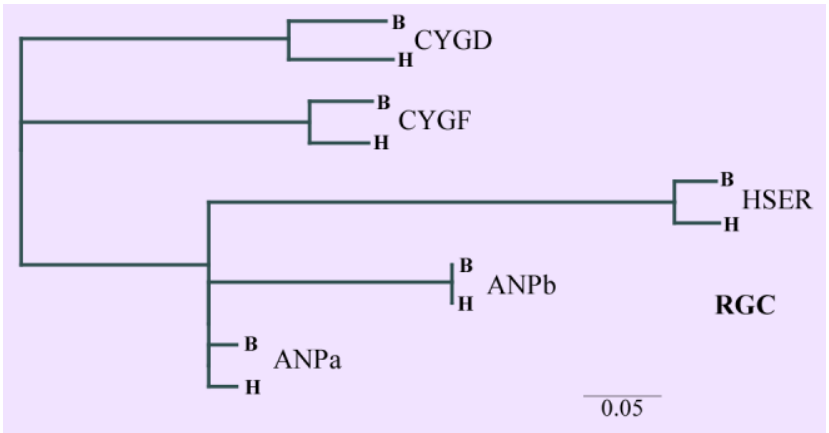




\section{STE}

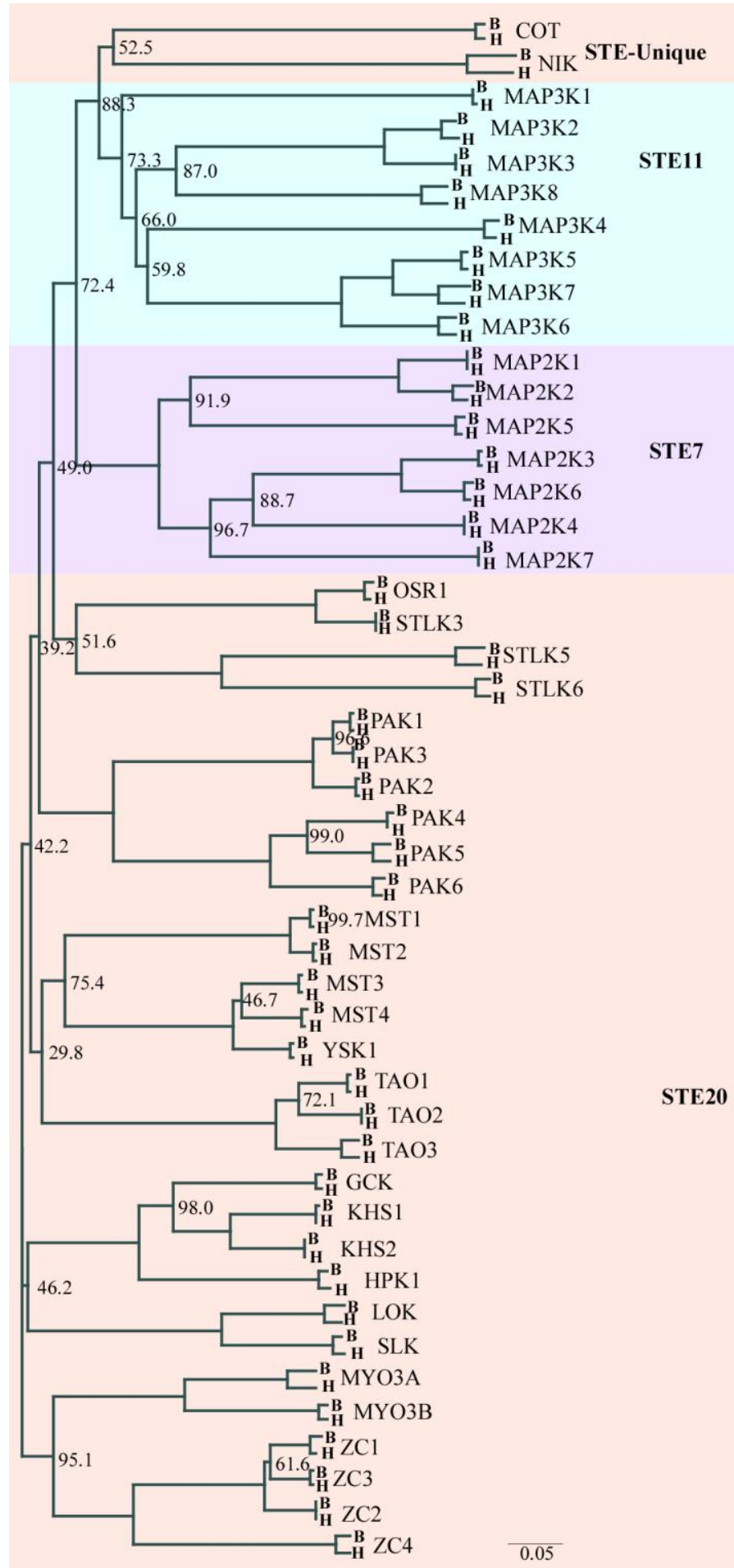




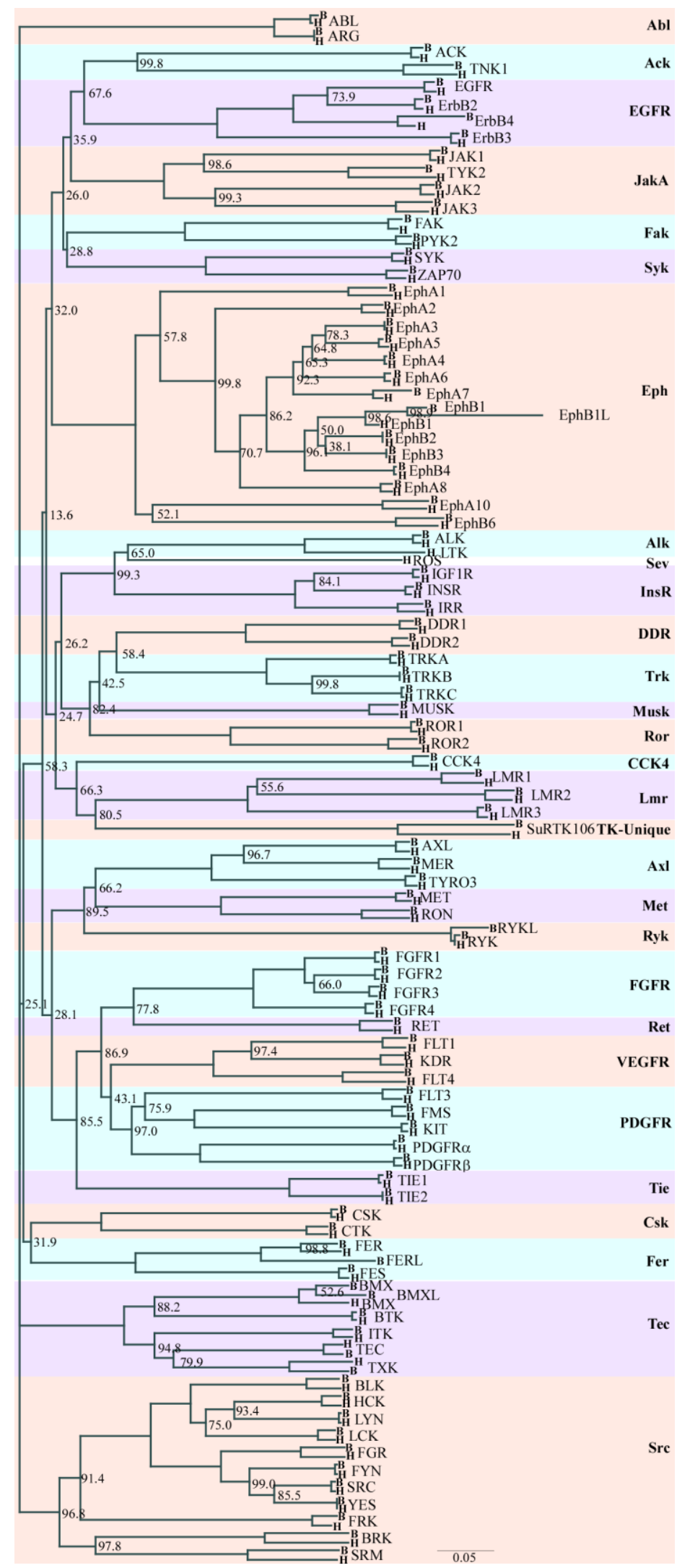


TKL

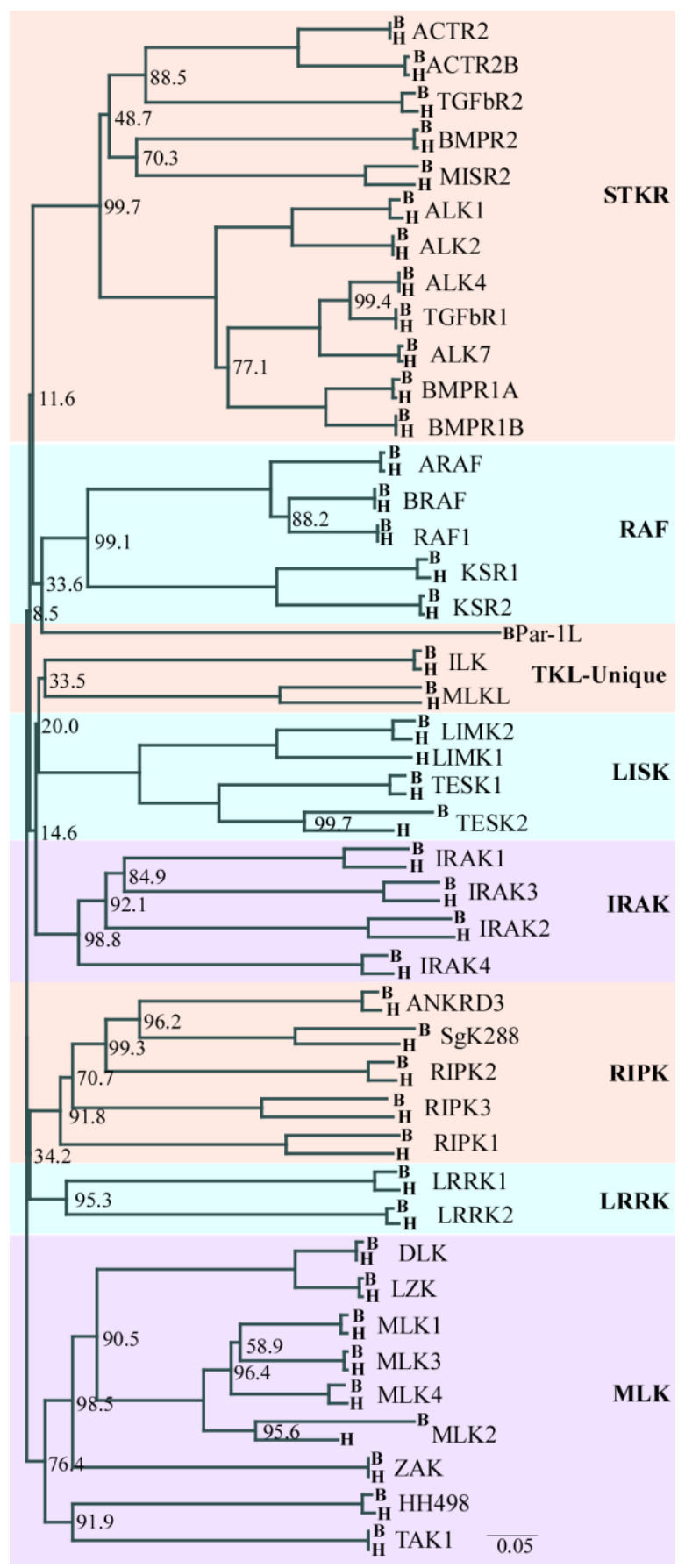

